*The version of Supporting Information published October 14, 2006, contained many errors, primarily in the nomenclature and spectral data. The revised Supporting Information was published January 18, 2007.*

\title{
Total Synthesis and Stereochemistry of the Antitumor Antibiotic, PD 113,271
}

Toshifumi Takeuchi, ${ }^{\dagger}$ Kouji Kuramochi, $^{\dagger}$ Susumu Kobayashi, ${ }^{\ddagger}$ and Fumio Sugawara $^{*, \dagger}$

Department of Applied Biological Science, Tokyo University of Science (RIKADAI), 2641 Yamazaki, Noda-shi, Chiba 278-8510 (Japan) and Faculty of Pharmaceutical Sciences, Tokyo University of Science (RIKADAI), 2641 Yamazaki, Noda-shi, Chiba 278-8510 (Japan)

sugawara@rs.noda.tus.ac.jp<smiles>C[C@@](O)(/C=C/C1OC(=O)C=CC1O)[C@@H](C[C@H](O)/C=C\C=C/C=C/CO)OP(=O)([O-])O[NH3+]</smiles>

PD 113,271 
General: ${ }^{1} \mathrm{H}-,{ }^{13} \mathrm{C}-$ and ${ }^{31} \mathrm{P}-\mathrm{NMR}$ were recorded on a BRUKER DXR400 or DRX600. Chemical shifts were reported in $\delta$, parts per million (ppm), relative to TMS as an internal standard or calibrated using residual undeuterated solvent as an internal reference. IR spectra were recorded on a JASCO FT/IR-410 spectrometer. Mass spectra were obtained on API QSTAR Pulsar i spectrometer. Optical rotations were measured on a JASCO P-1030 digital polarimeter. Melting points were determined with Yanaco MP-3S melting point apparatus. Column chromatography was carried out on Fuji Silisia PSQ100B. C ${ }_{18}$-reverse phase silica gel column chromatography was carried out on Fuji Silisia Chromatorex ODS (100-200 mesh). Analytical thin-layer chromatography (TLC) was performed on precoated Merck silica gel $60 \quad \mathrm{~F}_{254}$ plates, and compounds were visualized by UV illumination $(254 \mathrm{~nm})$ or heating $150^{\circ} \mathrm{C}$ after spraying phosphomolylbdic acid in ethanol. THF was distilled from sodium/benzophenone. $\mathrm{CH}_{2} \mathrm{Cl}_{2}$ was distilled from $\mathrm{P}_{2} \mathrm{O}_{5}$. HMPA and diisopropylamine were distilled from $\mathrm{CaH}_{2}$. All other solvent and reagents were obtained from commercial sources and used without further purification. Organic extracts were dried over $\mathrm{Na}_{2} \mathrm{SO}_{4}$, filtered, concentrated using a rotary evaporator. Involatile oils and solids were vacuum dried.

Methyl (2Z,4S,5S)-5,6-Isopropylidenedioxy-4-(4'-methoxybenzyloxy)-2-hexenoate (11)

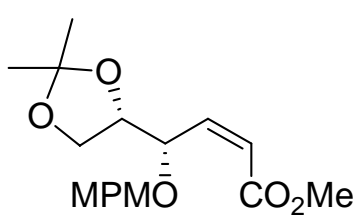

To a stirred solution of DMSO $(7.8 \mathrm{~mL}, 110 \mathrm{mmol})$ in $\mathrm{CH}_{2} \mathrm{Cl}_{2}(300 \mathrm{~mL})$ was added $(\mathrm{COCl})_{2}$ $\left(4.8 \mathrm{~mL}, 55 \mathrm{mmol}\right.$ ) at $-78^{\circ} \mathrm{C}$. The mixture was stirred for $15 \mathrm{~min}$ at the same temperature and a solution of $9^{1}(10.3 \mathrm{~g}, 36.5 \mathrm{mmol})$ in $\mathrm{CH}_{2} \mathrm{Cl}_{2}(46 \mathrm{~mL})$ was added dropwise at the same temperature. After $2.5 \mathrm{~h}$ at the same temperature, $\mathrm{Et}_{3} \mathrm{~N}(20.4 \mathrm{~mL}, 146 \mathrm{mmol})$ was added. After additional $30 \mathrm{~min}$, the reaction was quenched by the addition of phosphate buffer ( $\mathrm{pH} 7$ ), the reaction mixture was extracted with EtOAc. The combined organic layers were washed with $\mathrm{H}_{2} \mathrm{O}$ and saturated $\mathrm{NaCl}$ solution, dried over $\mathrm{Na}_{2} \mathrm{SO}_{4}$, and concentrated to provide the corresponding aldehyde as a pale yellow oil which was carried on directly to the next step without further purification.

To a stirred solution of $(\mathrm{PhO}){ }_{2} \mathrm{P}(\mathrm{O}) \mathrm{CH}_{2} \mathrm{CO}_{2} \mathrm{Me}(14.5 \mathrm{~g}, 47.4 \mathrm{mmol})$ in $\mathrm{THF}(370 \mathrm{~mL})$ were added $\mathrm{NaI}(5.47 \mathrm{~g}, 36.5 \mathrm{mmol})$ and $\mathrm{NaH}(60 \%)(1.75 \mathrm{~g}, 43.8 \mathrm{mmol})$ at $-78{ }^{\circ} \mathrm{C}$. The mixture was stirred for $15 \mathrm{~min}$ at $0{ }^{\circ} \mathrm{C}$, cooled to $-78{ }^{\circ} \mathrm{C}$. A solution of the crude aldehyde above in THF (36 mL) was stirred for $30 \mathrm{~min}$ at the same temperature. After addition of saturated $\mathrm{NH}_{4} \mathrm{Cl}$ solution, the reaction mixture was extracted with EtOAc. The combined organic layers were washed with $\mathrm{H}_{2} \mathrm{O}$ and saturated $\mathrm{NaCl}$ solution, dried over 
$\mathrm{Na}_{2} \mathrm{SO}_{4}$, and concentrated under reduced pressure. The resultant residue was purified by silica gel column chromatography (hexane/EtOAc = 9/1 to 4/1) to afford 11 (11.4 g, 93\%) as a colorless oil. $[\alpha]_{\mathrm{D}}{ }^{24}+5.5$ ( $\left.\mathrm{C} 0.93, \mathrm{CHCl}_{3}\right)$. IR $v_{\max }$ (neat): 2987, 1721, 1514, 1249, 1071, $826 \mathrm{~cm}^{-1} .{ }^{1} \mathrm{H}-\mathrm{NMR}\left(600 \mathrm{MHz}, \mathrm{CDCl}_{3}\right) \delta: 7.25(2 \mathrm{H}, d, J=8.6 \mathrm{~Hz}), 6.85$ $(2 \mathrm{H}, \mathrm{d}, J=8.6 \mathrm{~Hz}), 6.18(1 \mathrm{H}, \mathrm{dd}, J=11.8,9.2 \mathrm{~Hz}), 6.00(1 \mathrm{H}, \mathrm{dd}, J=11.8,1.0$ $\mathrm{Hz}), 5.14(1 \mathrm{H}, \mathrm{ddd}, J=9.2,5.3,1.0 \mathrm{~Hz}), 4.55(1 \mathrm{H}, \mathrm{d}, J=11.6 \mathrm{~Hz}), 4.43(1 \mathrm{H}, \mathrm{d}$, $J=11.6 \mathrm{~Hz}), 4.23(1 \mathrm{H}, t d, J=6.7,5.3 \mathrm{~Hz}), 3.94(1 \mathrm{H}, \mathrm{dd}, J=8.3,6.7 \mathrm{~Hz}), 3.89$ $(1 \mathrm{H}, \mathrm{dd}, J=8.3,6.7 \mathrm{~Hz}), 3.79(3 \mathrm{H}, \mathrm{s}), 3.71(3 \mathrm{H}, \mathrm{s}), 1.42(3 \mathrm{H}, \mathrm{s}), 1.35(3 \mathrm{H}, \mathrm{s})$. ${ }^{13} \mathrm{C}-\mathrm{NMR}\left(100 \mathrm{MHz}, \mathrm{CDCl}_{3}\right) \delta_{\mathrm{C}}: 166.1,159.1,146.3,130.2,129.4(2 \mathrm{C}), 122.8,113.6(2 \mathrm{C})$, 109.7, 77.6, 74.1, 71.2, 65.3, 55.2, 51.4, 26.2, 25.5. HR ESIMS m/z 359.1465 $\left(\mathrm{M}+\mathrm{Na}^{+}\right)(\mathrm{calcd}$ for 359.1465$)$.

$(2 z, 4 S, 5 S)-5,6$-Isopropylidenedioxy-4-(4'-methoxybenzyloxy) -2 -hexen-1-ol

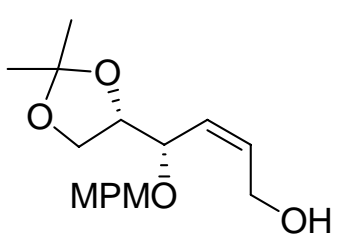

To a stirred solution of $11(2.00 \mathrm{~g}, 5.71 \mathrm{mmnol})$ in THF (5 mL) was added DIBAL (0.93 $\mathrm{M}$ in hexane, $15.3 \mathrm{~mL}, 14.2 \mathrm{mmol})$ at $0{ }^{\circ} \mathrm{C}$. The mixture was stirred for $30 \mathrm{~min}$ at the same temperature. After the addition of $\mathrm{H}_{2} \mathrm{O}$, the reaction mixture was poured into Erlenmeyer flask. After the addition of $\mathrm{Et}_{2} \mathrm{O}$ and Celite, the reaction mixture was stirred drastically for $1 \mathrm{~h}$ at room temperature, filtered through a pad of Celite. The filtrate was concentrated under reduced pressure. The resultant residue was purified by silica gel column chromatography (hexane/EtOAc $=4 / 1$ to $1 / 1$ ) to afford the alcohol $(1.73 \mathrm{~g}, 98 \%)$ as a colorless oil. $[\alpha]_{\mathrm{D}}{ }^{24}+27.0\left(\mathrm{C} 0.57, \mathrm{CHCl}_{3}\right)$. IR $v_{\max }$ (neat): $3444,2986,1613,1514,1372,1249,1070,849 \mathrm{~cm}^{-1} \cdot{ }^{1} \mathrm{H}-\mathrm{NMR}\left(600 \mathrm{MHz}, \mathrm{CDCl}_{3}\right) \delta: 7.25$ $(2 \mathrm{H}, \mathrm{d}, J=8.5 \mathrm{~Hz}), 6.87(2 \mathrm{H}, \mathrm{d}, J=8.5 \mathrm{~Hz}), 6.03(1 \mathrm{H}, \mathrm{dt}, J=11.1,6.3 \mathrm{~Hz}), 5.51$ $(1 \mathrm{H}, \mathrm{dd}, J=11.1,10.0 \mathrm{~Hz}), 4.60(1 \mathrm{H}, \mathrm{d}, J=11.7 \mathrm{~Hz}), 4.38(1 \mathrm{H}, \mathrm{d}, J=11.7 \mathrm{~Hz})$, $4.36(1 \mathrm{H}, \mathrm{dd}, J=10.0,6.5 \mathrm{~Hz}), 4.26(1 \mathrm{H}, \mathrm{q}, J=6.5 \mathrm{~Hz}), 4.09(2 \mathrm{H}, \mathrm{d}, J=6.3 \mathrm{~Hz})$, $3.98(1 \mathrm{H}, \mathrm{dd}, J=8.7,6.5 \mathrm{~Hz}), 3.78-3.82(1 \mathrm{H}, \mathrm{m}), 3.80(3 \mathrm{H}, \mathrm{s}), 2.18(1 \mathrm{H}, \mathrm{br} \mathrm{s}$, $\mathrm{OH}), 1.39(3 \mathrm{H}, \mathrm{s}), 1.34(3 \mathrm{H}, \mathrm{s}) \cdot{ }^{13} \mathrm{C}-\mathrm{NMR}\left(100 \mathrm{MHz}, \mathrm{CDCl}_{3}\right) \delta_{\mathrm{C}}: 159.3,134.8,130.0$, $129.4(2 \mathrm{C}), 128.8,113.8(2 \mathrm{C}), 109.6,76.9,73.9,70.3,65.3,58.7,55.3,26.3,25.1$. HR ESIMS m/z $331.1532\left(\mathrm{M}+\mathrm{Na}^{+}\right)$(calcd for 331.1515$)$.

$(5 S, 6 S)$-2 -Ethoxy-6-hydroxymethyl-5- (4'-methoxybenzyloxy) -5, 6-dihydro-2H-pyran (12)<smiles>CCOC1C=C[C@H](OCC)[C@H](CO)O1</smiles> 
To a stirred solution of the alcohol $\left(4.89 \mathrm{~g}, 15.8 \mathrm{mmol}\right.$ ) in $\mathrm{CH}_{2} \mathrm{Cl}_{2}$ (150 $\mathrm{mL}$ ) was added $\mathrm{MnO}_{2}(13.7 \mathrm{~g}, 158 \mathrm{mmol})$ at room temperature. After stirred overnight, the reaction mixture was filtered through a pad of Celite. The filtrate was concentrated under reduced pressure. The resultant aldehyde was carried on directly to the next step without further purification.

To a stirred solution of the crude aldehyde from above in EtOH (150 mL) was added CSA $(367 \mathrm{mg}, 1.58 \mathrm{mmol}$ ) at room temperature. The mixture was stirred for $12 \mathrm{~h}$ at the same temperature. After the addition of saturated $\mathrm{NaHCO}_{3}$ solution, the reaction mixture was extracted with EtOAc. The combined organic layers were washed with $\mathrm{H}_{2} \mathrm{O}$ and saturated $\mathrm{NaCl}$ solution, dried over $\mathrm{Na}_{2} \mathrm{SO}_{4}$, and concentrated under reduced pressure. The resultant residue was purified by silica gel column chromatography (hexane/EtOAC $=4 / 1$ to $2 / 1)$ to afford $12(3.22 \mathrm{~g}, 69 \%)$ as a white amorphous solid. $[\alpha]_{\mathrm{D}}^{24}+138.4$ $\left(\mathrm{C} 0.56, \mathrm{CHCl}_{3}\right)$. IR $v_{\max }(\mathrm{KBr}): 3293,2973,1615,1251,1067,891 \mathrm{~cm}^{-1} \cdot{ }^{1} \mathrm{H}-\mathrm{NMR}(600 \mathrm{MHz}$, $\left.\mathrm{CDCl}_{3}\right) \delta: 7.25(2 \mathrm{H}, \mathrm{d}, J=8.6 \mathrm{~Hz}), 6.88(2 \mathrm{H}, \mathrm{d}, J=8.6 \mathrm{~Hz}), 6.16(1 \mathrm{H}, \mathrm{ddd}, J=10.1$, $5.1,0.7 \mathrm{~Hz}), 6.03(1 \mathrm{H}, \mathrm{dd}, J=10.1,3.2 \mathrm{~Hz}), 5.12(1 \mathrm{H}, \mathrm{dd}, J=3.2,0.7 \mathrm{~Hz}), 4.62$ $(1 \mathrm{H}, \mathrm{d}, J=11.5 \mathrm{~Hz}), 4.46(1 \mathrm{H}, \mathrm{d}, J=11.5 \mathrm{~Hz}), 4.09(1 \mathrm{H}, \mathrm{ddd}, J=6.1,4.7,2.9$ $\mathrm{Hz}), 3.97(1 \mathrm{H}, \mathrm{ddd}, J=11.7,6.1,3.4 \mathrm{~Hz}), 3.85(1 \mathrm{H}, \mathrm{dq}, J=9.7,7.1 \mathrm{~Hz}), 3.81$ $(3 \mathrm{H}, \mathrm{s}), 3.80(1 \mathrm{H}, \mathrm{ddd}, J=11.7,9.4,4.7 \mathrm{~Hz}), 3.73(1 \mathrm{H}, \mathrm{dd}, J=5.1,2.9 \mathrm{~Hz}), 3.56$ $(1 \mathrm{H}, \mathrm{dq}, J=9.7,7.1 \mathrm{~Hz}), 2.23(1 \mathrm{H}$, br $\mathrm{dd}, J=9.4,3.4 \mathrm{~Hz}, \mathrm{OH}), 1.23(3 \mathrm{H}, t, J$ $=7.1 \mathrm{~Hz}) \cdot{ }^{13} \mathrm{C}-\mathrm{NMR}\left(100 \mathrm{MHz}, \mathrm{CDCl}_{3}\right) \delta_{\mathrm{C}}: 159.4,130.0,129.5(2 \mathrm{C}), 129.5,126.3,113.9$ (2C), 93.9, 70.4, 70.1, 67.8, 63.7, 62.9, 55.3, 15.3. HR ESIMS m/z 317.1361 $\left(\mathrm{M}+\mathrm{Na}^{+}\right)(\mathrm{calcd}$ for 317.1364$)$.

$(5 S, 6 S)$-2-Ethoxy-6-formyl-5- (4'-methoxybenzyloxy) -5,6-dihydro-2H-pyran (7)

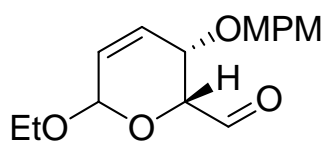

To a stirred solution of DMSO $(5.0 \mathrm{~mL}, 70 \mathrm{mmol})$ in $\mathrm{CH}_{2} \mathrm{Cl}_{2}(230 \mathrm{~mL})$ was added $(\mathrm{COCl})_{2}$ $(3.0 \mathrm{~mL}, 34 \mathrm{mmol})$ at $-78^{\circ} \mathrm{C}$. The mixture was stirred for $15 \mathrm{~min}$ at the same temperature and a solution of $12(6.83 \mathrm{~g}, 23.2 \mathrm{mmol})$ in $\mathrm{CH}_{2} \mathrm{Cl}_{2}$ (30 mL) was added dropwise at the same temperature. After $3.5 \mathrm{~h}$ at the same temperature, $\mathrm{Et}_{3} \mathrm{~N}$ (13 $\mathrm{mL}, 92.5 \mathrm{mmol}$ ) was added. After additional $10 \mathrm{~min}$, the mixture was allowed to warm to room temperature and the reaction was quenched by the addition of phosphate buffer ( $\mathrm{pH} 7$ ). The reaction mixture was extracted with $\mathrm{CH}_{2} \mathrm{Cl}_{2}$. The combined organic layers were washed with $\mathrm{H}_{2} \mathrm{O}$ and saturated $\mathrm{NaCl}$ solution, dried over $\mathrm{Na}_{2} \mathrm{SO}_{4}$, and concentrated under reduced pressure. The resultant residue was purified by silica gel column chromatography (hexane/EtOAC $=4 / 1$ to $2 / 1$ ) afford $7(6.56 \mathrm{~g}, 97 \%)$ as a pale yellow oil. $[\alpha]_{\mathrm{D}}^{24}+165.9\left(\mathrm{C} 1.0, \mathrm{CHCl}_{3}\right)$. IR $v_{\max }$ (neat) : 3460, 2975, 1740, 1515, 1250, 1051, $822 \mathrm{~cm}^{-1} .{ }^{1} \mathrm{H}-\mathrm{NMR}\left(600 \mathrm{MHz}, \mathrm{CDCl}_{3}\right)$ 
$\delta: 9.73(1 \mathrm{H}, \mathrm{s}), 7.20(2 \mathrm{H}, \mathrm{d}, J=8.5 \mathrm{~Hz}), 6.86(2 \mathrm{H}, \mathrm{d}, J=8.5 \mathrm{~Hz}), 6.07$ (1H, $\mathrm{dd}$, $J=10.2,5.0 \mathrm{~Hz}), 6.01(1 \mathrm{H}, \mathrm{dd}, J=10.2,2.9 \mathrm{~Hz}), 5.21(1 \mathrm{H}, \mathrm{d}, J=2.9 \mathrm{~Hz}), 4.50$ $(1 \mathrm{H}, \mathrm{d}, J=11.4 \mathrm{~Hz}), 4.48(1 \mathrm{H}, \mathrm{d}, J=3.3 \mathrm{~Hz}), 4.46(1 \mathrm{H}, \mathrm{d}, J=11.4 \mathrm{~Hz}), 4.10(1 \mathrm{H}$, $\mathrm{dd}, J=5.0,3.3 \mathrm{~Hz}), 3.88(1 \mathrm{H}, \mathrm{dq}, J=9.7,7.1 \mathrm{~Hz}), 3.80(3 \mathrm{H}, \mathrm{s}), 3.61$ (1H, dq, $J=9.7,7.1 \mathrm{~Hz}), 1.22(3 \mathrm{H}, t, J=7.1 \mathrm{~Hz}) \cdot{ }^{13} \mathrm{C}-\mathrm{NMR}\left(100 \mathrm{MHz}, \mathrm{CDCl}_{3}\right) \delta_{\mathrm{C}}: 200.3,159.3$ ， $129.8,129.7,129.6(2 \mathrm{C}), 125.7,113.7(2 \mathrm{C}), 93.9,75.5,70.9,67.2,64.2,55.2$ ， 15.1. HR ESIMS m/z 315.1218 (M+Na+) (calcd for 315.12022).

$\left(2 R, 4 R, 1^{\prime} R\right)-2$ - tert-Butyldimethylsiloxy-4-(1', $2^{\prime}-$ isopropylidenedioxylethyl) $-\mathrm{Y}-\mathrm{bu}$ tyrolactone

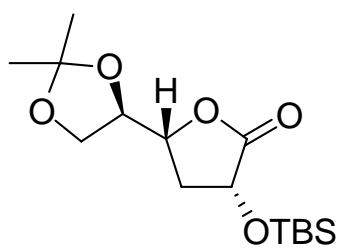

To a stirred solution of $10^{2}(58.8 \mathrm{~g}, 291 \mathrm{mmol})$ in DMF $(300 \mathrm{~mL})$ were added imidazole (39.6 $\mathrm{g}, 582 \mathrm{mmol})$ and $\mathrm{TBSCl}(65.8 \mathrm{~g}, 455 \mathrm{mmol})$ at room temperature. The resultant mixture was stirred at the same temperature for $2 \mathrm{~h}$. After the addition of $\mathrm{NaHCO}_{3}$ solution, the reaction mixture was extracted with EtOAc. The combined organic layers were washed with $\mathrm{H}_{2} \mathrm{O}$ and saturated $\mathrm{NaCl}$ solution, dried over $\mathrm{Na}_{2} \mathrm{SO}_{4}$, and concentrated under reduced pressure. The resultant residue was purified by silica gel column chromatography (hexane $/$ EtOAC $=9 / 1$ to $4 / 1$ ) to afford the lactone $(91.8 \mathrm{~g}, 100 \%)$ as a colorless oil. $[\alpha]_{D}{ }^{24}+5.0\left(\mathrm{C} 0.92, \mathrm{CHCl}_{3}\right)$. IR $v_{\max }$ (neat): 2954, 2932, 1792, 1255, $1155,840 \mathrm{~cm}^{-1} \cdot{ }^{1} \mathrm{H}-\mathrm{NMR}\left(600 \mathrm{MHz}, \mathrm{CDCl}_{3}\right) \delta: 4.50(1 \mathrm{H}, \mathrm{dd}, J=10.4,8.3 \mathrm{~Hz}), 4.34$ (1H, $d t, J=10.4,5.4 \mathrm{~Hz}), 4.25(1 \mathrm{H}, \mathrm{td}, J=6.4,5.4 \mathrm{~Hz}), 4.09(1 \mathrm{H}, \mathrm{dd}, J=8.7,6.4$ $\mathrm{Hz}), 3.87(1 \mathrm{H}, \mathrm{dd}, J=8.7,6.4 \mathrm{~Hz}), 2.48(1 \mathrm{H}, \mathrm{ddd}, J=12.5,8.3,5.4 \mathrm{~Hz}), 2.08$ $(1 \mathrm{H}, \mathrm{dt}, J=12.5,10.4 \mathrm{~Hz}), 1.44(3 \mathrm{H}, \mathrm{s}), 1.38(3 \mathrm{H}, \mathrm{s}), 0.92(9 \mathrm{H}, \mathrm{s}), 0.18(3 \mathrm{H}$, $\mathrm{s}), 0.15(3 \mathrm{H}, \mathrm{s}) \cdot{ }^{13} \mathrm{C}-\mathrm{NMR}\left(100 \mathrm{MHz}, \mathrm{CDCl}_{3}\right) \delta_{\mathrm{C}}: 174.8,110.3,76.2,75.1,68.8,64.8$, $33.6,26.3,25.6(3 \mathrm{C}), 25.2,18.2,-4.7,-5.3$. HR ESIMS m/z 339.1614(M+Na $\left.{ }^{+}\right)(\mathrm{calcd}$ for 339.1598$)$.

Dimethyl

(3'R, 5'R, 1' R) -1-[3' - (tert-Butyldimethylsiloxy) -2' -hydroxy-4' - (1' ', 2' ' -isopropylidenedio xyethyl) - tetrahydrofuran-2'-yl]-methylphosphonate (13)

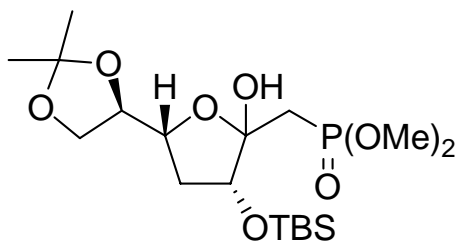

To a stirred solution of dimethyl methylphosphonate (9.0 mL, $83.1 \mathrm{mmol})$ in THF (300 
$\mathrm{mL})$ was added $n$-BuLi (2.44 $\mathrm{M}$ in hexane, $31.7 \mathrm{~mL}, 77.3 \mathrm{mmol})$ at $-78{ }^{\circ} \mathrm{C}$. The mixture was stirred for $15 \mathrm{~min}$ at the same temperature and a solution of the lactone (8.72 g, $27.6 \mathrm{mmol}$ ) in THF (40 mL) was added dropwise at the same temperature. After stirred for $15 \mathrm{~min}$ at $0{ }^{\circ} \mathrm{C}$, saturated $\mathrm{NH}_{4} \mathrm{Cl}$ solution was added and the reaction mixture was extracted with EtOAc. The combined organic layers were washed with $\mathrm{H}_{2} \mathrm{O}$ and saturated $\mathrm{NaCl}$ solution, dried over $\mathrm{Na}_{2} \mathrm{SO}_{4}$, and concentrated under reduced pressure. The resultant residue was purified by silica gel column chromatography (hexane/EtOAC $=4 / 1$ to $1 / 1$ ) to afford $13\left(10.7 \mathrm{~g}, 88 \%\right.$ ) as a colorless oil. $[\alpha]_{\mathrm{D}}^{24}-11.2$ (c 0.45, $\left.\mathrm{CHCl}_{3}\right)$. IR $v_{\max }$ (neat): 3386, 2955, 1463, 1371, 1039, $838 \mathrm{~cm}^{-1} \cdot{ }^{1} \mathrm{H}-\mathrm{NMR}\left(600 \mathrm{MHz}, \mathrm{CDCl}_{3}\right)$ $\delta: 5.36(1 \mathrm{H}, \mathrm{br} \mathrm{s}, \mathrm{OH}), 4.28(1 \mathrm{H}, \mathrm{dt}, J=8.4,6.4 \mathrm{~Hz}), 4.23(1 \mathrm{H}, \mathrm{q}, J=6.4 \mathrm{~Hz})$, $4.10(1 \mathrm{H}, \mathrm{br} d d, J=5.8,1.0 \mathrm{~Hz}), 3.97(1 \mathrm{H}, \mathrm{dd}, J=8.3,6.4 \mathrm{~Hz}), 3.90(3 \mathrm{H}, d, J$ $=11.1 \mathrm{~Hz}), 3.82(1 \mathrm{H}, \mathrm{dd}, J=8.3,6.4 \mathrm{~Hz}), 3.74(3 \mathrm{H}, \mathrm{d}, J=11.1 \mathrm{~Hz}), 2.45(1 \mathrm{H}$, $\mathrm{ddd}, J=13.7,8.4,5.8 \mathrm{~Hz}), 2.42(1 \mathrm{H}, \mathrm{dd}, J=18.1,15.1 \mathrm{~Hz}), 2.10(1 \mathrm{H}, \mathrm{dd}, J=$ $18.1,15.1 \mathrm{~Hz}), 1.52(1 \mathrm{H}, \mathrm{ddt}, J=13.7,6.4,1.0 \mathrm{~Hz}), 1.41(3 \mathrm{H}, \mathrm{s}), 1.34(3 \mathrm{H}, \mathrm{s})$, $0.90(9 \mathrm{H}, \mathrm{s}), 0.08(6 \mathrm{H}, \mathrm{s}) \cdot{ }^{13} \mathrm{C}-\mathrm{NMR}\left(100 \mathrm{MHz}, \mathrm{CDCl}_{3}\right) \delta_{\mathrm{C}}: 109.6,105.4\left(\mathrm{~d}, J_{\mathrm{C}-\mathrm{P}}=8.1\right.$ $\mathrm{Hz}), 78.1,77.5,77.3\left(\mathrm{~d}, J_{\mathrm{C}-\mathrm{P}}=11.1 \mathrm{~Hz}\right), 65.6,53.9\left(\mathrm{~d}, J_{\mathrm{C}-\mathrm{P}}=5.7 \mathrm{~Hz}\right), 51.6\left(\mathrm{~d}, J_{\mathrm{C}-\mathrm{P}}\right.$ $=6.8 \mathrm{~Hz}), 35.5,31.0\left(\mathrm{~d}, J_{\mathrm{C}-\mathrm{P}}=136.9 \mathrm{~Hz}\right), 26.6,25.8(3 \mathrm{C}), 25.2,18.0,-4.6,-5.0$. HR ESIMS m/z $463.1890\left(\mathrm{M}^{\mathrm{N}} \mathrm{Na}^{+}\right)(\mathrm{calcd}$ for 463.1887$)$.

\section{Dimethyl}

$(3 R, 5 R, 6 R)-3,5$-Bis (tert-butyldimethylsiloxy) -6, 7-isopropylidenedioxy-2-heptanon ylphosphonate (8)

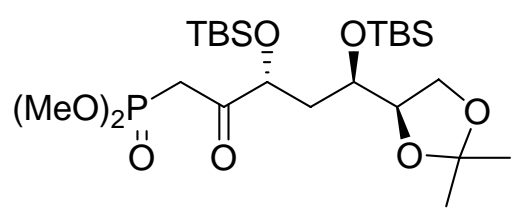

To a stirred solution of $13(11.0 \mathrm{~g}, 25 \mathrm{mmol})$ in THF (160 mL) was added KOt-Bu (8.42 g, $75 \mathrm{mmol}$ ) at $-78^{\circ} \mathrm{C}$. Then TBSCl (15.1 g, $\left.100 \mathrm{mmol}\right)$ was added to the mixture, and the mixture was stirred for $1 \mathrm{~h}$ at room temperature. After the addition of saturated $\mathrm{NH}_{4} \mathrm{Cl}$ solution, the reaction mixture was extracted with EtOAc. The combined organic layers were washed with $\mathrm{H}_{2} \mathrm{O}$ and saturated $\mathrm{NaCl}$ solution, dried over $\mathrm{Na}_{2} \mathrm{SO}_{4}$, and concentrated under reduced pressure. The resultant residue was purified by silica gel column chromatography (hexane/EtOAc $=4 / 1$ to $2 / 1$ ) to afford 8 (13.9 9, 100\%) as a colorless oil. [ $[\alpha]_{\mathrm{D}}^{24}-11.3\left(\mathrm{C} 0.51, \mathrm{CHCl}_{3}\right)$. IR $v_{\max }$ (neat): 2955, 1730, 1472, 1371, $1255,1035,838 \mathrm{~cm}^{-1} \cdot{ }^{1} \mathrm{H}-\mathrm{NMR}\left(600 \mathrm{MHz}, \mathrm{CDCl}_{3}\right) \delta: 4.40(1 \mathrm{H}, \mathrm{dd}, J=7.7,4.2 \mathrm{~Hz}), 4.05$ $(1 \mathrm{H}, \mathrm{ddd}, J=7.4,6.6,5.8 \mathrm{~Hz}), 3.94(1 \mathrm{H}, \mathrm{dd}, J=8.1,6.6 \mathrm{~Hz}), 3.84(1 \mathrm{H}, \mathrm{ddd}, J$ $=8.0,5.8,3.9 \mathrm{~Hz}), 3.80(3 \mathrm{H}, \mathrm{d}, 1.5 \mathrm{~Hz}), 3.78(3 \mathrm{H}, \mathrm{d}, J=1.4 \mathrm{~Hz}), 3.67(1 \mathrm{H}, \mathrm{dd}$, $J=8.1,7.4 \mathrm{~Hz}), 3.30(1 \mathrm{H}, \mathrm{dd}, J=21.4,14.8 \mathrm{~Hz}), 3.07(1 \mathrm{H}, \mathrm{dd}, J=22.3,14.8$ 
$\mathrm{Hz}), 1.76(1 \mathrm{H}, \mathrm{ddd}, J=13.9,8.0,4.2 \mathrm{~Hz}), 1.66(1 \mathrm{H}, \mathrm{ddd}, \mathrm{J}=13.9,7.7,3.9 \mathrm{~Hz})$, $1.40(3 \mathrm{H}, \mathrm{s}), 1.33(3 \mathrm{H}, \mathrm{s}), 0.92(9 \mathrm{H}, \mathrm{s}), 0.90(9 \mathrm{H}, \mathrm{s}), 0.11(3 \mathrm{H}, \mathrm{s}), 0.11(3 \mathrm{H}, \mathrm{s})$, $0.09(3 \mathrm{H}, \mathrm{s}), 0.08(3 \mathrm{H}, \mathrm{s}) \cdot{ }^{13} \mathrm{C}-\mathrm{NMR}\left(100 \mathrm{MHz}, \mathrm{CDCl}_{3}\right) \delta_{\mathrm{C}}: 200.3\left(\mathrm{~d}, \mathrm{~J}_{\mathrm{C}-\mathrm{P}}=6.9 \mathrm{~Hz}\right), 109.3$, $78.0,75.9\left(\mathrm{~d}, J_{\mathrm{C}-\mathrm{P}}=3.4 \mathrm{~Hz}\right), 70.2,65.6,52.9\left(2 \mathrm{C}, \mathrm{d}, J_{\mathrm{C}-\mathrm{P}}=6.4 \mathrm{~Hz}\right), 38.2,35.6(\mathrm{~d}$, $\left.J_{\mathrm{C}-\mathrm{P}}=133.8 \mathrm{~Hz}\right), 26.4,25.9(3 \mathrm{C}), 25.8(3 \mathrm{C}), 25.3,18.2,18.1,-4.0,-4.5(2 \mathrm{C}),-4.9$. HR ESIMS m/z 577.2754 (M+Na ${ }^{+}$) (calcd for 577.2752).

$\left(5 S, 6 S, 1^{\prime} E, 4^{\prime} R, 6^{\prime} R, 7^{\prime} R\right)-6-\left[4^{\prime}, 6^{\prime}\right.$-Bis (tert-butyldimethylsiloxy) -7', 8'-isopropyli denedioxy-3' -oxo-1' -octenyl] -2-ethoxy-5,6-dihydro-5- (4' ' -methoxybenzyloxy) - 2H-p yran (14)

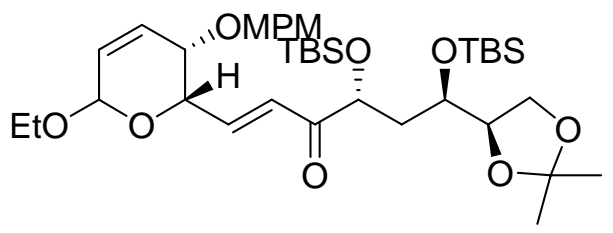

To a stirred solution of 7 (6.0 g, $20.5 \mathrm{mmol})$ and 8 (8.1 $\mathrm{g}, 14.6 \mathrm{mmol})$ in toluene $(200 \mathrm{~mL})$ was added $\mathrm{KOt}-\mathrm{Bu}(1.8 \mathrm{~g}, 16 \mathrm{mmol})$ at $0{ }^{\circ} \mathrm{C}$ and the mixture was stirred for $30 \mathrm{~min}$ at the same temperature. After the addition of saturated $\mathrm{NH}_{4} \mathrm{Cl} \mathrm{solution}$ the reaction mixture was extracted with EtOAc. The combined organic layers were washed with $\mathrm{H}_{2} \mathrm{O}$ and saturated $\mathrm{NaCl}$ solution, dried over $\mathrm{Na}_{2} \mathrm{SO}_{4}$, and concentrated under reduced pressure. The resultant residue was purified by silica gel column chromatography (hexane $/$ EtoAc $=9 / 1$ to $4 / 1)$ to afford $14\left(9.8 \mathrm{~g}, 93 \%\right.$ ) as a colorless oil. $[\alpha]_{\mathrm{D}}^{24}+141.2$ $\left(\mathrm{C} 0.25, \mathrm{CHCl}_{3}\right)$. IR $v_{\max }$ (neat): 2930, 1741, 1515, 1372, 1250, $837 \mathrm{~cm}^{-1} \cdot{ }^{1} \mathrm{H}-\mathrm{NMR}(600$ $\left.\mathrm{MHz}, \mathrm{CDCl}_{3}\right) \delta: 7.18(2 \mathrm{H}, \mathrm{d}, J=8.5 \mathrm{~Hz}), 7.11(1 \mathrm{H}, \mathrm{dd}, J=15.6,3.6 \mathrm{~Hz}), 6.84(2 \mathrm{H}$, $\mathrm{d}, J=8.5 \mathrm{~Hz}), 6.78(1 \mathrm{H}, \mathrm{dd}, J=15.6,2.0 \mathrm{~Hz}), 6.10(1 \mathrm{H}, \mathrm{dd}, \mathrm{J}=10.1,5.1 \mathrm{~Hz})$, $6.02(1 \mathrm{H}, \mathrm{dd}, J=10.1,2.9 \mathrm{~Hz}), 5.15(1 \mathrm{H}, \mathrm{d}, J=2.9 \mathrm{~Hz}), 4.73(1 \mathrm{H}, \mathrm{ddd}, \mathrm{J}=3.6$, $3.0,2.0 \mathrm{~Hz}), 4.51(1 \mathrm{H}, \mathrm{dd}, J=8.7,3.0 \mathrm{~Hz}), 4.47(1 \mathrm{H}, \mathrm{d}, J=11.8 \mathrm{~Hz}), 4.44(1 \mathrm{H}$, $\mathrm{d}, J=11.8 \mathrm{~Hz}), 4.01(1 \mathrm{H}, \mathrm{q}, J=6.7 \mathrm{~Hz}), 3.90(1 \mathrm{H}, \mathrm{dd}, J=7.8,6.7 \mathrm{~Hz}), 3.87(1 \mathrm{H}$, $\mathrm{ddd}, J=8.7,6.7,2.4 \mathrm{~Hz}), 3.81(1 \mathrm{H}, \mathrm{dd}, \mathrm{J}=9.7,7.1 \mathrm{~Hz}), 3.79(3 \mathrm{H}, \mathrm{s}), 3.77(1 \mathrm{H}$, $\mathrm{dd}, J=5.1,3.0 \mathrm{~Hz}), 3.61(1 \mathrm{H}, \mathrm{dd}, J=7.8,6.7 \mathrm{~Hz}), 3.57(1 \mathrm{H}, \mathrm{dd}, \mathrm{J}=9.7,7.1$ $\mathrm{Hz}), 1.75(1 \mathrm{H}, \mathrm{ddd}, J=13.8,8.7,3.0 \mathrm{~Hz}), 1.53(1 \mathrm{H}, \mathrm{ddd}, \mathrm{J}=13.8,8.7,2.4 \mathrm{~Hz})$, $1.40(3 \mathrm{H}, \mathrm{s}), 1.34(3 \mathrm{H}, \mathrm{s}), 1.22(3 \mathrm{H}, \mathrm{t}, \mathrm{J}=7.1 \mathrm{~Hz}), 0.92(9 \mathrm{H}, \mathrm{s}), 0.90(9 \mathrm{H}, \mathrm{s})$, $\left.0.12(6 \mathrm{H}, \mathrm{s}), 0.09(3 \mathrm{H}, \mathrm{s}), 0.05(3 \mathrm{H}, \mathrm{s}) \cdot{ }^{13} \mathrm{C}-\mathrm{NMR}(100 \mathrm{MHz}, \mathrm{CDCl})_{3}\right) \delta_{\mathrm{C}}: 199.9,160.6$, $143.6,130.1,129.6,129.3(2 \mathrm{C}), 127.0,124.2,113.8$ (2C), 109.2, 94.1, 78.7, 75.1, $71.0,70.5,70.0,68.3,65.8,63.9,55.2,26.4,26.0(3 \mathrm{C}), 25.9(3 \mathrm{C}), 25.4,18.3$, $18.3,15.3,-3.8,-4.1,-4.7,-4.8$. HR ESIMS m/z $743.3985\left(\mathrm{M}+\mathrm{Na}^{+}\right.$) (calcd for 743.3981$)$.

$\left(5 S, 6 S, 1^{\prime} E, 3^{\prime} R, 4^{\prime} R, 6^{\prime} R, 7^{\prime} R\right)-6-\left[4^{\prime}, 6^{\prime}\right.$-Bis (tert-butyldimethylsiloxy) - 3'-hydroxy-7 ', 8' -isopropylidenedioxy-3' -methyl-1'-octenyl] -2-ethoxy-5,6-dihydro-5-(4' '-meth 
$\left(5 S, 6 S, 1^{\prime} E, 3^{\prime} S, 4^{\prime} R, 6^{\prime} R, 7^{\prime} R\right)-6-\left[4^{\prime}, 6^{\prime}-\mathrm{Bis}\right.$ (tert-butyldimethylsiloxy) -3' -hydroxy-7 ' , 8' -isopropylidenedioxy-3'-methyl-1' -octenyl] -2-ethoxy-5,6-dihydro-5- (4' '-meth oxybenzyloxy) - $2 H$-pyran (15b)

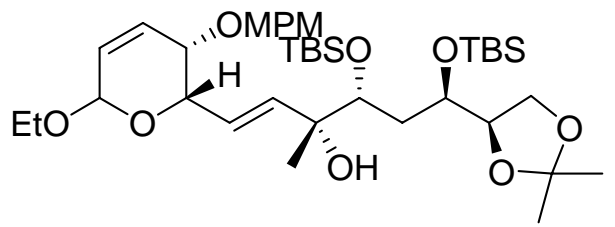

$\mathrm{CeCl}_{3} \cdot 7 \mathrm{H}_{2} \mathrm{O}(5.0 \mathrm{~g}, 20.3 \mathrm{mmol})$ was placed in a $200 \mathrm{~mL}$ flask and dried under vacuum at $80^{\circ} \mathrm{C}$ for $1 \mathrm{~h}$ and then at $140^{\circ} \mathrm{C}$ for $1 \mathrm{~h}$. After cooling to room temperature, anhydrous THF (50 mL) was added and the resulting slurry was stirred overnight. The slurry was cooled to $-78^{\circ} \mathrm{C}$ and MeLi ( $1.02 \mathrm{M}$ in Et $\mathrm{E}_{2}, 9.5 \mathrm{~mL}, 9.7 \mathrm{mmol}$ ) was added. The mixture was stirred for $5 \mathrm{~min}$ at $0{ }^{\circ} \mathrm{C}$, then cooled to $-78^{\circ} \mathrm{C}$. A solution 14 ( $1 \mathrm{~g}, 1.39 \mathrm{mmol}$ ) in THF (15 mL) was added to the mixture, and the mixture was stirred at $-78{ }^{\circ} \mathrm{C}$ for $10 \mathrm{~min}$. Then the mixture was allowed to warm to $0{ }^{\circ} \mathrm{C}$ and the reaction was quenched by the addition of $\mathrm{NH}_{4} \mathrm{Cl}$ solution, the reaction mixture was extracted with EtOAc. The combined organic layers were washed with $\mathrm{H}_{2} \mathrm{O}$ and saturated $\mathrm{NaCl}$ solution, dried over $\mathrm{Na}_{2} \mathrm{SO}_{4}$, and concentrated under reduced pressure. The resultant residue was purified by silica gel column chromatography (hexane/EtOAc = 4/1 to 3.25/1) afford $15 \mathrm{a}(767 \mathrm{mg}, 75 \%)$ and its (8S)-isomer (15b) (179 mg, 17\%) each as a colorless oil. 15a: $[\alpha]_{\mathrm{D}}^{24}+70.3\left(\mathrm{C} 0.37, \mathrm{CHCl}_{3}\right)$. IR $v_{\max }$ (neat) : 2955, 1514, 1251, 1092, $838 \mathrm{~cm}^{-1} .{ }^{1} \mathrm{H}-\mathrm{NMR}$ $\left(600 \mathrm{MHz}, \mathrm{CDCl}_{3}\right) \delta: 7.25(2 \mathrm{H}, \mathrm{d}, J=8.8 \mathrm{~Hz}), 6.85(2 \mathrm{H}, \mathrm{d}, J=8.8 \mathrm{~Hz}), 6.07(1 \mathrm{H}$, $\mathrm{dd}, J=10.1,5.2 \mathrm{~Hz}), 6.02(1 \mathrm{H}, \mathrm{dd}, J=15.7,4.7 \mathrm{~Hz}), 5.98(1 \mathrm{H}, \mathrm{dd}, J=10.1,2.9$ $\mathrm{Hz}), 5.97(1 \mathrm{H}, \mathrm{dd}, J=15.7,0.9 \mathrm{~Hz}), 5.11(1 \mathrm{H}, \mathrm{d}, J=2.9 \mathrm{~Hz}), 4.56$ (1H, br ddd, $J=4.7,2.8,0.9 \mathrm{~Hz}), 4.53(1 \mathrm{H}, \mathrm{d}, J=11.6 \mathrm{~Hz}), 4.50(1 \mathrm{H}, \mathrm{d}, J=11.6 \mathrm{~Hz}), 3.86-3.92$ $(2 \mathrm{H}, \mathrm{m}), 3.84(1 \mathrm{H}, \mathrm{d}, J=10.0,6.2,2.0 \mathrm{~Hz}), 3.81(1 \mathrm{H}, \mathrm{dd}, J=9.7,7.1 \mathrm{~Hz}), 3.79$ $(3 \mathrm{H}, \mathrm{s}), 3.78(1 \mathrm{H}, \mathrm{dd}, J=7.0,2.6 \mathrm{~Hz}), 3.66(1 \mathrm{H}, \mathrm{dd}, J=5.2,2.8 \mathrm{~Hz}), 3.57$ (1H, $t, J=6.8 \mathrm{~Hz}), 3.55(1 \mathrm{H}, \mathrm{dd}, J=9.7,7.1 \mathrm{~Hz}), 2.25(1 \mathrm{H}, \mathrm{br} \mathrm{s}, \mathrm{OH}), 1.70$ ( $1 \mathrm{H}, \mathrm{ddd}$, $J=14.2,10.0,2.6 \mathrm{~Hz}), 1.39(3 \mathrm{H}, \mathrm{s}), 1.32(3 \mathrm{H}, \mathrm{s}), 1.27(3 \mathrm{H}, \mathrm{s}), 1.26$ ( $1 \mathrm{H}$, ddd, $J=14.2,7.0,2.0 \mathrm{~Hz}), 1.21(3 \mathrm{H}, t, J=7.1 \mathrm{~Hz}), 0.90(9 \mathrm{H}, \mathrm{s}), 0.89(9 \mathrm{H}, \mathrm{s}), 0.13$ $(6 \mathrm{H}, \mathrm{s}), 0.12(3 \mathrm{H}, \mathrm{s}), 0.11(3 \mathrm{H}, \mathrm{s}) \cdot{ }^{13} \mathrm{C}-\mathrm{NMR}\left(100 \mathrm{MHz}, \mathrm{CDCl}_{3}\right) \delta_{\mathrm{C}}: 159.1,136.1,130.8$, $129.4,129.2(2 \mathrm{C}), 127.5,126.4,113.7(2 \mathrm{C}), 109.3,94.1,79.1,76.0,75.3,72.0$, $70.6,70.6,69.2,65.7,63.8,55.2,37.5,26.4,26.0(3 \mathrm{C}), 26.0(3 \mathrm{C}), 25.3,23.8$, $18.3,18.2,15.3,-3.6(2 \mathrm{C}),-4.0,-4.3$. HR ESIMS $\mathrm{m} / \mathrm{z} 759.4290\left(\mathrm{M}+\mathrm{Na}^{+}\right.$) (calcd for $759.4294)$.

15b: $[\alpha]_{\mathrm{D}}^{24}+70.3\left(\mathrm{C} 0.77, \mathrm{CHCl}_{3}\right) . \operatorname{IR~} v_{\max }$ (neat): 2930, 1613, 1514, 1251, 1095, 837 $\mathrm{cm}^{-1} .{ }^{1} \mathrm{H}-\mathrm{NMR}\left(600 \mathrm{MHz}, \mathrm{CDCl}_{3}\right) \delta: 7.26(2 \mathrm{H}, \mathrm{d}, J=8.6 \mathrm{~Hz}), 6.85(2 \mathrm{H}, \mathrm{d}, J=8.6 \mathrm{~Hz})$, 
$6.08(1 \mathrm{H}, \mathrm{dd}, J=10.1,5.1 \mathrm{~Hz}), 6.03(1 \mathrm{H}, \mathrm{dd}, J=15.8,6.4 \mathrm{~Hz}), 5.98(1 \mathrm{H}, \mathrm{dd}, J$ $=10.1,2.9 \mathrm{~Hz}), 5.94(1 \mathrm{H}, \mathrm{dd}, J=15.8 \mathrm{~Hz}), 5.09(1 \mathrm{H}, \mathrm{d}, J=2.9 \mathrm{~Hz}), 4.53(1 \mathrm{H}$, $d, J=11.6 \mathrm{~Hz}), 4.51(1 \mathrm{H}, \mathrm{dd}, J=11.6 \mathrm{~Hz}), 4.50(1 \mathrm{H}, \mathrm{dd}, J=6.4,2.7 \mathrm{~Hz}), 3.83-3.92$ $(2 \mathrm{H}, \mathrm{m}), 3.84(1 \mathrm{H}, \mathrm{d}, J=10.0,2.1 \mathrm{~Hz}), 3.79(3 \mathrm{H}, \mathrm{s}), 3.79(1 \mathrm{H}, \mathrm{dd}, J=9.7,7.1$ $\mathrm{Hz}), 3.77(1 \mathrm{H}, \mathrm{dd}, J=6.8,2.3 \mathrm{~Hz}), 3.61(1 \mathrm{H}, \mathrm{dd}, J=5.1,2.7 \mathrm{~Hz}), 3.57(1 \mathrm{H}, t$, $J=7.2 \mathrm{~Hz}), 3.55(1 \mathrm{H}, \mathrm{dd}, J=9.7,7.1 \mathrm{~Hz}), 2.39(1 \mathrm{H}, \mathrm{s}), 1.70(1 \mathrm{H}, \mathrm{ddd}, J=14.2$, $10.0,2.3 \mathrm{~Hz}), 1.39(3 \mathrm{H}, \mathrm{s}), 1.32(3 \mathrm{H}, \mathrm{s}), 1.29(1 \mathrm{H}, \mathrm{ddd}, J=14.2,6.8,2.1 \mathrm{~Hz})$, $1.26(3 \mathrm{H}, \mathrm{s}), 1.21(3 \mathrm{H}, \mathrm{t}, J=7.1 \mathrm{~Hz}), 0.90(9 \mathrm{H}, \mathrm{s}), 0.89(9 \mathrm{H}, \mathrm{s}), 0.14(3 \mathrm{H}, \mathrm{s})$, $0.13(3 \mathrm{H}, \mathrm{s}), 0.12(3 \mathrm{H}, \mathrm{s}), 0.11(3 \mathrm{H}, \mathrm{s}) \cdot{ }^{13} \mathrm{C}-\mathrm{NMR}\left(100 \mathrm{MHz}, \mathrm{CDCl}_{3}\right) \delta_{\mathrm{C}}: 159.1,136.3$, $130.7,129.4,129.3(2 \mathrm{C}), 127.6,126.1,113.7$ (2C), 109.3, 94.0, 79.1, 76.2, 75.0, $72.0,71.2,70.6,69.4,65.6,63.6,55.2,37.6,26.4,26.0(6 \mathrm{C}), 25.3,23.8,18.2$, $15.4,15.3,-3.4,-3.6,-4.1,-4.3$. HR ESIMS m/z $759.4316\left(\mathrm{M}+\mathrm{Na}^{+}\right)$(calcd for 759.4294$)$.

\section{Determination of $15 \mathrm{a}$ and $15 \mathrm{~b}$.}

The configurationes of $15 \mathrm{a}$ and $15 \mathrm{~b}$ was determined by NOESY experiments of the corresponding acetonide derivatives $\mathbf{s 1}$ and $\mathbf{S 2}$, respectively, in three steps (Supporting Information Scheme 1 ). The 2D 1H-1H NOESY experiment $\left(\mathrm{D}_{2} \mathrm{O}, 600 \mathrm{MHz}, \delta\right.$ ) in S1 showed correlations between $\mathrm{H}-7$ (5.86) and $\mathrm{H}-9(4.01-4.07)$ and between $\mathrm{C} 8-\mathrm{CH}_{3}$ (1.21) and H-10 (1.37-1.42, 1.50 - 1.55), establishing that the relationship of the 1,2-diol between C8 and C9 was syn. NOESY correlations between $H-7$ (5.79) and $\mathrm{H}-10(1.31-1.39,1.42-1.48)$ and between $\mathrm{C} 8-\mathrm{CH}_{3}(1.38)$ and $\mathrm{H}-9$ (4.08) of s2 were observed, indicating that the relationship of the 1,2-diol orientation between C8 and $\mathrm{C} 9$ could be assigned as anti.

\section{Supporting Information Scheme 1.}

$15 a$

1) TBAF, THF, $100 \%$

2) TBSOTf, 2,6-lutidine, $\mathrm{CH}_{2} \mathrm{Cl}_{2}, 61 \%$

3) $(\mathrm{MeO})_{2} \mathrm{CMe}_{2}, \mathrm{CSA}$, acetone

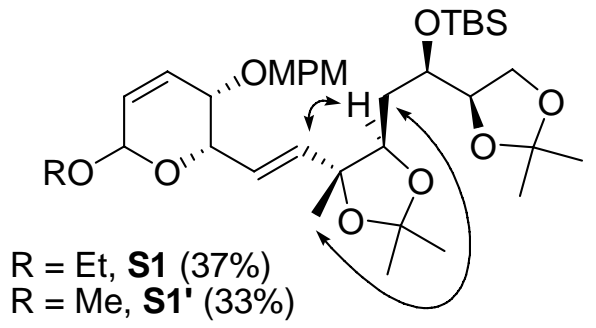

1) TBAF, THF, $42 \%$

2) TBSOTf, 2,6-lutidine, $\mathrm{CH}_{2} \mathrm{Cl}_{2}, 72 \%$

15b

3) $(\mathrm{MeO})_{2} \mathrm{CMe}_{2}, \mathrm{CSA}$, acetone

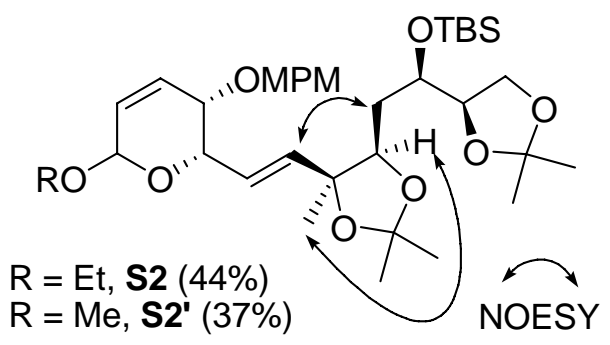


(5S, 6S, $\left.1^{\prime} E, 3^{\prime} R, 4^{\prime} R, 6^{\prime} R, 7^{\prime} R\right)$-2 -Ethoxy-5,6-dihydro-5- (4' '-methoxybenzyloxy) -6- (3' , 4', 6'-trihydroxy-7', 8' -i sopropylidenedioxy-3'-methyl-1'-octenyl) -2H-pyran<smiles>CCOC1C=C[C@H](OCC)[C@H](/C=C/[C@@](C)(O)[C@@H](O)C[C@H](O)[C@H]2COC(C)(C)O2)O1</smiles>

To a stirred solution of $15 \mathrm{a}(100 \mathrm{mg}, 0.14 \mathrm{mmol})$ in THF $(1.5 \mathrm{~mL})$ was added TBAF (1.0 $\mathrm{M}$ in THF, $340 \mathrm{~mL}, 0.34 \mathrm{mmol}$ ) at room temperature. The resultant mixture was stirred at the same temperature for $2 \mathrm{~h}$. After the addition of saturated $\mathrm{NH}_{4} \mathrm{Cl}$ solution, the reaction mixture was extracted with EtOAc. The combined organic layers were washed with $\mathrm{H}_{2} \mathrm{O}$ and saturated $\mathrm{NaCl}$ solution, dried over $\mathrm{Na}_{2} \mathrm{SO}_{4}$, and concentrated under reduced pressure. The resultant residue was purified by silica gel column chromatography (hexane $/ \mathrm{EtOAC}=1 / 1$ to EtOAc only) to afford the triol $(69 \mathrm{mg}, 100 \%$ ) as a white amorphous solid. $[\alpha]_{D}^{24}+99.5\left(C 0.51, \mathrm{CHCl}_{3}\right)$. IR $v_{\max }(\mathrm{KBr}): 3408,2980,2886,1611,1513,1370$, $1251 \mathrm{~cm}^{-1} \cdot{ }^{1} \mathrm{H}-\mathrm{NMR}\left(600 \mathrm{MHz}, \mathrm{CDCl}_{3}\right) \delta: 7.25(2 \mathrm{H}, \mathrm{d}, J=8.5 \mathrm{~Hz}), 6.87(2 \mathrm{H}, \mathrm{d}, J=8.5$ $\mathrm{Hz}), 6.11(1 \mathrm{H}, \mathrm{d}, J=10.1,5.1 \mathrm{~Hz}), 6.01(1 \mathrm{H}, \mathrm{dd}, J=10.1,2.9 \mathrm{~Hz}), 5.96(1 \mathrm{H}, \mathrm{dd}$, $J=16.0,4.3 \mathrm{~Hz}), 5.92(1 \mathrm{H}, \mathrm{d}, J=16.0 \mathrm{~Hz}), 5.12(1 \mathrm{H}, \mathrm{d}, J=2.9 \mathrm{~Hz}), 4.57(1 \mathrm{H}$, br dd, $J=4.3,2.8 \mathrm{~Hz}), 4.54(1 \mathrm{H}, \mathrm{d}, J=11.6 \mathrm{~Hz}), 4.49(1 \mathrm{H}, \mathrm{d}, J=11.6 \mathrm{~Hz}), 3.98$ $-4.02(2 \mathrm{H}, \mathrm{m}), 3.78-3.83(3 \mathrm{H}, \mathrm{m}), 3.80(3 \mathrm{H}, \mathrm{s}), 3.74(1 \mathrm{H}, \mathrm{dd}, J=10.7,9.7 \mathrm{~Hz})$, $3.66(1 \mathrm{H}, \mathrm{dd}, J=5.1,2.8 \mathrm{~Hz}), 3.56(1 \mathrm{H}, \mathrm{dq}, J=9.7,7.1 \mathrm{~Hz}), 2.72(1 \mathrm{H}, \mathrm{br} \mathrm{s}, \mathrm{OH})$, $2.51(1 \mathrm{H}$, br $\mathrm{s}, \mathrm{OH}), 2.39(1 \mathrm{H}$, br $\mathrm{s}, \mathrm{OH}), 1.64(1 \mathrm{H}, \mathrm{ddd}, J=14.1,9.6,2.0 \mathrm{~Hz})$, $1.47(1 \mathrm{H}, \mathrm{ddd}, J=14.1,10.6,2.5 \mathrm{~Hz}), 1.43(3 \mathrm{H}, \mathrm{s}), 1.36(3 \mathrm{H}, \mathrm{s}), 1.23(3 \mathrm{H}, \mathrm{s})$, $1.22(3 \mathrm{H}, t, J=7.1 \mathrm{~Hz}) \cdot{ }^{13} \mathrm{C}-\mathrm{NMR}\left(100 \mathrm{MHz}, \mathrm{CDCl}_{3}\right) \delta_{\mathrm{C}}: 159.3,136.0,130.4,129.6$, $129.4(2 \mathrm{C}), 127.2,127.2,113.8(2 \mathrm{C}), 109.5,94.2,79.2,74.8,73.3,70.4,70.4$, $69.4,68.9,66.1,63.9,55.3,34.4,26.6,25.3,22.3,15.3$. HR ESIMS m/z 531.2577 $\left(\mathrm{M}+\mathrm{Na}^{+}\right)(\mathrm{calcd}$ for 531.2564$)$.

$\left(5 S, 6 S, 1^{\prime} E, 3^{\prime} R, 4^{\prime} R, 6^{\prime} R, 7^{\prime} R\right)-6-\left(6^{\prime}-\right.$ tert-Butyldimethylsiloxy-3', 4' -dihydroxy-7', 8 ' -isopropylidenedioxy-3' -methyl-1'-octenyl)-2-ethoxy-5,6-dihydro-5- (4' '-methoxy benzyloxy) - $2 H$-pyran<smiles>CCOC1C=C[C@H](OCC)[C@H](/C=C/[C@@](C)(O)[C@H](O)CC([OH2+])[C@H]2COC(C)(C)O2)O1</smiles>

To a stirred solution of the triol $(76.1 \mathrm{mg}, 0.15 \mathrm{mmol})$ in $\mathrm{CH}_{2} \mathrm{Cl}_{2}$ (1.5 mL) was added 2,6-lutidine $(70 \mu \mathrm{l}, 0.60 \mathrm{mmol})$, followed by TBSOTf $(68 \mu \mathrm{L}, 0.30 \mathrm{mmol})$ at $-78^{\circ} \mathrm{C}$. The resultant mixture was stirred at the same temperature for 5 min. After the addition of $\mathrm{NaHCO}_{3}$ solution, the reaction mixture was extracted with EtOAc. The combined organic 
layers were washed with $\mathrm{H}_{2} \mathrm{O}$ and saturated $\mathrm{NaCl}$ solution, dried over $\mathrm{Na}_{2} \mathrm{SO}_{4}$, and concentrated under reduced pressure. The resultant residue was purified by silica gel column chromatography (hexane/EtOAc $=4 / 1$ to $2 / 1$ ) to afford the diol (57 mg,

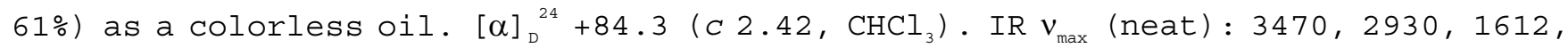
1513, 1381, $1253 \mathrm{~cm}^{-1} .{ }^{1} \mathrm{H}-\mathrm{NMR}\left(600 \mathrm{MHz}, \mathrm{CDCl}_{3}\right) \delta: 7.25(2 \mathrm{H}, \mathrm{d}, J=8.4 \mathrm{~Hz}), 6.86$ (2H, $\mathrm{d}, J=8.4 \mathrm{~Hz}), 6.09(1 \mathrm{H}, \mathrm{dd}, J=10.1,5.1 \mathrm{~Hz}), 6.01(1 \mathrm{H}, \mathrm{dd}, J=15.7,5.2 \mathrm{~Hz})$, $6.00(1 \mathrm{H}, \mathrm{dd}, J=10.1,2.9 \mathrm{~Hz}), 5.90(1 \mathrm{H}, \mathrm{d}, J=15.7 \mathrm{~Hz}), 5.11(1 \mathrm{H}, \mathrm{d}, J=2.9$ $\mathrm{Hz}), 4.56(1 \mathrm{H}, \mathrm{br} \mathrm{dd}, J=5.2,2.8 \mathrm{~Hz}), 4.53(1 \mathrm{H}, \mathrm{d}, J=11.6 \mathrm{~Hz}), 4.49(1 \mathrm{H}, \mathrm{d}, J$ $=11.6 \mathrm{~Hz}), 4.12(1 \mathrm{H}, \mathrm{q}, J=6.9 \mathrm{~Hz}), 3.97(1 \mathrm{H}, \mathrm{dt}, J=6.9,5.0 \mathrm{~Hz}), 3.95(1 \mathrm{H}, \mathrm{dd}$, $J=8.2,6.9 \mathrm{~Hz}), 3.79(1 \mathrm{H}, \mathrm{dq}, J=9.5,7.1 \mathrm{~Hz}), 3.79(3 \mathrm{H}, \mathrm{s}), 3.71(1 \mathrm{H}, t, J=$ $6.4 \mathrm{~Hz}), 3.65(1 \mathrm{H}, \mathrm{dd}, J=5.1,2.8 \mathrm{~Hz}), 3.61(1 \mathrm{H}, \mathrm{dd}, J=8.2,6.9 \mathrm{~Hz}), 3.56(1 \mathrm{H}$, $\mathrm{dq}, J=9.5,7.1 \mathrm{~Hz}), 3.20(1 \mathrm{H}$, br $\mathrm{s}, \mathrm{OH}), 2.38(1 \mathrm{H}, \mathrm{br} \mathrm{s}, \mathrm{OH}), 1.53(2 \mathrm{H}, \mathrm{dd}, J=$ $6.4,5.0 \mathrm{~Hz}), 1.41(3 \mathrm{H}, \mathrm{s}), 1.34(3 \mathrm{H}, \mathrm{s}), 1.27(3 \mathrm{H}, \mathrm{s}), 1.22(3 \mathrm{H}, \mathrm{t}, J=7.1 \mathrm{~Hz})$, $0.88(9 \mathrm{H}, \mathrm{s}), 0.11(3 \mathrm{H}, \mathrm{s}), 0.10(3 \mathrm{H}, \mathrm{s}) \cdot{ }^{13} \mathrm{C}-\mathrm{NMR}\left(100 \mathrm{MHz}, \mathrm{CDCl}_{3}\right) \delta_{\mathrm{c}}: 159.2,136.0$, $130.6,129.5,129.3(2 \mathrm{C}), 127.3,126.9,113.7(2 \mathrm{C}), 109.3,94.1,78.5,74.7,73.8$, $71.7,70.5,70.5,69.1,65.6,63.8,55.2,33.4,26.4,25.8$ (3C), 25.2, 22.2, 18.1, $15.3,-4.4,-5.0$. HR ESIMS $\mathrm{m} / \mathrm{z} 645.3438\left(\mathrm{M}+\mathrm{Na}^{+}\right)$(calcd for 645.3429).

$\left(5 S, 6 S, 1^{\prime} E, 3^{\prime} R, 4^{\prime} R, 6^{\prime} R, 7^{\prime} R\right.$ ) -6- [6'-tert-Butyldimethylsiloxy-3', 4' - 7', $8^{\prime}$-bis (isop ropylidenedioxy) -3'-methyl-1'-octenyl]-2-ethoxy-5,6-dihydro-5- (4' '-methoxybenzy loxy) - $2 H$-pyran

and

(5S, 6S, 1'E, 3'R, 4' $R, 6^{\prime} R, 7^{\prime} R$ ) -6- [6'-tert-Butyldimethylsiloxy-3' , 4' -7' , 8' -bis (isop ropylidenedioxy) -3' -methyl-1'-octenyl] -5,6-dihydro-2-methoxy-5- (4' ' -methoxybenz yloxy) -2H-pyran (S1')

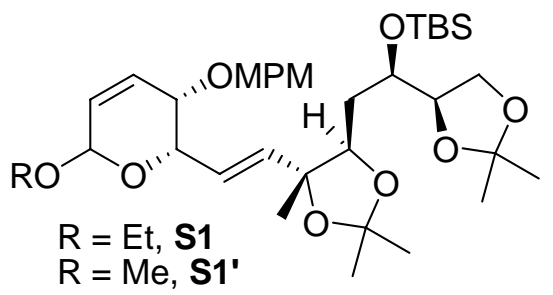

To a stirred solution of the diol (12 mg, $0.019 \mathrm{mmol})$ in acetone $(200 \mu \mathrm{L})$ was added $(\mathrm{MeO}){ }_{2} \mathrm{CMe}_{2}(100 \mu \mathrm{L})$, followed by CSA $(0.5 \mathrm{mg}, 0.02 \mathrm{mmol})$ at room temperature. The resultant mixture was stirred at the same temperature for $2.5 \mathrm{~h}$. After the addition of $\mathrm{NaHCO}_{3}$ solution, the reaction mixture was extracted with EtOAC. The combined organic layers were washed with $\mathrm{H}_{2} \mathrm{O}$ and saturated $\mathrm{NaCl}$ solution, dried over $\mathrm{Na}_{2} \mathrm{SO}_{4}$, and concentrated under reduced pressure. The resultant residue was purified by silica gel PTLC (hexane/EtOAC $=3 / 1$ ) to afford s1 (4.2 mg, 33\%) and s1' (4.6 mg, 37\%) as a colorless oil, respectively. S1: $[\alpha]_{D}{ }^{24}+67.1\left(\mathrm{C} 0.34, \mathrm{CHCl}_{3}\right.$ ). IR $v_{\max }$ (neat): 2985, 2931, 1612, 1513, 1380, 1252, 1110, 837, $778 \mathrm{~cm}^{-1} \cdot{ }^{1} \mathrm{H}-\mathrm{NMR}\left(600 \mathrm{MHz}, \mathrm{CDCl}_{3}\right) \delta: 7.25$ 
$(2 \mathrm{H}, \mathrm{d}, J=8.3 \mathrm{~Hz}), 6.85(2 \mathrm{H}, \mathrm{d}, J=8.3 \mathrm{~Hz}), 6.07(1 \mathrm{H}, \mathrm{dd}, J=10.2,5.5 \mathrm{~Hz}), 6.05$ $(1 \mathrm{H}, \mathrm{dd}, J=15.9,5.9 \mathrm{~Hz}), 5.98(1 \mathrm{H}, \mathrm{dd}, J=10.2,2.9 \mathrm{~Hz}), 5.86(1 \mathrm{H}, \mathrm{d}, J=15.9$ $\mathrm{Hz}), 5.10(1 \mathrm{H}, \mathrm{d}, J=2.9 \mathrm{~Hz}), 4.50-4.56(1 \mathrm{H}, \mathrm{m}), 4.52(1 \mathrm{H}, \mathrm{d}, J=11.4 \mathrm{~Hz}), 4.49$ $(1 \mathrm{H}, \mathrm{d}, J=11.4 \mathrm{~Hz}), 4.01-4.07(2 \mathrm{H}, \mathrm{m}), 3.93-3.98(2 \mathrm{H}, \mathrm{m}), 3.75-3.82(2 \mathrm{H}$, $\mathrm{m}), 3.80(3 \mathrm{H}, \mathrm{s}), 3.63(1 \mathrm{H}, \mathrm{dd}, J=5.5,2.7 \mathrm{~Hz}), 3.54(1 \mathrm{H}, \mathrm{dq}, J=9.3,7.2 \mathrm{~Hz})$, $1.50-1.55(1 \mathrm{H}, \mathrm{m}), 1.47(3 \mathrm{H}, \mathrm{s}), 1.43(3 \mathrm{H}, \mathrm{s}), 1.37-1.42(1 \mathrm{H}, \mathrm{m}), 1.37(3 \mathrm{H}, \mathrm{s})$, $1.34(3 \mathrm{H}, \mathrm{s}), 1.21(3 \mathrm{H}, \mathrm{s}), 1.21(3 \mathrm{H}, \mathrm{t}, J=7.2 \mathrm{~Hz}), 0.86(9 \mathrm{H}, \mathrm{s}), 0.09(3 \mathrm{H}, \mathrm{s})$, $0.08(3 \mathrm{H}, \mathrm{s}) \cdot{ }^{13} \mathrm{C}-\mathrm{NMR}\left(100 \mathrm{MHz}, \mathrm{CDCl}_{3}\right) \delta_{\mathrm{C}}: 159.1,134.8,130.7,129.3,129.2$ (2C), $127.5,127.3,113.7(2 \mathrm{C}), 109.3,107.6,94.1,81.8,78.7,78.1,70.8,70.8,70.4$, $69.3,65.3,63.7,55.2,31.7,28.6,27.0,26.4,25.9$ (3C), 25.1, 20.8, 18.1, 15.4, $-4.0,-4.8$. HR ESIMS m/z $685.3725\left(\mathrm{M}+\mathrm{Na}^{+}\right)$(calcd for 685.3742).

S1': $[\alpha]_{D}^{24}+67.5\left(\mathrm{C} 0.39, \mathrm{CHCl}_{3}\right)$. IR $v_{\max }$ (neat): 2985, 2931, 1612, 1513, 1380, 1252, 1110, 837, $778 \mathrm{~cm}^{-1} .{ }^{1} \mathrm{H}-\mathrm{NMR}\left(600 \mathrm{MHz}, \mathrm{CDCl}_{3}\right) \delta: 7.25(2 \mathrm{H}, \mathrm{d}, J=8.4 \mathrm{~Hz}), 6.85(2 \mathrm{H}$, $\mathrm{d}, J=8.4 \mathrm{~Hz}), 6.07(1 \mathrm{H}, \mathrm{dd}, J=10.1,5.0 \mathrm{~Hz}), 6.05(1 \mathrm{H}, \mathrm{dd}, J=15.7,6.0 \mathrm{~Hz})$, $5.98(1 \mathrm{H}, \mathrm{dd}, J=10.1,2.9 \mathrm{~Hz}), 5.87(1 \mathrm{H}, \mathrm{d}, J=15.7 \mathrm{~Hz}), 4.97(1 \mathrm{H}, \mathrm{d}, J=2.9$ $\mathrm{Hz}), 4.52(1 \mathrm{H}, \mathrm{d}, J=11.6 \mathrm{~Hz}), 4.46-4.52(2 \mathrm{H}, \mathrm{m}), 4.02-4.08(2 \mathrm{H}, \mathrm{m}), 3.92-$ $3.98(2 \mathrm{H}, \mathrm{m}), 3.80(3 \mathrm{H}, \mathrm{s}), 3.76(1 \mathrm{H}, \mathrm{t}, J=7.6 \mathrm{~Hz}), 3.62(1 \mathrm{H}, \mathrm{dd}, J=5.0,2.8$ $\mathrm{Hz}), 3.40(3 \mathrm{H}, \mathrm{s}), 1.61(3 \mathrm{H}, \mathrm{s}), 1.51-1.56(1 \mathrm{H}, \mathrm{m}), 1.37-1.43(1 \mathrm{H}, \mathrm{m}), 1.43(3 \mathrm{H}$, $\mathrm{s}), 1.37(3 \mathrm{H}, \mathrm{s}), 1.35(3 \mathrm{H}, \mathrm{s}), 1.22(3 \mathrm{H}, \mathrm{s}), 0.86(9 \mathrm{H}, \mathrm{s}), 0.09(3 \mathrm{H}, \mathrm{s}), 0.08(3 \mathrm{H}$, s) $.{ }^{13} \mathrm{C}-\mathrm{NMR}\left(100 \mathrm{MHz}, \mathrm{CDCl}_{3}\right) \delta_{\mathrm{C}}: 159.1,135.1,130.7,129.3(2 \mathrm{C}), 129.0,127.6,127.1$, $113.7(2 \mathrm{C}), 109.3,107.6,95.2,81.8,78.7,78.1,70.8(2 \mathrm{C}), 70.4,69.2,65.3,55.5$, $55.2,31.8,28.6,27.0,26.4,25.9$ (3C), 25.1, 20.7, 18.1, -4.0,-4.8. HR ESIMS m/z $671.3588\left(\mathrm{M}+\mathrm{Na}^{+}\right)(\mathrm{calcd}$ for 671.3585$)$.

$\left(5 S, 6 S, 1^{\prime} E, 3^{\prime} R, 4^{\prime} R, 6^{\prime} R, 7^{\prime} R\right)-2$-Ethoxy-5,6-dihydro-5- (4' '-methoxybenzyloxy) -6- (3' , 4', 6' -trihydroxy-7', 8' -i sopropylidenedioxy-3'-methyl-1'-octenyl) -2H-pyran<smiles>CCOC1C=C[C@H](OCC)[C@H](/C=C/[C@@](C)(O)[C@@H](O)C[C@H](O)[C@H]2COC(C)(C)O2)O1</smiles>

15b (100 mg, $0.136 \mathrm{mmol}$ ) was treated according to the same procedure as that described above to give crude triol 15a, which was purified by silica gel column chromatography (hexane/EtOAC = 1/1 to EtOAC only) to afford the triol (29.9 mg, 42\%) as a white amorphous solid. $[\alpha]_{\mathrm{D}}^{24}+107.2\left(\mathrm{C} 0.70, \mathrm{CHCl}_{3}\right)$. IR $v_{\max }(\mathrm{KBr}): 3452,2925,2875,1612$, 1514, 1442, 1369, 1257, 1110, $835 \mathrm{~cm}^{-1} \cdot{ }^{1} \mathrm{H}-\mathrm{NMR}\left(600 \mathrm{MHz}, \mathrm{CDCl}_{3}\right) \delta: 7.24(2 \mathrm{H}, \mathrm{d}, \mathrm{J}=$ $8.4 \mathrm{~Hz}), 6.86(2 \mathrm{H}, \mathrm{d}, J=8.4 \mathrm{~Hz}), 6.12(1 \mathrm{H}, \mathrm{dd}, J=10.1,5.1 \mathrm{~Hz}), 6.01(1 \mathrm{H}, \mathrm{dd}$, $J=10.1,2.9 \mathrm{~Hz}), 5.95(1 \mathrm{H}, \mathrm{dd}, J=15.8,4.3 \mathrm{~Hz}), 5.90(1 \mathrm{H}, \mathrm{d}, J=15.8 \mathrm{~Hz}), 5.12$ 
$(1 \mathrm{H}, \mathrm{d}, J=2.9 \mathrm{~Hz}), 4.59(1 \mathrm{H}, \mathrm{br} d d, J=4.3,2.8 \mathrm{~Hz}), 4.54(1 \mathrm{H}, \mathrm{d}, J=11.6 \mathrm{~Hz})$, $4.48(1 \mathrm{H}, \mathrm{d}, J=11.6 \mathrm{~Hz}), 3.91-3.97(2 \mathrm{H}, \mathrm{m}), 3.77-3.83(2 \mathrm{H}, \mathrm{m}), 3.78(3 \mathrm{H}, \mathrm{m})$, $3.69-3.74(1 \mathrm{H}, \mathrm{m}), 3.69(1 \mathrm{H}, \mathrm{t}, J=5.8 \mathrm{~Hz}), 3.65(1 \mathrm{H}, \mathrm{dd}, J=5.1,2.8 \mathrm{~Hz}), 3.57$ $(1 \mathrm{H}, \mathrm{dq}, J=9.6,7.1 \mathrm{~Hz}), 2.65(1 \mathrm{H}, \mathrm{br} \mathrm{s}, \mathrm{OH}), 2.57(1 \mathrm{H}, \mathrm{br} \mathrm{s}, \mathrm{OH}), 2.45(1 \mathrm{H}, \mathrm{br}$ $\mathrm{s}, \mathrm{OH}), 1.65(1 \mathrm{H}, \mathrm{ddd}, J=13.9,9.6,1.9 \mathrm{~Hz}), 1.42(3 \mathrm{H}, \mathrm{s}), 1.39(1 \mathrm{H}, \mathrm{ddd}, J=13.9$, $10.4,2.3 \mathrm{~Hz}), 1.34(3 \mathrm{H}, \mathrm{s}), 1.34(3 \mathrm{H}, \mathrm{s}), 1.22(3 \mathrm{H}, \mathrm{t}, J=7.1 \mathrm{~Hz}) .{ }^{13} \mathrm{C}-\mathrm{NMR}(100$ $\left.\mathrm{MHz}, \mathrm{CDCl}_{3}\right) \delta_{\mathrm{C}}: 159.3,133.8,130.3,129.7,129.4(2 \mathrm{C}), 127.2,127.1,113.8(2 \mathrm{C}), 109.5$, $94.2,79.0,74.8,74.3,70.3,70.2,69.2,68.7,66.0,63.9,55.2,35.6,26.6,25.3$, 24.5, 15.3. HR ESIMS m/z $531.2551\left(\mathrm{M}+\mathrm{Na}^{+}\right)$(calcd for 531.2564).

$\left(5 S, 6 S, 1^{\prime} E, 3^{\prime} R, 4^{\prime} S, 6^{\prime} R, 7^{\prime} R\right)-6-\left(6^{\prime}-\right.$ tert-Butyldimethylsiloxy-3' , 4' -dihydroxy-7', 8 '-isopropylidenedioxy-3' -methyl-1'-octenyl) -5,6-dihydro-2-ethoxy-5- (4' ' -methoxy benzyloxy) - $2 H$-pyran<smiles>CCOC1C=C[C@H](OCC)[C@H](/C=C/[C@@](C)(O)[C@@H](O)CC([OH2+])C2COC(C)(C)O2)O1</smiles>

The triol (18.9 $\mathrm{mg}, 0.037 \mathrm{mmol}$ ) was treated according to the same procedure as that described above, which was purified by silica gel column chromatography (hexane/EtOAC $=4 / 1$ to $2 / 1$ ) to afford the diol $\left(16.7 \mathrm{mg}, 72 \%\right.$ ) as a white amorphous solid. $[\alpha]_{\mathrm{D}}^{24}$ $+82.1\left(\mathrm{C} 0.4, \mathrm{CHCl}_{3}\right)$. IR $v_{\max }(\mathrm{film}): 2930,1612,1513,1381,1252 \mathrm{~cm}^{-1} \cdot{ }^{1} \mathrm{H}-\mathrm{NMR}$ (600 $\left.\mathrm{MHz}, \mathrm{CDCl}_{3}\right) \delta: 7.25(2 \mathrm{H}, \mathrm{d}, J=8.5 \mathrm{~Hz}), 6.86(2 \mathrm{H}, \mathrm{d}, J=8.5 \mathrm{~Hz}), 6.11(1 \mathrm{H}, \mathrm{dd}, J$ $=10.1,5.1 \mathrm{~Hz}), 6.00(1 \mathrm{H}, \mathrm{dd}, J=10.1,2.9 \mathrm{~Hz}), 5.97(1 \mathrm{H}, \mathrm{dd}, J=15.8,5.2 \mathrm{~Hz})$, $5.86(1 \mathrm{H}, \mathrm{dd}, J=15.8,1.2 \mathrm{~Hz}), 5.10(1 \mathrm{H}, \mathrm{d}, J=2.9 \mathrm{~Hz}), 4.56(1 \mathrm{H}, \mathrm{br} \mathrm{ddd}, J=$ $5.2,2.8,1.2 \mathrm{~Hz}), 4.54(1 \mathrm{H}, \mathrm{d}, J=11.6 \mathrm{~Hz}), 4.47(1 \mathrm{H}, \mathrm{d}, J=11.6 \mathrm{~Hz}), 4.09(1 \mathrm{H}$, $q, J=6.8 \mathrm{~Hz}), 3.86-3.91(2 \mathrm{H}, \mathrm{m}), 3.80(1 \mathrm{H}, \mathrm{dq}, J=9.8,7.1 \mathrm{~Hz}), 3.80(3 \mathrm{H}, \mathrm{s})$, $3.63-3.67(1 \mathrm{H}, \mathrm{m}), 3.64(1 \mathrm{H}, \mathrm{dd}, J=5.1,2.8 \mathrm{~Hz}), 3.52-3.57(2 \mathrm{H}, \mathrm{m}), 3.10(1 \mathrm{H}$, br $d, J=4.7 \mathrm{~Hz}, \mathrm{OH}), 2.54(1 \mathrm{H}, \mathrm{br} \mathrm{s}, \mathrm{OH}), 1.48-1.54(2 \mathrm{H}, \mathrm{m}), 1.34(3 \mathrm{H}, \mathrm{s}), 1.32$ $(3 \mathrm{H}, \mathrm{s}), 1.32(3 \mathrm{H}, \mathrm{s}), 1.22(3 \mathrm{H}, \mathrm{t}, J=7.1 \mathrm{~Hz}), 0.89(9 \mathrm{H}, \mathrm{s}), 0.10(3 \mathrm{H}, \mathrm{s}), 0.10$ $(3 \mathrm{H}, \mathrm{s}) \cdot{ }^{13} \mathrm{C}-\mathrm{NMR}\left(100 \mathrm{MHz}, \mathrm{CDCl}_{3}\right) \delta_{\mathrm{C}}: 159.2,133.9,130.5,129.6,129.3(2 \mathrm{C}), 127.2$, $127.0,113.8(2 \mathrm{C}), 109.3,94.1,78.4,74.7,74.5,71.5,70.4,70.4,68.9,65.5,63.8$, $55.2,34.2,26.4,25.9$ (3C) $, 25.2,24.5,18.1,15.4,-4.4,-4.9$. HR ESIMS m/z 645.3419 $\left(\mathrm{M}+\mathrm{Na}^{+}\right)(\mathrm{calcd}$ for 645.3429$)$.

$\left(5 S, 6 S, 1^{\prime} E, 3^{\prime} S, 4^{\prime} R, 6^{\prime} R, 7^{\prime} R\right)-6$ - [6'-tert-Butyldimethylsiloxy-3', 4' - 7' , 8' -bis (isop ropylidenedioxy) -3' -methyl-1'-octenyl]-2-ethoxy-5,6-dihydro-5- (4' '-methoxybenzy 
$\left(5 S, 6 S, 1^{\prime} E, 3^{\prime} S, 4^{\prime} R, 6^{\prime} R, 7^{\prime} R\right)-6$ - [6'-tert-Butyldimethylsiloxy-3', 4' - 7' , 8'-bis (isop ropylidenedioxy) -3' -methyl-1'-octenyl] -5,6-dihydro-2-methoxy-5- (4' '-methoxybenz yloxy) -2H-pyran ( $\left.\mathrm{S2}^{\prime}\right)$

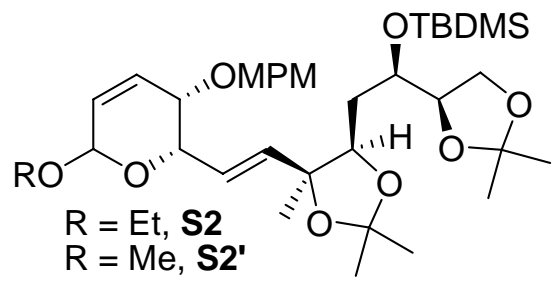

The diol (10.0 mg, $0.016 \mathrm{mmol}$ ) was treated according to the same procedure as that described above to give crude acetal s1 and S1', which was purified by silica gel PTLC (hexane/EtOAC $=3 / 1)$ to afford $\mathrm{s} 2(3.9 \mathrm{mg}, 37 \%)$ and $\mathrm{s} 2{ }^{\prime} \mathrm{b}(4.8 \mathrm{mg}, 44 \%)$ as a colorless oil. S2: $[\alpha]_{D}^{24}+72.6\left(\mathrm{C} 0.27, \mathrm{CHCl}_{3}\right)$. IR $v_{\max }$ (neat): 2982, 1612, 1513, 1380, $1251 \mathrm{~cm}^{-1} \cdot{ }^{1} \mathrm{H}-\mathrm{NMR}\left(600 \mathrm{MHz}, \mathrm{CDCl}_{3}\right) \delta: 7.26(2 \mathrm{H}, \mathrm{d}, J=8.3 \mathrm{~Hz}), 6.85(2 \mathrm{H}, \mathrm{d}, \mathrm{J}=8.3$ $\mathrm{Hz}), 6.07(1 \mathrm{H}, \mathrm{dd}, J=10.1,5.2 \mathrm{~Hz}), 6.00(1 \mathrm{H}, \mathrm{dd}, J=10.1,2.9 \mathrm{~Hz}), 5.99(1 \mathrm{H}$, $\mathrm{dd}, J=15.7,5.7 \mathrm{~Hz}), 5.79(1 \mathrm{H}, \mathrm{d}, J=15.7 \mathrm{~Hz}), 5.09(1 \mathrm{H}, \mathrm{d}, J=2.9 \mathrm{~Hz}), 4.53$ $(1 \mathrm{H}, \mathrm{dd}, J=5.7,2.8 \mathrm{~Hz}), 4.50(1 \mathrm{H}, \mathrm{d}, J=11.4 \mathrm{~Hz}), 4.47(1 \mathrm{H}, \mathrm{d}, J=11.4 \mathrm{~Hz})$, $4.08(1 \mathrm{H}, \mathrm{d}, J=10.5 \mathrm{~Hz}), 3.94(1 \mathrm{H}, \mathrm{q}, J=6.7 \mathrm{~Hz}), 3.89(1 \mathrm{H}, \mathrm{dd}, J=10.1,6.7$ $\mathrm{Hz}), 3.83(1 \mathrm{H}, \mathrm{dd}, J=8.1,7.1 \mathrm{~Hz}), 3.80(3 \mathrm{H}, \mathrm{s}), 3.78(1 \mathrm{H}, \mathrm{dq}, J=9.7,6.7 \mathrm{~Hz})$, $3.63(1 \mathrm{H}, \mathrm{dd}, J=5.2,2.8 \mathrm{~Hz}), 3.60(1 \mathrm{H}, \mathrm{dd}, J=8.1,7.1 \mathrm{~Hz}), 3.55(1 \mathrm{H}, \mathrm{dq}, J=$ $9.7,6.7 \mathrm{~Hz}), 1.51(3 \mathrm{H}, \mathrm{s}), 1.42-1.48(1 \mathrm{H}, \mathrm{m}), 1.41(3 \mathrm{H}, \mathrm{s}), 1.31-1.39(1 \mathrm{H}, \mathrm{m})$, $1.38(3 \mathrm{H}, \mathrm{s}), 1.35(3 \mathrm{H}, \mathrm{s}), 1.30(3 \mathrm{H}, \mathrm{s}), 1.22(3 \mathrm{H}, \mathrm{t}, J=7.1 \mathrm{~Hz}), 0.90(9 \mathrm{H}, \mathrm{s})$, $0.11(3 \mathrm{H}, \mathrm{s}), 0.11(3 \mathrm{H}, \mathrm{s}) \cdot{ }^{13} \mathrm{C}-\mathrm{NMR}\left(100 \mathrm{MHz}, \mathrm{CDCl}_{3}\right) \delta_{\mathrm{C}}: 159.1,133.5,130.7,129.6$ (2C) $129.4,127.5,127.0,113.7(2 \mathrm{C}), 109.2,107.9,94.1,81.8,79.9,78.9,70.8$, $70.5,70.3,69.3,65.3,63.7,55.2,28.4,27.0,26.4,26.0$ (3C), 25.9, 25.2, 24.0, 18.2, 15.4, -3.9, -4.8. HR ESIMS m/z $685.3743\left(\mathrm{M}^{\left.+\mathrm{Na}^{+}\right)}(\mathrm{calcd}\right.$ for 685.3742).

S2': $[\alpha]_{D}^{24}+73.6\left(C 0.38, \mathrm{CHCl}_{3}\right)$. IR $v_{\max }$ (neat) : 2982, 1612, 1513, 1380, 1251, 1094 $\mathrm{Cm}^{-1} .{ }^{1} \mathrm{H}-\mathrm{NMR}\left(600 \mathrm{MHz}, \mathrm{CDCl}_{3}\right) \delta: 7.26(2 \mathrm{H}, \mathrm{d}, J=8.6 \mathrm{~Hz}), 6.85(2 \mathrm{H}, \mathrm{d}, J=8.6 \mathrm{~Hz})$, $6.07(1 \mathrm{H}, \mathrm{dd}, J=10.0,5.2 \mathrm{~Hz}), 6.00(1 \mathrm{H}, \mathrm{dd}, J=15.8,6.1 \mathrm{~Hz}), 5.99(1 \mathrm{H}, \mathrm{dd}, J$ $=10.0,2.9 \mathrm{~Hz}), 5.81(1 \mathrm{H}, \mathrm{dd}, J=15.8,1.1 \mathrm{~Hz}), 4.97(1 \mathrm{H}, \mathrm{d}, J=2.9 \mathrm{~Hz}), 4.50$ $(1 \mathrm{H}, \mathrm{d}, J=11.4 \mathrm{~Hz}), 4.47-4.50(1 \mathrm{H}, \mathrm{m}), 4.48(1 \mathrm{H}, \mathrm{d}, J=11.4 \mathrm{~Hz}), 4.08(1 \mathrm{H}, \mathrm{dd}$, $J=10.6,1.1 \mathrm{~Hz}), 3.96(1 \mathrm{H}, \mathrm{q}, J=6.6 \mathrm{~Hz}), 3.90(1 \mathrm{H}, \mathrm{ddd}, J=10.6,6.6,1.1 \mathrm{~Hz})$, $3.84(1 \mathrm{H}, \mathrm{dd}, J=8.2,6.6 \mathrm{~Hz}), 3.80(3 \mathrm{H}, \mathrm{s}), 3.60-3.63(1 \mathrm{H}, \mathrm{m}), 3.62(1 \mathrm{H}, \mathrm{dd}$, $J=8.2,6.6 \mathrm{~Hz}), 3.40(3 \mathrm{H}, \mathrm{s}), 1.50(3 \mathrm{H}, \mathrm{s}), 1.46(1 \mathrm{H}, \mathrm{ddd}, J=14.0,10.6,1.1$ $\mathrm{Hz}), 1.41(3 \mathrm{H}, \mathrm{s}), 1.38(3 \mathrm{H}, \mathrm{s}), 1.36(3 \mathrm{H}, \mathrm{s}), 1.32-1.36(1 \mathrm{H}, \mathrm{m}), 1.30(3 \mathrm{H}, \mathrm{s})$, $0.90(9 \mathrm{H}, \mathrm{s}), 0.11(3 \mathrm{H}, \mathrm{s}), 0.11(3 \mathrm{H}, \mathrm{s}) \cdot{ }^{13} \mathrm{C}-\mathrm{NMR}\left(100 \mathrm{MHz}, \mathrm{CDCl}_{3}\right) \delta_{\mathrm{c}}: 159.2,133.7$, $130.7,129.4$ (2C) $129.3,127.6,126.9,113.7(2 \mathrm{C}), 109.2,108.0,95.2,81.8,79.9$, $78.8,70.9,70.5,70.3,69.2,65.2,55.5,55.2,33.2,28.4,27.0,26.4,26.0(4 \mathrm{C})$ ， 
25.1, 24.1, 18.2, -3.9, -4.8. HR ESIMS m/z 671.3628 (M+Na ${ }^{+}$(calcd for 671.3591).

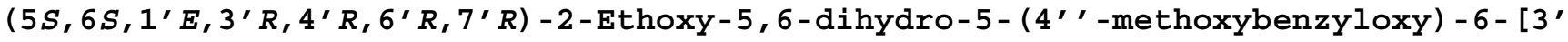
'4' , 6'-tris (tert-butyldimethylsiloxy) -7' , 8' -i sopropylidenedioxy-3'-methyl-1'-oc tenyl] -2H-pyran

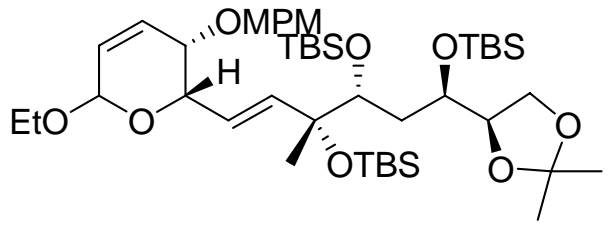

To a stirred solution of $15 \mathrm{a}(2.49 \mathrm{~g}, 3.38 \mathrm{mmol})$ in $\mathrm{CH}_{2} \mathrm{Cl}_{2}(17 \mathrm{~mL}$ ) was added $2,6-1$ utidine (1.2 mL, $10.3 \mathrm{mmol})$, followed by TBSOTf $(1.9 \mathrm{~mL}, 8.4 \mathrm{mmol})$ at $0{ }^{\circ} \mathrm{C}$. The resultant mixture was stirred at room temperature for $1 \mathrm{~h}$. After the addition of $\mathrm{NaHCO}_{3}$ solution, the reaction mixture was extracted with EtOAc. The combined organic layers were washed with $\mathrm{H}_{2} \mathrm{O}$ and saturated $\mathrm{NaCl}$ solution, dried over $\mathrm{Na}_{2} \mathrm{SO}_{4}$, and concentrated under reduced pressure. The resultant residue was purified by silica gel column chromatography (hexane/EtOAC $=9 / 1)$ to afford the TBS ether $(2.70 \mathrm{~g}, 94 \%)$ as a colorless oil. $[\alpha]_{\mathrm{D}}^{24}$ $+126.1\left(\mathrm{C} 0.73, \mathrm{CHCl}_{3}\right)$. IR $v_{\max }$ (neat): 2955, 1514, 1252, $1095 \mathrm{~cm}^{-1} \cdot{ }^{1} \mathrm{H}-\mathrm{NMR}(600 \mathrm{MHz}$, $\left.\mathrm{CDCl}_{3}\right) \delta: 7.24(2 \mathrm{H}, \mathrm{d}, J=8.6 \mathrm{~Hz}), 6.85(2 \mathrm{H}, \mathrm{d}, J=8.6 \mathrm{~Hz}), 6.06(1 \mathrm{H}, \mathrm{dd}, J=10.3$, $5.1 \mathrm{~Hz}), 5.98(1 \mathrm{H}, \mathrm{dd}, J=10.3,2.9 \mathrm{~Hz}), 5.97(1 \mathrm{H}, \mathrm{d}, J=15.9 \mathrm{~Hz}), 5.88(1 \mathrm{H}, \mathrm{dd}$, $J=15.9,6.0 \mathrm{~Hz}), 5.09(1 \mathrm{H}, \mathrm{d}, J=2.9 \mathrm{~Hz}), 4.49-4.55(3 \mathrm{H}, \mathrm{m}), 3.87(1 \mathrm{H}, \mathrm{q}, J$ $=7.0 \mathrm{~Hz}), 3.80(3 \mathrm{H}, \mathrm{s}), 3.77-3.84(3 \mathrm{H}, \mathrm{m}), 3.70(1 \mathrm{H}, \mathrm{dd}, J=8.5,1.3 \mathrm{~Hz}), 3.64(1 \mathrm{H}$, $\mathrm{dd}, J=5.1,3.0 \mathrm{~Hz}), 3.51-3.57(2 \mathrm{H}, \mathrm{m}), 1.88(1 \mathrm{H}, \mathrm{ddd}, J=13.9,10.1,1.3 \mathrm{~Hz})$, $1.39(3 \mathrm{H}, \mathrm{s}), 1.37(3 \mathrm{H}, \mathrm{s}), 1.30(3 \mathrm{H}, \mathrm{s}), 1.21(3 \mathrm{H}, \mathrm{t}, \mathrm{J}=7.1 \mathrm{~Hz}), 1.12$ ( $1 \mathrm{H}$, ddd, $J=13.9,8.5,1.3 \mathrm{~Hz}), 0.89(27 \mathrm{H}, \mathrm{s}), 0.12(3 \mathrm{H}, \mathrm{s}), 0.12(3 \mathrm{H}, \mathrm{s}), 0.11(3 \mathrm{H}, \mathrm{s}), 0.11(3 \mathrm{H}$, s), $0.07(3 \mathrm{H}, \mathrm{s}), 0.07(3 \mathrm{H}, \mathrm{s}) \cdot{ }^{13} \mathrm{C}-\mathrm{NMR}\left(100 \mathrm{MHz}, \mathrm{CDCl}_{3}\right) \delta_{\mathrm{C}}: 159.1,136.8,130.9,129.6$, $129.2(2 \mathrm{C}), 127.6,125.9,113.7(2 \mathrm{C}), 109.2,94.0,79.5,78.0,77.0,71.5,71.3$, $70.3,69.5,65.8,63.6,55.2,37.2,26.5,26.2$ (3C), 26.2 (3C), 26.1 (3C), 25.4, $25.3,18.4,18.4,18.3,15.3,-1.6,-1.7,-3.0,-3.6,-3.6,-3.9$. HR ESIMS m/z $873.5164\left(\mathrm{M}+\mathrm{Na}^{+}\right)(\mathrm{calcd}$ for 873.5158$)$.

(5S, 6S, 1' $\left.E, 3^{\prime} R, 4^{\prime} R, 6^{\prime} R, 7{ }^{\prime} R\right)$-5, 6-Dihydro-2-hydroxy-5- (4' ' -methoxybenzyloxy) - 6- [3 ' , 4', 6'-tris (tert-butyldimethylsiloxy) -7', 8' -isopropylidenedioxy-3' -methyl-1' -o ctenyl]-2H-pyran

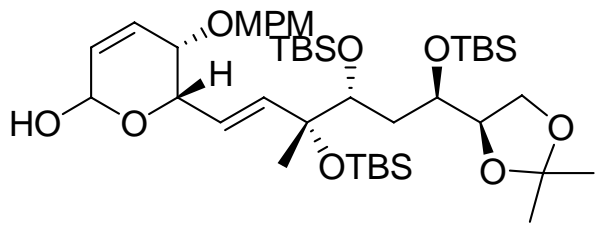


To a stirred solution of the ethylacetal (256 mg, $0.30 \mathrm{mmol}$ ) in MeCN (6 mL) was added $\mathrm{Zn}\left(\mathrm{NO}_{3}\right)_{2} \cdot 6 \mathrm{H}_{2} \mathrm{O}(892 \mathrm{mg}, 3.0 \mathrm{mmol})$ at $60^{\circ} \mathrm{C}$. The resultant mixture was stirred at the same temperature for $7 \mathrm{~min}$. After the addition of EtOAc and $\mathrm{H}_{2} \mathrm{O}$, the reaction mixture was extracted with EtOAc. The combined organic layers were washed with $\mathrm{H}_{2} \mathrm{O}$ and saturated $\mathrm{NaCl}$ solution, dried over $\mathrm{Na}_{2} \mathrm{SO}_{4}$, and concentrated under reduced pressure. The resultant residue was purified by silica gel column chromatography (hexane/EtOAc $=9 / 1$ to $4 / 1$ ) to afford hemiacetal (162 mg, 66\%) as a colorless oil and the recovered ethyl acetal $(48 \mathrm{mg}, 19 \%) .[\alpha]_{\mathrm{D}}^{24}+66.4\left(\mathrm{C} 0.093, \mathrm{CHCl}_{3}\right)$. IR $v_{\max }$ (neat): 2955, 1613, 1253, $1095 \mathrm{~cm}^{-1} .{ }^{1} \mathrm{H}-\mathrm{NMR}\left(600 \mathrm{MHz}, \mathrm{CDCl}_{3}\right) \delta: 7.25(2 \mathrm{H}, \mathrm{d}, J=8.6 \mathrm{~Hz}), 6.85(2 \mathrm{H}, \mathrm{d}$, $J=8.6 \mathrm{~Hz}), 6.08(1 \mathrm{H}, \mathrm{dd}, J=10.2,5.2 \mathrm{~Hz}), 6.01(1 \mathrm{H}, \mathrm{dd}, J=10.2,2.9 \mathrm{~Hz}), 5.97$ $(1 \mathrm{H}, \mathrm{d}, J=15.9 \mathrm{~Hz}), 5.93(1 \mathrm{H}, \mathrm{dd}, J=15.9,5.5 \mathrm{~Hz}), 5.51(1 \mathrm{H}, \mathrm{br} \mathrm{dd}, J=4.2$ ， $2.9 \mathrm{~Hz}), 5.60(1 \mathrm{H}, \mathrm{dd}, J=5.5,2.5 \mathrm{~Hz}), 4.54(1 \mathrm{H}, \mathrm{d}, J=11.6 \mathrm{~Hz}), 4.50(1 \mathrm{H}, \mathrm{d}$, $J=11.6 \mathrm{~Hz}), 3.80(3 \mathrm{H}, \mathrm{s}), 3.75-3.90(3 \mathrm{H}, \mathrm{m}), 3.68(1 \mathrm{H}, \mathrm{dd}, J=8.7,1.1 \mathrm{~Hz})$, $3.61(1 \mathrm{H}, \mathrm{d}, J=5.2,2.5 \mathrm{~Hz}), 3.55(1 \mathrm{H}, t, J=7.8 \mathrm{~Hz}), 2.60(1 \mathrm{H}, \mathrm{d}, J=4.2 \mathrm{~Hz})$, $1.85(1 \mathrm{H}, \mathrm{ddd}, J=14.1,10.3,1.1 \mathrm{~Hz}), 1.37(3 \mathrm{H}, \mathrm{s}), 1.37(3 \mathrm{H}, \mathrm{s}), 1.30(3 \mathrm{H}, \mathrm{s})$, $1.16(1 \mathrm{H}, \mathrm{ddd}, J=14.1,8.7,1.3 \mathrm{~Hz}), 0.89(18 \mathrm{H}, \mathrm{s}), 0.88(9 \mathrm{H}, \mathrm{s}), 0.12(3 \mathrm{H}, \mathrm{s})$, $0.11(6 \mathrm{H}, \mathrm{s}), 0.10(3 \mathrm{H}, \mathrm{s}), 0.08(3 \mathrm{H}, \mathrm{s}), 0.08(3 \mathrm{H}, \mathrm{s}) \cdot{ }^{13} \mathrm{C}-\mathrm{NMR}\left(100 \mathrm{MHz}, \mathrm{CDCl}_{3}\right) \delta_{\mathrm{C}}$ : $159.1,138.6,129.7,129.6,129.2$ (2C), 127.9, 125.2, 113.7 (2C), 109.2, 88.8, 79.5, $77.7,76.9,72.0,71.5,70.7,69.6,65.8,55.2,37.2,26.5,26.2$ (3C), 26.2 (3C), $26.2(3 \mathrm{C}), 25.4,25.3,18.5,18.4,18.4,-1.5,-1.6,-2.9,-3.5,-3.6,-3.8$. HR ESIMS $\mathrm{m} / \mathrm{z} 845.4876\left(\mathrm{M}+\mathrm{Na}^{+}\right)(\mathrm{calcd}$ for 845.4845$)$.

(5S, 6S, 1'E, 3' $\left.R, 4^{\prime} R, 6^{\prime} R, 7^{\prime} R\right)-5,6$-Dihydro-5-(4' '-methoxybenzyloxy) -6- [3', 4', 6'-tr is (tert-butyldimethylsiloxy) - 7', 8' -isopropylidenedioxy-3'-methyl-1'-octenyl] -2H -pyran-2-one (16)

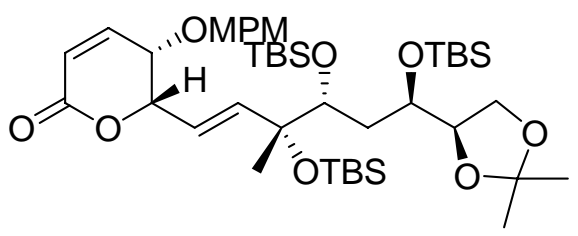

To a stirred solution of the hemiacetal $\left(640 \mathrm{mg}, 0.78 \mathrm{mmol}\right.$ ) in $\mathrm{CH}_{2} \mathrm{Cl}_{2}$ (16 $\mathrm{mL}$ ) was added $\mathrm{MnO}_{2}(1.35 \mathrm{~g}, 15.5 \mathrm{mmol})$ at room temperature. After stirred overnight, the reaction mixture was filtered through a pad of Celite. The filtrate was concentrated under reduced pressure. The resultant residue was purified by silica gel column chromatography (hexane $/$ EtOAC $=6 / 1$ to $5 / 1$ ) to afford 16 (554 mg, 87\%) as a colorless oil. $[\alpha]_{D}^{24}+39.0\left(\mathrm{C} 0.33, \mathrm{CHCl}_{3}\right)$. IR $v_{\max }$ (neat): 2956, 1732, 1514, $1254,1098 \mathrm{~cm}^{-1}$. ${ }^{1} \mathrm{H}-\mathrm{NMR}\left(600 \mathrm{MHz}, \mathrm{CDCl}_{3}\right) \delta: 7.23(2 \mathrm{H}, \mathrm{d}, J=8.6 \mathrm{~Hz}), 6.88(2 \mathrm{H}, \mathrm{d}, J=8.6 \mathrm{~Hz}), 6.75$ $(1 \mathrm{H}, \mathrm{dd}, J=9.9,4.1 \mathrm{~Hz}), 6.05(1 \mathrm{H}, \mathrm{dd}, J=9.9,0.8 \mathrm{~Hz}), 5.95-5.99(2 \mathrm{H}, \mathrm{m}), 4.87$ $-4.90(1 \mathrm{H}, \mathrm{br} \mathrm{m}), 4.56(1 \mathrm{H}, \mathrm{d}, J=11.6 \mathrm{~Hz}), 4.50(1 \mathrm{H}, \mathrm{d}, J=11.6 \mathrm{~Hz}), 4.13(1 \mathrm{H}$, 
$\mathrm{td}, J=4.1,0.8 \mathrm{~Hz}), 3.92(1 \mathrm{H}, \mathrm{q}, J=6.7 \mathrm{~Hz}), 3.77-3.85(2 \mathrm{H}, \mathrm{m}), 3.81(3 \mathrm{H}, \mathrm{s})$, $3.69(1 \mathrm{H}, \mathrm{dd}, J=8.7,1.1 \mathrm{~Hz}), 3.52(1 \mathrm{H}, t, J=7.8 \mathrm{~Hz}), 1.88(1 \mathrm{H}, \mathrm{ddd}, J=14.0$, $10.3,1.1 \mathrm{~Hz}), 1.37(3 \mathrm{H}, \mathrm{s}), 1.37(3 \mathrm{H}, \mathrm{s}), 1.32(3 \mathrm{H}, \mathrm{s}), 1.08(1 \mathrm{H}, \mathrm{ddd}, J=14.0$, $8.7,1.2 \mathrm{~Hz}), 0.89(9 \mathrm{H}, \mathrm{s}), 0.88(18 \mathrm{H}, \mathrm{s}), 0.12(3 \mathrm{H}, \mathrm{s}), 0.11(6 \mathrm{H}, \mathrm{s}), 0.10(3 \mathrm{H}$, s), $0.08(3 \mathrm{H}, \mathrm{s}), 0.07(3 \mathrm{H}, \mathrm{s}) \cdot{ }^{13} \mathrm{C}-\mathrm{NMR}\left(100 \mathrm{MHz}, \mathrm{CDCl}_{3}\right) \delta_{\mathrm{c}}: 162.8,159.6,144.2,140.7$, $129.4(2 \mathrm{C}), 129.3,122.5,122.4,113.9(2 \mathrm{C}), 109.2,80.2,79.5,77.8,76.8,71.4$, $71.4,69.5,65.8,55.3,36.8,26.5,26.2(3 C), 26.2(3 C), 26.2(3 C), 26.0,25.4$, 18.5, 18.4, 18.3, -1.6, -1.7, -2.8, -3.6, -3.7, -3.8. HR ESIMS m/z 843.4713 $\left(\mathrm{M}+\mathrm{Na}^{+}\right)(\mathrm{calcd}$ for 842.4689$)$.

$\left(5 S, 6 S, 1^{\prime} E, 3^{\prime} R, 4^{\prime} R, 6^{\prime} R, 7^{\prime} R\right)-5,6$-Dihydro-5- (4' ' -methoxybenzyloxy) -6- [3', 4', 6' - tr is (tert-butyldimethylsiloxy) -7', 8'-dihydroxy-3'-methyl-1'-octenyl]-2H-pyran-2-o ne

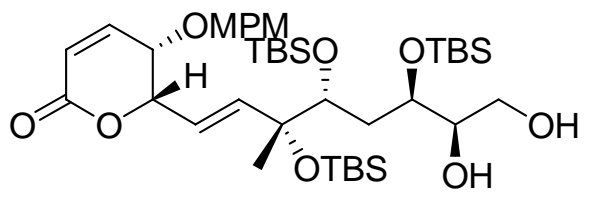

To a stirred solution of $15(4.79 \mathrm{~g}, 5.84 \mathrm{mmol})$ in $\mathrm{MeCN}(120 \mathrm{~mL})$ was added $\mathrm{Zn}\left(\mathrm{NO}_{3}\right)_{2} \cdot 6 \mathrm{H}_{2} \mathrm{O}$ $(34.7 \mathrm{~g}, 117 \mathrm{mmol})$ at $50^{\circ} \mathrm{C}$. The resultant mixture was stirred at the same temperature for $6.5 \mathrm{~h}$. After the addition of saturated $\mathrm{NaHCO}_{3}$ solution, the reaction mixture was extracted with EtOAc. The combined organic layers were washed with $\mathrm{H}_{2} \mathrm{O}$ and saturated $\mathrm{NaCl}$ solution, dried over $\mathrm{Na}_{2} \mathrm{SO}_{4}$, and concentrated under reduced pressure. The resultant residue was purified by silica gel column chromatography (hexane/EtOAc $=4 / 1$ to $1 / 1)$ to afford the diol $(2.99 \mathrm{~g}, 66 \%)$ as a colorless oil and the recovered $15(635 \mathrm{mg}, 13 \%) .[\alpha]_{\mathrm{D}}{ }^{24}+29.8\left(\mathrm{C} 0.41, \mathrm{CHCl}_{3}\right) . \mathrm{IR} v_{\max }$ (neat): 3463, 2930, 1729, 1613, $1514,1253,1096,836 \mathrm{~cm}^{-1} .{ }^{1} \mathrm{H}-\mathrm{NMR}\left(600 \mathrm{MHz}, \mathrm{CDCl}_{3}\right) \delta: 7.26(2 \mathrm{H}, \mathrm{d}, J=8.6 \mathrm{~Hz}), 6.90$ $(2 \mathrm{H}, \mathrm{d}, J=8.6 \mathrm{~Hz}), 6.77(1 \mathrm{H}, \mathrm{dd}, J=9.9,3.4 \mathrm{~Hz}), 6.07(1 \mathrm{H}, \mathrm{dd}, J=9.9,0.8 \mathrm{~Hz})$, $6.03(1 \mathrm{H}, \mathrm{d}, J=15.9 \mathrm{~Hz}), 5.99(1 \mathrm{H}, \mathrm{dd}, J=15.9,5.9 \mathrm{~Hz}), 4.95(1 \mathrm{H}, \mathrm{dd}, J=5.9$, $4.4 \mathrm{~Hz}), 4.60(1 \mathrm{H}, \mathrm{d}, J=11.6 \mathrm{~Hz}), 4.53(1 \mathrm{H}, \mathrm{d}, J=11.6 \mathrm{~Hz}), 4.22$ (1H, br ddd, $J=4.4,3.4,0.8 \mathrm{~Hz}), 3.92(1 \mathrm{H}, \mathrm{td}, J=6.5,3.0 \mathrm{~Hz}), 3.84(3 \mathrm{H}, \mathrm{s}), 3.58-3.67$ $(2 \mathrm{H}, \mathrm{m}), 3.64(1 \mathrm{H}, \mathrm{dd}, J=6.5,5.2 \mathrm{~Hz}), 3.55(1 \mathrm{H}, \mathrm{dd}, J=10.9,7.8 \mathrm{~Hz}), 2.25(1 \mathrm{H}$, $\mathrm{d}, J=7.4 \mathrm{~Hz}, \mathrm{OH}), 2.06(1 \mathrm{H}$, br $\mathrm{d}, J=6.5 \mathrm{~Hz}, \mathrm{OH}), 1.91(1 \mathrm{H}, \mathrm{ddd}, J=14.4,6.5$, $5.2 \mathrm{~Hz}), 1.66(1 \mathrm{H}, \mathrm{dt}, 14.4,6.5 \mathrm{~Hz}), 1.42(3 \mathrm{H}, \mathrm{s}), 0.92(9 \mathrm{H}, \mathrm{s}), 0.91(18 \mathrm{H}, \mathrm{s})$, $0.16(3 \mathrm{H}, \mathrm{s}), 0.14(6 \mathrm{H}, \mathrm{s}), 0.12(3 \mathrm{H}, \mathrm{s}), 0.12(3 \mathrm{H}, \mathrm{s}), 0.11(3 \mathrm{H}, \mathrm{s}) .{ }^{13} \mathrm{C}-\mathrm{NMR} \quad(100$ $\left.\mathrm{MHz}, \mathrm{CDCl}_{3}\right) \delta_{\mathrm{C}}: 162.8,159.6,144.3,140.3,129.5(2 \mathrm{C}), 129.3,122.9,122.4,114.0$ $(2 \mathrm{C}), 80.0,78.5,76.9,73.8,71.4,70.7,69.4,64.4,55.3,37.5,26.5,26.3(3 \mathrm{C})$, $26.1(3 \mathrm{C}), 25.9(3 \mathrm{C}), 18.5,18.2,18.1,-1.5,-1.5,-2.9,-3.4,-4.0,-4.3$. HR ESIMS $\mathrm{m} / \mathrm{z} 803.4397\left(\mathrm{M}+\mathrm{Na}^{+}\right)(\mathrm{calcd}$ for 803.4376$)$. 
$\left(5 S, 6 S, 1^{\prime} E, 3^{\prime} R, 4^{\prime} R, 6^{\prime} R, 7^{\prime} E Z\right)-5,6$-Dihydro- (4' '-methoxybenzyloxy) -6- [3', 4' , 6'-tri s (tert-butyldimethylsiloxy)-8'-iodo-3'-methyl-1', 7'-octadienyl]-2H-pyran-2-one (17)

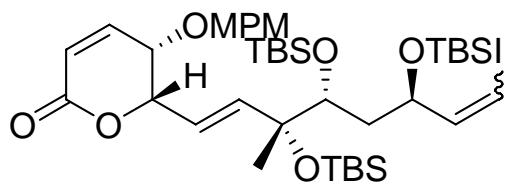

To a stirred solution of the diol (2.98 $\mathrm{g}, 3.81 \mathrm{mmol})$ in $\mathrm{THF}$ and $\mathrm{H}_{2} \mathrm{O}(4: 1,38 \mathrm{~mL})$ was added $\mathrm{NaIO}_{4}(2.44 \mathrm{~g}, 11.4 \mathrm{mmol})$ at room temperature. After stirred $30 \mathrm{~min}$, the reaction mixture was filtered through a pad of Celite, and extracted with EtoAc. The combined organic layers were washed with $\mathrm{H}_{2} \mathrm{O}$ and saturated $\mathrm{NaCl}$ solution, dried over $\mathrm{Na}_{2} \mathrm{SO}_{4}$, and concentrated under reduced pressure. The crude aldehyde was used directly to the next step.

To a stirred solution of iodomethyltriphenylphosphonium iodide (2.26 g, $4.19 \mathrm{mmol})$ in THF (19 mL) was added NaHMDS (1.0 M in THF, $4.2 \mathrm{~mL}, 4.2 \mathrm{mmol}$ ) at room temperature. The mixture was stirred for $15 \mathrm{~min}$ at the same temperature, then cooled to $-78{ }^{\circ} \mathrm{C}$ followed by addition of HMPA $(3.3 \mathrm{~mL}, 19.0 \mathrm{mmol})$. After the dropwise addition of the crude aldehyde above in THF (19 mL) at $-100^{\circ} \mathrm{C}$, the reaction mixture was allowed to warm to $0{ }^{\circ} \mathrm{C}$ and the reaction was quenched by the addition of $\mathrm{NH}_{4} \mathrm{Cl}$ solution. The reaction mixture was extracted with EtOAc. The combined organic layers were washed with $\mathrm{H}_{2} \mathrm{O}$ and saturated $\mathrm{NaCl}$ solution, dried over $\mathrm{Na}_{2} \mathrm{SO}_{4}$, and concentrated under reduced pressure. The resultant residue was purified by silica gel column chromatography (hexane/EtOAC $=9 / 1)$ to afford $17(Z: E=2: 1,1.52 \mathrm{~g}, 77 \%)$ as a colorless oil. $I R v_{\max }$ (neat) : 2956, 1732, 1612, 1514, 1253, 1094, $836 \mathrm{~cm}^{-1} .{ }^{1} \mathrm{H}-\mathrm{NMR}\left(600 \mathrm{MHz}, \mathrm{CDCl}_{3}\right)$ $Z$-isomer $\delta: 7.21(2 \mathrm{H}, \mathrm{d}, J=8.5 \mathrm{~Hz}), 6.87(2 \mathrm{H}, \mathrm{d}, J=8.5 \mathrm{~Hz}), 6.67(1 \mathrm{H}, \mathrm{dd}, J=$ $9.9,4.1 \mathrm{~Hz}), 6.17(1 \mathrm{H}, \mathrm{t}, J=7.6 \mathrm{~Hz}), 6.12(1 \mathrm{H}, \mathrm{d}, J=7.6 \mathrm{~Hz}), 6.05(1 \mathrm{H}, \mathrm{dd}$, $J=15.8,8.0 \mathrm{~Hz}), 6.04(1 \mathrm{H}, \mathrm{d}, J=9.9 \mathrm{~Hz}), 6.00(1 \mathrm{H}, \mathrm{d}, J=15.8 \mathrm{~Hz}), 4.88(1 \mathrm{H}$, $\mathrm{d}, J=8.0,4.1 \mathrm{~Hz}), 4.57(1 \mathrm{H}, \mathrm{d}, J=11.7 \mathrm{~Hz}), 4.47(1 \mathrm{H}, \mathrm{d}, J=11.5,7.6 \mathrm{~Hz}), 4.42$ $(1 \mathrm{H}, \mathrm{d}, J=11.7 \mathrm{~Hz}), 4.02(1 \mathrm{H}, \mathrm{t}, J=4.1 \mathrm{~Hz}), 3.82(3 \mathrm{H}, \mathrm{s}), 3.78(1 \mathrm{H}, \mathrm{d}, J=9.0$ $\mathrm{Hz}), 2.08(1 \mathrm{H}, \mathrm{dd}, J=13.6,11.5 \mathrm{~Hz}), 1.40(3 \mathrm{H}, \mathrm{s}), 1.12(1 \mathrm{H}, \mathrm{ddd}, J=11.3,9.0$ $\mathrm{Hz}), 0.93(9 \mathrm{H}, \mathrm{s}), 0.91(9 \mathrm{H}, \mathrm{s}), 0.88(9 \mathrm{H}, \mathrm{s}), 0.27(3 \mathrm{H}, \mathrm{s}), 0.12(3 \mathrm{H}, \mathrm{s}), 0.11(6 \mathrm{H}$, s), $0.10(3 \mathrm{H}, \mathrm{s}), 0.04(3 \mathrm{H}, \mathrm{s})$. E-isomer $\delta: 7.23(2 \mathrm{H}, \mathrm{d}, J=8.5 \mathrm{~Hz}), 6.90(2 \mathrm{H}, \mathrm{d}$, $J=8.5 \mathrm{~Hz}), 6.71(1 \mathrm{H}, \mathrm{d}, J=9.9,4.0 \mathrm{~Hz}), 6.45(1 \mathrm{H}, \mathrm{dd}, J=14.4,8.3 \mathrm{~Hz}), 6.21$ $(1 \mathrm{H}, \mathrm{d}, \mathrm{J}=14.4 \mathrm{~Hz}), 6.10-6.18(1 \mathrm{H}, \mathrm{m}), 5.96-6.06(2 \mathrm{H}, \mathrm{m}), 4.89(1 \mathrm{H}, \mathrm{dd}, \mathrm{J}=$ $8.4,4.0 \mathrm{~Hz}), 4.59(1 \mathrm{H}, \mathrm{d}, J=11.8 \mathrm{~Hz}), 4.45(1 \mathrm{H}, \mathrm{d}, J=11.8 \mathrm{~Hz}), 4.22(1 \mathrm{H}, \mathrm{td}$, $J=8.3,2.2 \mathrm{~Hz}), 4.07(1 \mathrm{H}, \mathrm{t}, J=4.0 \mathrm{~Hz}), 3.82(3 \mathrm{H} . \mathrm{s}), 3.67(1 \mathrm{H}, \mathrm{dd}, J=8.3$, $2.0 \mathrm{~Hz}), 2.05-2.10(1 \mathrm{H}, \mathrm{m}), 1.38(3 \mathrm{H}, \mathrm{s}), 1.19(1 \mathrm{H}, \mathrm{d}, \mathrm{J}=13.3,8.3,2.2 \mathrm{~Hz})$, $0.90(18 \mathrm{H}, \mathrm{s}), 0.87(9 \mathrm{H}, \mathrm{s}), 0.01-0.28(9 \mathrm{H}, \mathrm{m})$. HR ESIMS m/z $895.3291\left(\mathrm{M}+\mathrm{Na}^{+}\right)(\mathrm{calcd}$ 
$\left(5 S, 6 S, 1^{\prime} E, 3^{\prime} R, 4^{\prime} R, 6^{\prime} R, 7^{\prime} E Z\right)-5,6$-Dihydro-5-(4' ' -methoxybenzyloxy) -6- (3', 4', 6'-t rihydroxy-8'-iodo-3'-methyl-1', 7'-octadienyl)-2H-pyran-2-one (18)<smiles>CO[C@H]1C=CC(=O)O[C@H]1/C=C/[C@@](C)(O)[C@@H](O)C[C@H](O)/C=C\I</smiles>

The reaction was performed in a teflon vessel. To a stirred solution of 17 (326 mg, $0.373 \mathrm{mmol}$ ) in MeCN (3.7 mL) was added $\mathrm{Et}_{3} \mathrm{~N} \cdot 3 \mathrm{HF}$ (1.82 mL, $\left.11.2 \mathrm{mmol}\right)$, followed by $\mathrm{Et}_{3} \mathrm{~N}(780 \mu \mathrm{L}, 5.60 \mathrm{mmol})$. The resultant mixture was stirred at $60{ }^{\circ} \mathrm{C}$ for $29 \mathrm{~h}$. After the addition of saturated $\mathrm{NaHCO}_{3}$ solution, the reaction mixture was extracted with EtOAC. The combined organic layers were washed with $\mathrm{H}_{2} \mathrm{O}$ and saturated $\mathrm{NaCl}$ solution, dried over $\mathrm{Na}_{2} \mathrm{SO}_{4}$, and concentrated under reduced pressure. The resultant residue was purified by silica gel column chromatography (EtOAc only) to afford 18 (198 mg, $100 \%)$ as a white amorphous solid. IR $v_{\max }(\mathrm{KBr}): 3423,3012,1718,1514,1252,1048$, $826 \mathrm{~cm}^{-1} .{ }^{1} \mathrm{H}$-NMR $\left(600 \mathrm{MHz}, \mathrm{CDCl}_{3}\right) \quad z$-isomer $\delta: 7.25(2 \mathrm{H}, \mathrm{d}, J=8.5 \mathrm{~Hz}), 6.90(2 \mathrm{H}, \mathrm{d}$, $J=8.5 \mathrm{~Hz}), 6.83(1 \mathrm{H}, \mathrm{dd}, J=9.9,4.2 \mathrm{~Hz}), 6.37(1 \mathrm{H}, t, J=7.5 \mathrm{~Hz}), 6.32(1 \mathrm{H}$, $\mathrm{d}, J=7.5 \mathrm{~Hz}), 6.11(1 \mathrm{H}, \mathrm{d}, J=9.9 \mathrm{~Hz}), 6.03(1 \mathrm{H}, \mathrm{dd}, J=15.4,6.0 \mathrm{~Hz}), 5.95(1 \mathrm{H}$, $\mathrm{d}, J=15.4 \mathrm{~Hz}), 4.94-4.97(1 \mathrm{H}, \mathrm{m}), 4.62-4.68(1 \mathrm{H}, \mathrm{m}), 4.56(1 \mathrm{H}, \mathrm{d}, J=11.5$ $\mathrm{Hz}), 4.53(1 \mathrm{H}, \mathrm{d}, J=11.5 \mathrm{~Hz}), 4.12-4.16(1 \mathrm{H}, \mathrm{m}), 3.82(3 \mathrm{H}, \mathrm{s}), 3.70-3.80(1 \mathrm{H}$, $\mathrm{m}), 2.90(2 \mathrm{H}, \mathrm{br} \mathrm{m}, \mathrm{OH}), 2.41(1 \mathrm{H}, \mathrm{br} \mathrm{s}, \mathrm{OH}), 1.62-1.83(2 \mathrm{H}, \mathrm{m}), 1.28(3 \mathrm{H}, \mathrm{s})$. E-isomer $\delta: 7.24(2 \mathrm{H}, \mathrm{d}, J=8.5 \mathrm{~Hz}), 6.90(2 \mathrm{H}, \mathrm{d}, J=8.5 \mathrm{~Hz}), 6.84(1 \mathrm{H}, \mathrm{dd}, J=$ $9.9,4.7 \mathrm{~Hz}), 6.58(1 \mathrm{H}, \mathrm{dd}, J=14.3,5.3 \mathrm{~Hz}), 6.38(1 \mathrm{H}, \mathrm{d}, J=14.3 \mathrm{~Hz}), 6.12(1 \mathrm{H}$, $\mathrm{d}, J=9.9 \mathrm{~Hz}), 5.93-6.05(2 \mathrm{H}, \mathrm{m}), 4.94-4.97(1 \mathrm{H}, \mathrm{m}), 4.30-4.40(1 \mathrm{H}, \mathrm{m}), 4.56$ $(1 \mathrm{H}, \mathrm{d}, J=11.4 \mathrm{~Hz}), 4.53(1 \mathrm{H}, \mathrm{d}, J=11.4 \mathrm{~Hz}), 4.08-4.11(1 \mathrm{H}, \mathrm{m}), 3.82(3 \mathrm{H}, \mathrm{s})$, $3.56-3.58(1 \mathrm{H}, \mathrm{s}), 2.96(1 \mathrm{H}, \mathrm{br} \mathrm{s}, \mathrm{OH}), 2.86(1 \mathrm{H}, \mathrm{br} \mathrm{s}, \mathrm{OH}), 2.32(1 \mathrm{H}, \mathrm{br} \mathrm{s}, \mathrm{OH})$, $1.62-1.83(2 \mathrm{H}, \mathrm{m}), 1.26(3 \mathrm{H}, \mathrm{s})$. HR ESIMS m/z $553.0692\left(\mathrm{M}+\mathrm{Na}^{+}\right)$(calcd for 553.0693$)$.

$\left(5 S, 6 S, 1^{\prime} E, 3^{\prime} R, 4^{\prime} R, 6^{\prime} R, 7^{\prime} E Z\right)-6-\left[6^{\prime}\right.$ - tert-Butyldimethylsiloxy-8'-iodo-3'-methyl-3 ' ,4'-bis (triethylsiloxy)-1', 7'-octadienyl] -5,6-dihydro-5- (4' '-methoxybenzyloxy) -2H-pyran-2-one (19)

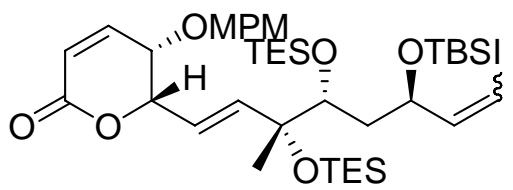

To a stirred solution of $18\left(296 \mathrm{mg}, 0.56 \mathrm{mmol}\right.$ ) in $\mathrm{CH}_{2} \mathrm{Cl}_{2}$ (11 mL) was added 2,6-lutidine $(510 \mu \mathrm{l}, 4.4 \mathrm{mmol})$, followed by TBSOTf $(140 \mu \mathrm{l}, 0.62 \mathrm{mmol})$ at $-78{ }^{\circ} \mathrm{C}$, and the mixture has stirred at $-78{ }^{\circ} \mathrm{C}$. After the addition of TESOTf $(510 \mu \mathrm{l}, 2.24 \mathrm{mmol})$ at the same temperature, the mixture was stirred at the same temperature for $1 \mathrm{~h}$. Then saturated 
$\mathrm{NaHCO}_{3}$ solution was added to the mixture. The reaction mixture was extracted with EtOAC and the combined organic layers were washed with $\mathrm{H}_{2} \mathrm{O}$ and saturated $\mathrm{NaCl}$ solution, dried over $\mathrm{Na}_{2} \mathrm{SO}_{4}$, and concentrated under reduced pressure. The resultant residue was purified by silica gel column chromatography (hexane/EtOAc = 9/1 to 8/1) to afford 19 (435 mg, 89\%) as a colorless oil. IR $v_{\max }$ (neat): 2954, 1735, 1612, 1250, 1095, $834 \mathrm{~cm}^{-1} \cdot{ }^{1} \mathrm{H}-\mathrm{NMR}\left(600 \mathrm{MHz}, \mathrm{CDCl}_{3}\right)$ z-isomer $\delta: 7.22(2 \mathrm{H}, \mathrm{d}, J=8.6 \mathrm{~Hz}), 6.90(2 \mathrm{H}, \mathrm{d}$, $J=8.6 \mathrm{~Hz}), 6.72(1 \mathrm{H}, \mathrm{dd}, J=9.9,4.6 \mathrm{~Hz}), 6.08-6.16(2 \mathrm{H}, \mathrm{m}), 6.06(1 \mathrm{H}, \mathrm{d}, \mathrm{J}$ $=9.9 \mathrm{~Hz}), 6.02(1 \mathrm{H}, \mathrm{dd}, J=15.7,6.8 \mathrm{~Hz}), 5.97(1 \mathrm{H}, \mathrm{d}, J=15.7 \mathrm{~Hz}), 4.89(1 \mathrm{H}$, $\mathrm{dd}, J=6.8,4.0 \mathrm{~Hz}), 4.59(1 \mathrm{H}, \mathrm{d}, J=11.6 \mathrm{~Hz}), 4.56(1 \mathrm{H}, \mathrm{d}, J=11.6 \mathrm{~Hz}), 4.40$ $-4.48(1 \mathrm{H}, \mathrm{m}), 4.02(1 \mathrm{H}, \mathrm{t}, J=4.0 \mathrm{~Hz}), 3.82(3 \mathrm{H}, \mathrm{s}), 3.77(1 \mathrm{H}, \mathrm{d}, J=8.8 \mathrm{~Hz})$, $1.95(1 \mathrm{H}, \mathrm{dd}, J=13.9,11.5 \mathrm{~Hz}), 1.38(3 \mathrm{H}, \mathrm{s}), 1.10(1 \mathrm{H}, \mathrm{ddd}, J=13.9,8.8,2.4$ $\mathrm{Hz}), 1.01(9 \mathrm{H}, t, J=8.0 \mathrm{~Hz}), 0.95(9 \mathrm{H}, t, J=8.0 \mathrm{~Hz}), 0.88(9 \mathrm{H}, \mathrm{s}), 0.62(6 \mathrm{H}$, $q, J=8.0 \mathrm{~Hz}), 0.61(6 \mathrm{H}, \mathrm{q}, J=8.0 \mathrm{~Hz}), 0.12(3 \mathrm{H}, \mathrm{s}), 0.05(3 \mathrm{H}, \mathrm{s})$. E-isomer $\delta$ : $7.24(1 \mathrm{H}, \mathrm{d}, J=8.6 \mathrm{~Hz}), 6.90(1 \mathrm{H}, \mathrm{d}, J=8.6 \mathrm{~Hz}), 6.72-6.78(1 \mathrm{H}, \mathrm{m}), 6.48(1 \mathrm{H}$, $J=14.5,8.6 \mathrm{~Hz}), 6.06-6.08(1 \mathrm{H}, \mathrm{m}), 6.08-6.22(1 \mathrm{H}, \mathrm{m}), 5.88-6.04(2 \mathrm{H}, \mathrm{m})$, $4.91(1 \mathrm{H}, \mathrm{dd}, J=5.9,4.0 \mathrm{~Hz}), 4.48(1 \mathrm{H}, \mathrm{d}, J=11.5 \mathrm{~Hz}), 4.45(1 \mathrm{H}, \mathrm{d}, J=11.5$ $\mathrm{Hz}), 4.19(1 \mathrm{H}, \mathrm{td}, J=8.6,3.9 \mathrm{~Hz}), 4.08(1 \mathrm{H}, t, J=4.0 \mathrm{~Hz}), 3.82(3 \mathrm{H}, \mathrm{s}), 3.65$ $(1 \mathrm{H}, \mathrm{dd}, J=7.9,3.1 \mathrm{~Hz}), 1.96-2.00(1 \mathrm{H}, \mathrm{m}), 1.37(3 \mathrm{H}, \mathrm{s}), 1.20(1 \mathrm{H}, J=14.0$, $7.9,3.9 \mathrm{~Hz}), 0.93-1.04(18 \mathrm{H}, \mathrm{m}), 0.87(9 \mathrm{H}, \mathrm{s}), 0.65-0.75(12 \mathrm{H}, \mathrm{m}), 0.05(3 \mathrm{H}$, s), $0.03(3 \mathrm{H}, \mathrm{s})$. HR ESIMS m/z $895.3289\left(\mathrm{M}+\mathrm{Na}^{+}\right)$(calcd for 895.3288).

$\left(5 S, 6 S, 1^{\prime} E, 3^{\prime} R, 4^{\prime} R, 6^{\prime} R, 7^{\prime} E Z\right)-6$ - [6'-tert-Butyldimethylsiloxy-8' -iodo-3'-methyl-3 ', 4'-bis (triethylsiloxy)-1', 7'-octadienyl] -5,6-dihydro-5-hydroxy-2H-pyran-2-one

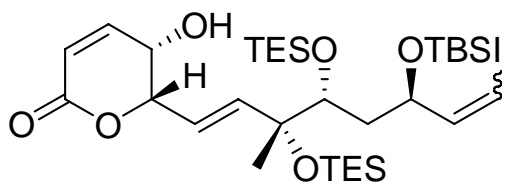

To a stirred solution of $19\left(405 \mathrm{mg}, 0.465 \mathrm{mmol}\right.$ ) in $\mathrm{CH}_{2} \mathrm{Cl}_{2}$ (9 mL) and $\mathrm{H}_{2} \mathrm{O}$ ( $1 \mathrm{~mL}$ ) was added DDQ (1.05 g, $4.63 \mathrm{mmol})$ at room temperature, and the resultant mixture was stirred at room temperature for $2 \mathrm{~h}$. After the addition of saturated $\mathrm{NaHCO}_{3}$ solution, the reaction mixture was extracted with EtOAc. The combined organic layers were washed with saturated $\mathrm{NaHCO}_{3}$ solution, $\mathrm{H}_{2} \mathrm{O}$ and saturated $\mathrm{NaCl}$ solution, dried over $\mathrm{Na}_{2} \mathrm{SO}_{4}$, and concentrated under reduced pressure. The resultant residue was purified by silica gel column chromatography (hexane/EtOA = 9/1 to 4/1) to afford the alcohol (297 mg, $84 \%$ ) as a colorless oil. IR $v_{\max }$ (neat) : 3441, 2955, 1731, 1554, 1092, $834 \mathrm{~cm}^{-1} \cdot{ }^{1} \mathrm{H}-\mathrm{NMR}$ $\left(600 \mathrm{MHz}, \mathrm{CDCl}_{3}\right) \quad z$-isomer $\delta: 6.99(1 \mathrm{H}, \mathrm{dd}, J=9.7,5.6 \mathrm{~Hz}), 6.21(1 \mathrm{H}, \mathrm{d}, J=7.8$ $\mathrm{Hz}), 6.18(1 \mathrm{H}, \mathrm{t}, J=7.8 \mathrm{~Hz}), 6.15(1 \mathrm{H}, \mathrm{d}, J=9.7 \mathrm{~Hz}), 6.09(1 \mathrm{H}, \mathrm{dd}, J=15.9$, $1.3 \mathrm{~Hz}), 5.67(1 \mathrm{H}, \mathrm{dd}, J=15.9,5.7 \mathrm{~Hz}), 4.94(1 \mathrm{H}, \mathrm{ddd}, J=5.7,3.0,1.3 \mathrm{~Hz}), 4.43$ $(1 \mathrm{H}, \mathrm{ddd}, J=10.3,7.8,2.9 \mathrm{~Hz}), 4.13(1 \mathrm{H}, \mathrm{td}, J=5.6,3.0 \mathrm{~Hz}), 3.76(1 \mathrm{H}, \mathrm{dd}, J$ 
$=8.2,1.4 \mathrm{~Hz}), 2.27(1 \mathrm{H}$, br $d, J=5.6 \mathrm{~Hz}, \mathrm{OH}), 2.04(1 \mathrm{H}, \mathrm{ddd}, J=14.2,10.3,1.4$ $\mathrm{Hz}), 1.38(3 \mathrm{H}, \mathrm{s}), 1.13(1 \mathrm{H}, \mathrm{ddd}, J=14.2,8.2,2.9 \mathrm{~Hz}), 1.00(9 \mathrm{H}, t, J=8.0 \mathrm{~Hz})$, $0.96(9 \mathrm{H}, t, J=8.0 \mathrm{~Hz}), 0.88(9 \mathrm{H}, \mathrm{s}), 0.72(6 \mathrm{H}, \mathrm{q}, J=8.0 \mathrm{~Hz}), 0.62(6 \mathrm{H}, \mathrm{q}, J$ $=8.0 \mathrm{~Hz}), 0.13(3 \mathrm{H}, \mathrm{s}), 0.06(3 \mathrm{H}, \mathrm{s})$. E-isomer $\delta: 7.00(1 \mathrm{H}, \mathrm{dd}, J=9.7,5.5 \mathrm{~Hz})$, $6.53(1 \mathrm{H}, \mathrm{dd}, J=14.5,8.1 \mathrm{~Hz}), 6.22(1 \mathrm{H}, \mathrm{d}, J=14.5 \mathrm{~Hz}), 6.16(1 \mathrm{H}, \mathrm{d}, J=9.7$ $\mathrm{Hz}), 6.10(1 \mathrm{H}, \mathrm{dd}, J=15.9,1.1 \mathrm{~Hz}), 5.67(1 \mathrm{H}, \mathrm{dd}, J=15.9,5.3 \mathrm{~Hz}), 4.95(1 \mathrm{H}$, $\mathrm{ddd}, J=5.3,2.6,1.1 \mathrm{~Hz}), 4.21(1 \mathrm{H}, \mathrm{td}, J=8.1,4.7 \mathrm{~Hz}), 4.10(1 \mathrm{H}, \mathrm{td}, J=5.5$, $2.6 \mathrm{~Hz}), 3.63(1 \mathrm{H}, \mathrm{dd}, J=7.1,3.9 \mathrm{~Hz}), 2.24(1 \mathrm{H}, \mathrm{d}, J=5.5 \mathrm{~Hz}), 2.03$ (1H, ddd, $J=14.0,8.1,4.0 \mathrm{~Hz}), 1.40(3 \mathrm{H}, \mathrm{s}), 1.21(1 \mathrm{H}, J=14.0,7.0,4.7 \mathrm{~Hz}), 0.97(9 \mathrm{H}$, $t, J=7.9 \mathrm{~Hz}), 0.96(9 \mathrm{H}, \mathrm{t}, J=7.9 \mathrm{~Hz}), 0.87(9 \mathrm{H}, \mathrm{s}), 0.64(6 \mathrm{H}, \mathrm{q}, J=7.9 \mathrm{~Hz})$, $0.62(6 \mathrm{H}, \mathrm{q}, J=7.9 \mathrm{~Hz}), 0.05(3 \mathrm{H}, \mathrm{s}), 0.04(3 \mathrm{H}, \mathrm{s})$. HR ESIMS m/z $775.2730\left(\mathrm{M}+\mathrm{Na}^{+}\right)(\mathrm{calcd}$ for 775.2718$)$.

$\left(5 S, 6 S, 1^{\prime} E, 3^{\prime} R, 4^{\prime} R, 6^{\prime} R, 7^{\prime} E Z\right)$-5-tert-Butyldimethylsiloxy-6- [6' - tert-butyldimethy lsiloxy-8' -iodo-3'-methyl-3' , 4' -bis (triethylsiloxy)-1', 7' -octadienyl] -5,6-dihyd ro-2H-pyran-2-one

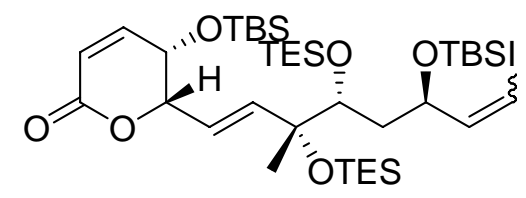

To a stirred solution of the alcohol (240 mg, $0.318 \mathrm{mmol}$ ) in $\mathrm{CH}_{2} \mathrm{Cl}_{2}$ (1.5 mL) were

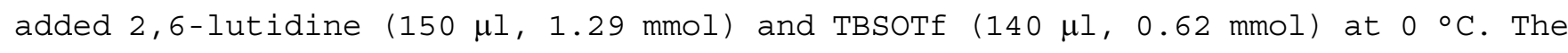
resultant mixture was stirred at the same temperature for $30 \mathrm{~min}$. After the addition of $\mathrm{NaHCO}_{3}$ solution, the reaction mixture was extracted with EtOAC. The combined organic layers were washed with $\mathrm{H}_{2} \mathrm{O}$ and saturated $\mathrm{NaCl}$ solution, dried over $\mathrm{Na}_{2} \mathrm{SO}_{4}$, and concentrated under reduced pressure. The resultant residue was purified by silica gel column chromatography (hexane/EtOAC $=30 / 1$ to 20/1) to afford the TBS ether (274 mg, 99\%) as a colorless oil. IR $v_{\max }$ (neat): 2955, 1739, 1252, $1103 \mathrm{~cm}^{-1} \cdot{ }^{1} \mathrm{H}-\mathrm{NMR}$ (600 $\left.\mathrm{MHz}, \mathrm{CDCl}_{3}\right) z$-isomer $\delta: 6.78(1 \mathrm{H}, \mathrm{dd}, J=9.8,4.1 \mathrm{~Hz}), 6.13-6.18(2 \mathrm{H}, \mathrm{m}), 6.03$ $(1 \mathrm{H}, \mathrm{d}, J=9.8 \mathrm{~Hz}), 5.90-5.96(2 \mathrm{H}, \mathrm{m}), 4.78(1 \mathrm{H}, \mathrm{dd}, J=5.8,4.1 \mathrm{~Hz}), 4.40-$ $4.47(1 \mathrm{H}, \mathrm{m}), 4.30(1 \mathrm{H}, \mathrm{t}, J=4.1 \mathrm{~Hz}), 3.76(1 \mathrm{H}, \mathrm{d}, J=9.7 \mathrm{~Hz}), 1.93(1 \mathrm{H}, \mathrm{dd}, J$ $=13.5,11.0 \mathrm{~Hz}), 1.36(3 \mathrm{H}, \mathrm{s}), 1.05(1 \mathrm{H}, \mathrm{dd}, J=13.5,9.7 \mathrm{~Hz}), 1.00(9 \mathrm{H}, t, J=$ $8.0 \mathrm{~Hz}), 0.95(9 \mathrm{H}, t, J=8.0 \mathrm{~Hz}), 0.89(9 \mathrm{H}, \mathrm{s}), 0.88(9 \mathrm{H}, \mathrm{s}), 0.72(6 \mathrm{H}, q, J=8.0$ $\mathrm{Hz}), 0.61(6 \mathrm{H}, \mathrm{q}, J=8.0 \mathrm{~Hz}), 0.12(3 \mathrm{H}, \mathrm{s}), 0.08(3 \mathrm{H}, \mathrm{s}), 0.06(3 \mathrm{H}, \mathrm{s}), 0.04(3 \mathrm{H}$, s). E-isomer $\delta: 6.75-6.78(1 \mathrm{H}, \mathrm{m}), 6.47(1 \mathrm{H}, \mathrm{ddd}, J=14.4,8.7 \mathrm{~Hz}), 6.18-6.23$ $(1 \mathrm{H}, \mathrm{m}), 5.99-6.03(1 \mathrm{H}, \mathrm{m}), 5.90-5.97(2 \mathrm{H}, \mathrm{m}), 4.79(1 \mathrm{H}, \mathrm{dd}, \mathrm{J}=7.8,3.8 \mathrm{~Hz})$, $4.38(1 \mathrm{H}, t, J=3.8 \mathrm{~Hz}), 4.17(1 \mathrm{H}, \mathrm{td}, J=8.7,3.2 \mathrm{~Hz}), 3.65(1 \mathrm{H}, \mathrm{dd}, J=8.6$, $1.5 \mathrm{~Hz}), 1.93-1.98(1 \mathrm{H}, \mathrm{m}), 1.35(3 \mathrm{H}, \mathrm{s}), 1.13(1 \mathrm{H}, \mathrm{ddd}, J=12.7,8.6,3.2 \mathrm{~Hz})$, $0.86-1.02(36 \mathrm{H}, \mathrm{m}), 0.56-0.75(12 \mathrm{H}, \mathrm{m}), 0.04-0.12(12 \mathrm{H}, \mathrm{m})$. HR ESIMS m/z 889.3563 
$\left(\mathrm{M}+\mathrm{Na}^{+}\right)(\mathrm{calcd}$ for 889.3577$)$.

$\left(5 S, 6 S, 1^{\prime} E, 3^{\prime} R, 4^{\prime} R, 6^{\prime} R, 7^{\prime} Z\right)-5$-tert-Butyldimethylsiloxy-6- [6' - tert-butyldimethyl siloxy-4' -hydroxy-8'-iodo-3'-methyl-3' , 4' -bis (triethylsiloxy) -1', 7'-octadienyl] $-5,6$-dihydro-2H-pyran-2-one $(20)$

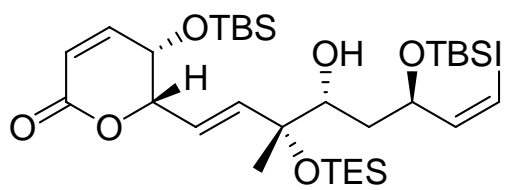

To a stirred solution of the TES ether (186 mg, $0.214 \mathrm{mmol}$ ) in THF (4.3 $\mathrm{mL}$ ) and MeCN $(8.6 \mathrm{~mL})$ was added $1 \mathrm{M} \mathrm{HCl}(1.4 \mathrm{~mL})$ at $-10^{\circ} \mathrm{C}$. The resultant mixture was stirred at the same temperature for $4 \mathrm{~h}$. After the addition of $\mathrm{NaHCO}_{3}$ solution, the reaction mixture was extracted with EtOAc. The combined organic layers were washed with $\mathrm{H}_{2} \mathrm{O}$ and saturated $\mathrm{NaCl}$ solution, dried over $\mathrm{Na}_{2} \mathrm{SO}_{4}$, and concentrated under reduced pressure. The resultant residue was purified by silica gel column chromatography (hexane/EtOAC $=20 / 1$ to $8 / 1$ ) to afford 20 (56 mg, 37\%) as a colorless oil. $[\alpha]_{D}^{24}+23.3$ (c 0.14 , $\left.\mathrm{CHCl}_{3}\right)$. IR $v_{\max }$ (neat): 2954, 1731, $1086 \mathrm{~cm}^{-1} \cdot{ }^{1} \mathrm{H}-\mathrm{NMR}\left(600 \mathrm{MHz}, \mathrm{CDCl}_{3}\right) \delta: 6.79$ (1H, dd, $J=9.8,4.0 \mathrm{~Hz}), 6.35(1 \mathrm{H}, \mathrm{t}, J=7.6 \mathrm{~Hz}), 6.23(1 \mathrm{H}, \mathrm{dd}, J=7.6,0.8 \mathrm{~Hz}), 6.04$ $(1 \mathrm{H}, \mathrm{d}, J=9.8 \mathrm{~Hz}), 5.97(1 \mathrm{H}, \mathrm{dd}, J=15.8,6.2 \mathrm{~Hz}), 5.93(1 \mathrm{H}, \mathrm{d}, J=15.8 \mathrm{~Hz})$, $4.82(1 \mathrm{H}, \mathrm{dd}, J=6.2,4.0 \mathrm{~Hz}), 4.66(1 \mathrm{H}, \mathrm{td}, J=7.0,3.2,0.8 \mathrm{~Hz}), 4.35(1 \mathrm{H}, t$, $J=4.0 \mathrm{~Hz}), 3.65(1 \mathrm{H}, \mathrm{ddd}, J=11.0,2.6,1.2 \mathrm{~Hz}), 3.07(1 \mathrm{H}, \mathrm{d}, J=2.6 \mathrm{~Hz}, \mathrm{OH})$, $1.77(1 \mathrm{H}, \mathrm{ddd}, J=14.0,7.0,1.2 \mathrm{~Hz}), 1.37(1 \mathrm{H}, \mathrm{ddd}, J=14.0,11.0,3.2 \mathrm{~Hz}), 1.25$ $(3 \mathrm{H}, \mathrm{s}), 0.93(9 \mathrm{H}, \mathrm{t}, J=8.0 \mathrm{~Hz}), 0.90(9 \mathrm{H}, \mathrm{s}), 0.88(9 \mathrm{H}, \mathrm{s}), 0.58(6 \mathrm{H}, q, J=8.0$ $\mathrm{Hz}), 1.12(3 \mathrm{H}, \mathrm{s}), 0.11(3 \mathrm{H}, \mathrm{s}), 0.10(3 \mathrm{H}, \mathrm{s}), 0.06(3 \mathrm{H}, \mathrm{s}) .{ }^{13} \mathrm{C}-\mathrm{NMR}\left(100 \mathrm{MHz}, \mathrm{CDCl}_{3}\right)$ $\delta_{c}: 163.0,145.7,144.0,140.1,123.4,121.8,81.3,79.9,74.9,74.3,64.5,37.0$, $29.7,25.8(3 \mathrm{C}), 25.7(3 \mathrm{C}), 23.0,18.0,18.0,7.1(3 \mathrm{C}), 6.8(3 \mathrm{C}),-4.2,-4.4,-4.6$, -5.0. HR ESIMS m/z $775.2730\left(\mathrm{M}+\mathrm{Na}^{+}\right)(\mathrm{calcd}$ for 775.2712$)$.

$\left(5 S, 6 S, 1^{\prime} E, 3^{\prime} R, 4^{\prime} R, 6^{\prime} R, 7^{\prime} Z, 9^{\prime} Z, 1^{\prime} E\right)$-5-tert-Butyldimethylsiloxy-6- [6' - tert-buty ldimethylsiloxy-13' - tert-butyldiphenylsiloxy-4' -hydroxy-3'-methyl-3'-triethylsi loxy-1',7', 9',11'-tridecatetraenyl] -5,6-dihydro-2 $H$-pyran-2-one

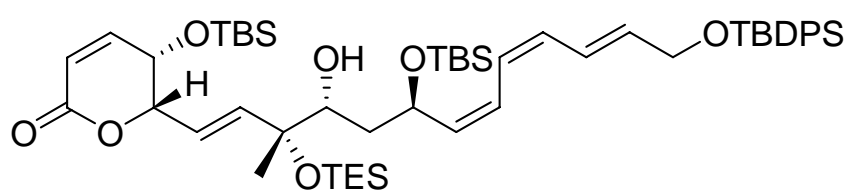

To a stirred solution of 20 (75 mg, $0.100 \mathrm{mmol})$ and stannane $6^{3}$ (334 mg, $0.546 \mathrm{mmol}$ ) in $\operatorname{DMF}(3.3 \mathrm{~mL})$ was added $\mathrm{Pd}(\mathrm{MeCN}){ }_{2} \mathrm{Cl}_{2}$ at $0^{\circ} \mathrm{C}$. The resultant mixture was stirred at the same temperature for $12 \mathrm{~h}$. After the addition of $\mathrm{NaHCO}_{3}$ solution, the reaction mixture was extracted with EtOAc. The combined organic layers were washed with $\mathrm{H}_{2} \mathrm{O}$ and saturated $\mathrm{NaCl}$ solution, dried over $\mathrm{Na}_{2} \mathrm{SO}_{4}$, and concentrated under reduced pressure. 
The resultant residue was purified by silica gel column chromatography (hexane/EtOAc $=6 / 1$ to $4 / 1$ ) to afford the triene $(91 \mathrm{mg}, 96 \%)$ as a colorless oil. $[\alpha]_{\mathrm{D}}^{24}+7.1$ ( $\mathrm{C}$ $\left.0.21, \mathrm{CHCl}_{3}\right)$. IR $v_{\max }$ (neat) : 3507, 2955, 1737, 1252, 1112, $836 \mathrm{~cm}^{-1} .{ }^{1} \mathrm{H}-\mathrm{NMR}(600 \mathrm{MHz}$, $\left.\mathrm{CDCl}_{3}\right) \delta: 7.67-7.70(4 \mathrm{H}, \mathrm{m}), 7.36-7.45(6 \mathrm{H}, \mathrm{m}), 6.78(1 \mathrm{H}, \mathrm{dd}, \mathrm{J}=9.8,4.1 \mathrm{~Hz})$, $6.77(1 \mathrm{H}, \mathrm{dd}, J=15.0,11.2 \mathrm{~Hz}), 6.34(1 \mathrm{H}, t, J=11.3 \mathrm{~Hz}), 6.13(1 \mathrm{H}, t, J=11.3$ $\mathrm{Hz}), 6.05(1 \mathrm{H}, \mathrm{t}, J=11.3 \mathrm{~Hz}), 6.04(1 \mathrm{H}, \mathrm{d}, J=9.8 \mathrm{~Hz}), 5.96(1 \mathrm{H}, \mathrm{dd}, J=15.7$, $6.0 \mathrm{~Hz}), 5.92(1 \mathrm{H}, \mathrm{d}, J=15.8 \mathrm{~Hz}), 5.83(1 \mathrm{H}, \mathrm{dt}, J=15.0,4.6 \mathrm{~Hz}), 5.55(1 \mathrm{H}, \mathrm{dd}$, $J=11.3,7.8 \mathrm{~Hz}), 4.93(1 \mathrm{H}, \mathrm{td}, J=7.8,2.6 \mathrm{~Hz}), 4.81(1 \mathrm{H}, \mathrm{dd}, J=6.0,4.1 \mathrm{~Hz})$, $4.35(1 \mathrm{H}, t, J=4.1 \mathrm{~Hz}), 4.29(2 \mathrm{H}, \mathrm{d}, J=4.6 \mathrm{~Hz}), 3.69(1 \mathrm{H}, \mathrm{d}, J=10.8 \mathrm{~Hz}), 3.02$ $(1 \mathrm{H}, \mathrm{br}, \mathrm{OH}), 1.72(1 \mathrm{H}, \mathrm{dd}, J=13.9,7.8 \mathrm{~Hz}), 1.35(1 \mathrm{H}, \mathrm{ddd}, J=13.9,10.8,2.6$ $\mathrm{Hz}), 1.34(3 \mathrm{H}, \mathrm{s}), 1.08(9 \mathrm{H}, \mathrm{s}), 0.92(9 \mathrm{H}, \mathrm{t}, J=8.0 \mathrm{~Hz}), 0.90(9 \mathrm{H}, \mathrm{s}), 0.88(9 \mathrm{H}$, s), $0.57(6 \mathrm{H}, q, J=8.0 \mathrm{~Hz}), 0.11(3 \mathrm{H}, \mathrm{s}), 0.10(3 \mathrm{H}, \mathrm{s}), 0.07(3 \mathrm{H}, \mathrm{s}), 0.04(3 \mathrm{H}$, s). ${ }^{13} \mathrm{C}-\mathrm{NMR}\left(100 \mathrm{MHz}, \mathrm{CDCl}_{3}\right) \delta_{\mathrm{C}}: 163.0,145.7,140.1,135.6,135.5(4 \mathrm{C}), 134.2,133.6$, $133.5,130.0,129.7(2 \mathrm{C}), 127.7(4 \mathrm{C}), 124.5,123.4,123.1,122.2,121.8,81.3,77.5$, $74.9,67.1,64.6,64.2,39.0,26.8,25.8(3 \mathrm{C}), 25.6(3 \mathrm{C}), 22.7$ (3C), 19.2, 18.1,

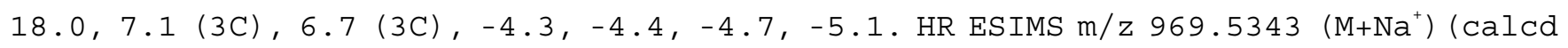
for 969.5342$)$.

\section{Diallyl}

$\left(1 E, 3 R, 4 R, 6 R, 7 Z, 9 Z, 11 E, 5^{\prime} S, 6^{\prime} S\right)-6$-tert-Butyldimethylsiloxy-1-(5' - tert-butyldime thylsiloxy-5',6'-dihydro-2' -oxo-2H-pyran-6'-yl)-13-tert-butyldiphenylsiloxy-3-m ethyl-3-triethylsiloxy-1,7,9,11-tridecatetraen-4-yl Phosphate (21)

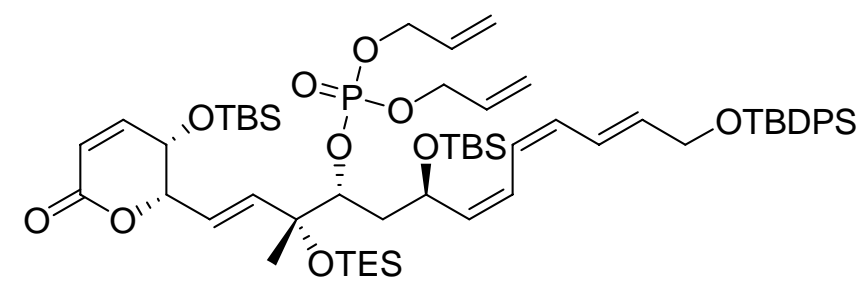

To a stirred solution of the triene $(91 \mathrm{mg}, 0.096 \mathrm{mmol}$ ) and tetrazole (34 mg, 0.49 mmol) in $\mathrm{CH}_{2} \mathrm{Cl}_{2}(2 \mathrm{~mL})$ was added $i-\mathrm{Pr}_{2} \mathrm{NP}(\mathrm{OAllyl})_{2}(132 \mu \mathrm{l}, 0.12 \mathrm{mmol})$ at room temperature. The resultant mixture was stirred at the same temperature for $15 \mathrm{~min}$, then $\mathrm{MeOH}$ ( 1 drop) was added. The resultant mixture was stirred at $0{ }^{\circ} \mathrm{C}$ for $5 \mathrm{~min}$, then TBHP (3.2M in $\mathrm{CH}_{2} \mathrm{Cl}_{2}, 900 \mu \mathrm{l}, 0.281 \mathrm{mmol}$ ) was added, and stirred for $1.5 \mathrm{~h}$ at the same temperature. Then additional TBHP (3.2M in $\mathrm{CH}_{2} \mathrm{Cl}_{2}, 300 \mu \mathrm{l}, 0.094 \mathrm{mmol}$ ) was added to the mixture, and the reaction mixture was stirred for $1 \mathrm{~h}$ at the same temperature. Additional TBHP (3.2M in $\mathrm{CH}_{2} \mathrm{Cl}_{2}, 300 \mu \mathrm{l}, 0.094 \mathrm{mmol}$ ) was added, and the solution was stirred for another $1 \mathrm{~h}$. After the addition of saturated $\mathrm{Na}_{2} \mathrm{~S}_{2} \mathrm{O}_{3}$ solution, the reaction mixture was extracted with EtOAc. The combined organic layers were washed with $\mathrm{H}_{2} \mathrm{O}$ and saturated $\mathrm{NaCl}$ solution, dried over $\mathrm{Na}_{2} \mathrm{SO}_{4}$, and concentrated under reduced pressure. The 
resultant residue was purified by silica gel column chromatography (hexane/EtOAC/Et ${ }_{3} \mathrm{~N}$

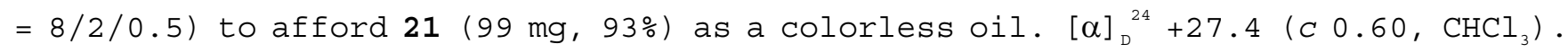
IR $v_{\max }$ (neat): 2956, 1735, 1252, 1112, $1020 \mathrm{~cm}^{-1} \cdot{ }^{1} \mathrm{H}-\mathrm{NMR}\left(600 \mathrm{MHz}, \mathrm{CDCl}_{3}\right) \delta: 7.66-$ $7.70(4 \mathrm{H}, \mathrm{m}), 7.41-7.45(2 \mathrm{H}, \mathrm{m}), 7.36-7.41(4 \mathrm{H}, \mathrm{m}), 6.77(1 \mathrm{H}, \mathrm{t}, J=15.0,11.4$ $\mathrm{Hz}), 6.70(1 \mathrm{H}, \mathrm{dd}, J=9.8,4.5 \mathrm{~Hz}), 6.30(1 \mathrm{H}, t, J=11.4 \mathrm{~Hz}), 6.23(1 \mathrm{H}, t, J=$ $11.4 \mathrm{~Hz}), 6.02(1 \mathrm{H}, \mathrm{d}, J=9.8 \mathrm{~Hz}), 6.02(1 \mathrm{H}, \mathrm{t}, J=11.4 \mathrm{~Hz}), 5.91-6.00(2 \mathrm{H}, \mathrm{m})$, $5.99(1 \mathrm{H}, \mathrm{dd}, J=15.6,7.0 \mathrm{~Hz}), 5.88(1 \mathrm{H}, \mathrm{d}, J=15.6 \mathrm{~Hz}), 5.83(1 \mathrm{H}, \mathrm{dt}, J=15.0$, $5.0 \mathrm{~Hz}), 5.41(1 \mathrm{H}, \mathrm{dd}, J=11.4,10.0 \mathrm{~Hz}), 5.39(1 \mathrm{H}, \mathrm{dd}, J=17.2,10.0 \mathrm{~Hz}), 5.39$ $(1 \mathrm{H}, \mathrm{dd}, J=17.2,10.0 \mathrm{~Hz}), 5.27(1 \mathrm{H}, t, J=10.0 \mathrm{~Hz}), 5.27(1 \mathrm{H}, t, J=10.0 \mathrm{~Hz})$, $4.97(1 \mathrm{H}, t, J=10.0 \mathrm{~Hz}), 4.78(1 \mathrm{H}, \mathrm{dd}, J=7.0,3.3 \mathrm{~Hz}), 4.53-4.60(4 \mathrm{H}, \mathrm{m}), 4.48$ $(1 \mathrm{H}, t, J=9.1 \mathrm{~Hz}), 4.30(1 \mathrm{H}, \mathrm{dd} J=4.5,3.3 \mathrm{~Hz}), 4.28(2 \mathrm{H}, \mathrm{d}, J=5.0 \mathrm{~Hz}), 2.00$ $-2.06(1 \mathrm{H}, \mathrm{m}), 1.46(3 \mathrm{H}, \mathrm{s}), 1.22-1.28(1 \mathrm{H}, \mathrm{m}), 1.07(9 \mathrm{H}, \mathrm{s}), 0.95(9 \mathrm{H}, t, J=$ $8.0 \mathrm{~Hz}), 0.88(18 \mathrm{H}, \mathrm{s}), 0.63(6 \mathrm{H}, \mathrm{q}, J=8.0 \mathrm{~Hz}), 0.12(3 \mathrm{H}, \mathrm{s}), 0.08(3 \mathrm{H}, \mathrm{s}), 0.07$ $(3 \mathrm{H}, \mathrm{s}), 0.04(3 \mathrm{H}, \mathrm{s}) \cdot{ }^{13} \mathrm{C}-\mathrm{NMR}\left(100 \mathrm{MHz}, \mathrm{CDCl}_{3}\right) \delta_{\mathrm{C}}: 163.1,145.8,139.0,136.4,135.5$ $(4 \mathrm{C}), 134.0,133.6(2 \mathrm{C}), 132.6\left(\mathrm{~d}, J_{\mathrm{C}-\mathrm{P}}=7.5 \mathrm{~Hz}\right), 132.6\left(\mathrm{~d}, J_{\mathrm{C}-\mathrm{P}}=7.4 \mathrm{~Hz}\right), 129.8$, $129.7(2 \mathrm{C}), 127.7(4 \mathrm{C}), 124.6,123.7,123.5,122.1,121.8,118.3,118.1,82.5(d$, $\left.J_{\mathrm{C}-\mathrm{P}}=7.2 \mathrm{~Hz}\right), 81.4,76.2\left(\mathrm{~d}, J_{\mathrm{C}-\mathrm{P}}=5.2 \mathrm{~Hz}\right), 68.2\left(\mathrm{~d}, J_{\mathrm{C}-\mathrm{P}}=5.5 \mathrm{~Hz}\right), 68.1\left(\mathrm{~d}, J_{\mathrm{C}-\mathrm{P}}=\right.$ $5.4 \mathrm{~Hz}), 64.9,64.4,64.2,40.2\left(\mathrm{~d}, J_{\mathrm{C}-\mathrm{P}}=3.4 \mathrm{~Hz}\right), 26.8(3 \mathrm{C}), 26.0(3 \mathrm{C}), 25.7$ (3C), $25.6,19.2,18.1,18.0,7.2(3 \mathrm{C}), 6.8(3 \mathrm{C}),-4.1,-4.3,-4.4,-4.6 .{ }^{31} \mathrm{P}-\mathrm{NMR}(162 \mathrm{MHz}$, $\left.\mathrm{CDCl}_{3}\right) \delta_{\mathrm{P}}:-1.2$. HR ESIMS m/z $1129.5636\left(\mathrm{M}+\mathrm{Na}^{+}\right)$(calcd for 1129.5632).

$\left(1 E, 3 R, 4 R, 6 R, 7 Z, 9 Z, 11 E, 5^{\prime} S, 6^{\prime} S\right)-1-\left(5^{\prime}, 6^{\prime}\right.$-dihydro-5'-hydroxy-2'-oxo-2H-pyran-6' yl) - 3,6,13-trihydroxy-3-methyl-1,7,9,11-tridecatetraen-4-yl Sodium Phosphate (1a)<smiles>C[C@](O)(/C=C/[C@H]1OC(=O)C=C[C@@H]1O)[C@@H](C[C@H](O)/C=C\C=C/C=C/CO)OP(=O)(O)O[Na]</smiles>

To a stirred solution of $21(10.3 \mathrm{mg}, 9.3 \mu \mathrm{mol})$ in degassed THF $(370 \mu \mathrm{l})$ were added $\mathrm{Et}_{3} \mathrm{~N}(9.7 \mu \mathrm{l}, 0.07 \mathrm{mmol}), \mathrm{HCO}_{2} \mathrm{H}(10.5 \mu \mathrm{l}, 0.28 \mathrm{mmol})$ and $\mathrm{Pd}\left(\mathrm{PPh}_{3}\right)_{4}(1.1 \mathrm{mg}, 1 \mu \mathrm{mol})$ in this order at room temperature. The resultant mixture was stirred at $50{ }^{\circ} \mathrm{C}$ for $1 \mathrm{~h}$. Then the mixture was concentrated under reduced pressure to give the crude phosphate, which was used directly to the next step.

The reaction was performed in a Teflon vessel. To a stirred solution of the crude phosphate above in $\mathrm{MeCN}(370 \mu \mathrm{l})$ was added $\mathrm{Et}_{3} \mathrm{~N} \cdot 3 \mathrm{HF}(30 \mu \mathrm{l}, 0.19 \mathrm{mmol})$, followed by $\mathrm{Et}_{3} \mathrm{~N}(13 \mu \mathrm{l}, 0.093 \mathrm{mmol})$ at $60{ }^{\circ} \mathrm{C}$ for $2 \mathrm{~h}$. The reaction was quenched by addition of $\mathrm{NaHCO}_{3}\left(0.5 \mathrm{M}\right.$ in $\left.\mathrm{H}_{2} \mathrm{O}, 2.2 \mathrm{~mL}, 1.1 \mathrm{mmol}\right)$ and washed with EtOAC. The aqueous layer was concentrated under reduced pressure. The resultant residue was purified by $\mathrm{C}_{18}$-reverse 
phase silica gel column chromatography ( $\mathrm{H}_{2} \mathrm{O}$ only to $\mathrm{H}_{2} \mathrm{O} / \mathrm{MeCN}=9 / 1$ ) to afford PD 113,271 (1a) $(4.0 \mathrm{mg}, 92 \%)$ as white crystals. $[\alpha]_{\mathrm{D}}{ }^{24}+39.6\left(\mathrm{C} 0.073, \mathrm{H}_{2} \mathrm{O}\right)$. IR $v_{\max }(\mathrm{KBr}): 3443$, $1701,1559,1074,970,829 \mathrm{~cm}^{-1} \cdot{ }^{1} \mathrm{H}-\mathrm{NMR}\left(600 \mathrm{MHz}, \mathrm{D}_{2} \mathrm{O}\right) \delta: 7.14(1 \mathrm{H}, \mathrm{dd}, J=9.8,5.9$ $\mathrm{Hz}), 6.77(1 \mathrm{H}, \mathrm{dd}, J=15.0,11.5), 6.56(1 \mathrm{H}, \mathrm{br} t, J=11.5 \mathrm{~Hz}), 6.37(1 \mathrm{H}, t, J$ $=11.5 \mathrm{~Hz}), 6.16(1 \mathrm{H}, \mathrm{d}, J=11.5 \mathrm{~Hz}), 6.16(1 \mathrm{H}, t, J=9.8 \mathrm{~Hz}), 6.00(1 \mathrm{H}, \mathrm{d}, J=$ $15.9 \mathrm{~Hz}), 5.93(1 \mathrm{H}, \mathrm{dt}, J=15.0,5.6 \mathrm{~Hz}), 5.93(1 \mathrm{H}, \mathrm{dd}, J=15.9,6.0 \mathrm{~Hz}), 5.53$ $(1 \mathrm{H}$, br $t, J=9.8 \mathrm{~Hz}), 5.08(1 \mathrm{H}, \mathrm{dd}, J=6.0,2.8 \mathrm{~Hz}), 4.93(1 \mathrm{H}, \mathrm{br}$ td, $J=9.6$, $2.1 \mathrm{~Hz}), 4.34(1 \mathrm{H}, \mathrm{dd}, J=5.9,2.8 \mathrm{~Hz}), 4.16(2 \mathrm{H}, \mathrm{d}, J=5.6 \mathrm{~Hz}), 4.14(1 \mathrm{H}, \mathrm{br}$ td, $J=10.6,2.1 \mathrm{~Hz}), 1.65(1 \mathrm{H}$, br $t, J=12.3 \mathrm{~Hz}), 1.56(1 \mathrm{H}, \mathrm{ddd}, J=14.2,11.2,2.8$ $\mathrm{Hz}), 1.29(3 \mathrm{H}, \mathrm{s}) \cdot{ }^{13} \mathrm{C}-\mathrm{NMR}\left(150 \mathrm{MHz}, \mathrm{D}_{2} \mathrm{O}\right) \delta_{\mathrm{C}}: 169.6,149.0,142.0,136.6,136.3,133.1$, $129.0,127.1,126.8,126.1,124.4,84.3,79.0\left(\mathrm{~d}, J_{\mathrm{C}-\mathrm{P}}=5.3 \mathrm{~Hz}\right) 78.4\left(\mathrm{~d}, J_{\mathrm{C}-\mathrm{P}}=1.7\right.$ $\mathrm{Hz}), 66.4,64.9,64.5,41.4\left(\mathrm{~d}, J_{\mathrm{C}-\mathrm{P}}=4.0 \mathrm{~Hz}\right), 23.4 .{ }^{31} \mathrm{P}-\mathrm{NMR}\left(162 \mathrm{MHz}, \mathrm{D}_{2} \mathrm{O}\right) \delta_{\mathrm{P}}: 6.0$. HR ESIMS m/z $491.1064\left(\mathrm{M}+\mathrm{Na}^{+}\right)$(calcd for 491.1053).

$\left(1 E, 3 R, 4 R, 6 R, 7 Z, 9 Z, 11 E, 5^{\prime} S, 6^{\prime} S\right)-6,13$-diacetoxy-1-(5'-acetoxy-5' , 6'-dihydro-2' -o xo-2H-pyran-6'-yl) -3,4-dihydroxy-3-methyl-1, 7,9,11-tridecatetraen-3,4-Cyclophos phate, Sodium Salt $(22 a)$<smiles>CC(=O)OC/C=C/C=C\C=C/[C@H](C[C@H]1OP(=O)(O[NH3+])O[C@@]1(C)/C=C/[C@@H]1OC(=O)C=C[C@@H]1OC(C)=O)OC(C)=O</smiles>

To a stirred solution of $1 \mathrm{a}(1.1 \mathrm{mg}, 3.3 \mu \mathrm{mol})$ in Py $(100 \mu \mathrm{l})$ was added $\mathrm{Ac}_{2} \mathrm{O}(200$ $\mu l)$ at $0{ }^{\circ} \mathrm{C}$. The resultant mixture was stirred at room temperture for $10 \mathrm{~h}$, then removal of the solvents gave the corresponding phosphate, which was dissolved in saturated $\mathrm{NaHCO}_{3}$ solution and chromatographed over $2 \mathrm{~mL}$ of HP-20 resin. Elution with methanol-water $(1: 1)$ and concentration of the eluate under reduced pressure yielded $\mathrm{PD} 116,251$ (22a) (1.1 mg, 81\%) as white crystals.

Synthetic PD 116,251: A white solid. $[\alpha]_{\mathrm{D}}{ }^{24}+28\left(\mathrm{C} 0.02, \mathrm{H}_{2} \mathrm{O}\right) \cdot{ }^{1} \mathrm{H}-\mathrm{NMR}\left(600 \mathrm{MHz}, \mathrm{D}_{2} \mathrm{O}\right)$ $\delta: 7.11(1 \mathrm{H}, \mathrm{dd}, J=9.7,5.8 \mathrm{~Hz}), 6.81(1 \mathrm{H}, \mathrm{dd}, J=15.0,11.5 \mathrm{~Hz}), 6.65(1 \mathrm{H}, t$, $J=11.5 \mathrm{~Hz}), 6.42(1 \mathrm{H}, t, J=11.5 \mathrm{~Hz}), 6.28(1 \mathrm{H}, \mathrm{d}, J=9.7 \mathrm{~Hz}), 6.20(1 \mathrm{H}, t, J$ $=11.5 \mathrm{~Hz}), 6.05(1 \mathrm{H}, \mathrm{d}, J=15.8,1.1 \mathrm{~Hz}), 5.98(1 \mathrm{H}, \mathrm{dd}, J=15.8,5.2 \mathrm{~Hz}), 5.91$ $(1 \mathrm{H}, \mathrm{dt}, J=15.0,6.4 \mathrm{~Hz}), 5.75(1 \mathrm{H}$, br $\mathrm{dt}, J=7.8,6.5 \mathrm{~Hz}), 5.50(1 \mathrm{H}, \mathrm{br} t, J$ $=10.0 \mathrm{~Hz}), 5.45(1 \mathrm{H}, \mathrm{dd}, J=5.8,2.8 \mathrm{~Hz}), 5.30(1 \mathrm{H}, \mathrm{ddd}, J=5.2,2.8,1.1 \mathrm{~Hz})$, $4.65(2 \mathrm{H}, \mathrm{d}, J=6.4 \mathrm{~Hz}), 4.24(1 \mathrm{H}, \mathrm{br} t, J=6.5 \mathrm{~Hz}), 2.08(3 \mathrm{H}, \mathrm{s}), 2.08(3 \mathrm{H}, \mathrm{s})$, $2.06(3 \mathrm{H}, \mathrm{s}), 1.95(2 \mathrm{H}, \mathrm{t}, J=6.5 \mathrm{~Hz}), 1.39(3 \mathrm{H}, \mathrm{s}) \cdot{ }^{31} \mathrm{P}-\mathrm{NMR}\left(162 \mathrm{MHz}, \mathrm{D}_{2} \mathrm{O}\right) \delta_{\mathrm{P}}: 14.5$. HR ESIMS m/z 599.1252 (M+Na ${ }^{+}$(calcd for 599.1264). 
Authentic sample of PD 116,251: A white solid. $[\alpha]_{\mathrm{D}}^{23}+29$ ( C $\left.0.04, \mathrm{H}_{2} \mathrm{O}\right){ }^{1} \mathrm{H}-\mathrm{NMR}$ (600 $\left.\mathrm{MHz}, \mathrm{D}_{2} \mathrm{O}\right) \delta: 7.11(1 \mathrm{H}, \mathrm{dd}, J=9.7,5.8 \mathrm{~Hz}), 6.81(1 \mathrm{H}, \mathrm{dd}, J=15.0,11.5 \mathrm{~Hz}), 6.65$ $(1 \mathrm{H}, t, J=11.5 \mathrm{~Hz}), 6.42(1 \mathrm{H}, t, J=11.5 \mathrm{~Hz}), 6.28(1 \mathrm{H}, d, J=9.7 \mathrm{~Hz}), 6.20(1 \mathrm{H}$, $t, J=11.5 \mathrm{~Hz}), 6.05(1 \mathrm{H}, \mathrm{d}, J=15.8,1.1 \mathrm{~Hz}), 5.98(1 \mathrm{H}, \mathrm{dd}, J=15.8,5.2 \mathrm{~Hz})$, $5.91(1 \mathrm{H}, \mathrm{dt}, J=15.0,6.4 \mathrm{~Hz}), 5.75(1 \mathrm{H}, \mathrm{br} d t, J=7.8,6.5 \mathrm{~Hz}), 5.50(1 \mathrm{H}, \mathrm{br}$ $t, J=10.0 \mathrm{~Hz}), 5.45(1 \mathrm{H}, \mathrm{dd}, J=5.8,2.8 \mathrm{~Hz}), 5.30(1 \mathrm{H}, \mathrm{ddd}, J=5.2,2.8,1.1$ $\mathrm{Hz}), 4.65(2 \mathrm{H}, \mathrm{d}, J=6.4 \mathrm{~Hz}), 4.24(1 \mathrm{H}, \mathrm{br} t, J=6.5 \mathrm{~Hz}), 2.08(3 \mathrm{H}, \mathrm{s}), 2.08(3 \mathrm{H}$, s), $2.06(3 \mathrm{H}, \mathrm{s}), 1.95(2 \mathrm{H}, \mathrm{t}, J=6.5 \mathrm{~Hz}), 1.39(3 \mathrm{H}, \mathrm{s})$. 
Reference

(1) Somfai, P.; Olsson, R. Tetrahedron 1993, 34, 6645.

(2) (a) Kiliani, H.; Kleeman, S. Ber. 1884, 17, 1296. (b) Bock, K. ; Lundt, I. ; Pedersen, C. Acta Chem. Scand. Ser. B 1981, B35, 155. (c) Nicolaou, K. C.; Fylaktakidou, K. C.; Monenschein, H.; Li, Y.; Weyershausen, B.; Mitchell, H. J.; Wei, H. ; Guntupalli, P.; Hepworth, D.; Sugita, K. J. Am. Chem. Soc. 2003, 125, 15433.

(3) (a) Mapp, A. K. ; Heathcock, C. H. J. Org. Chem. 1999, 64, 23. (b) Dabdoub, M. J.; Dabdoub, V. B.; Baroni, A. C. M. J. Am. Chem. Soc. 2001, 123, 9694. 
${ }^{1} \mathrm{H},{ }^{13} \mathrm{C}$ and ${ }^{31} \mathrm{P}$ NMR SPECTRAL DATA 


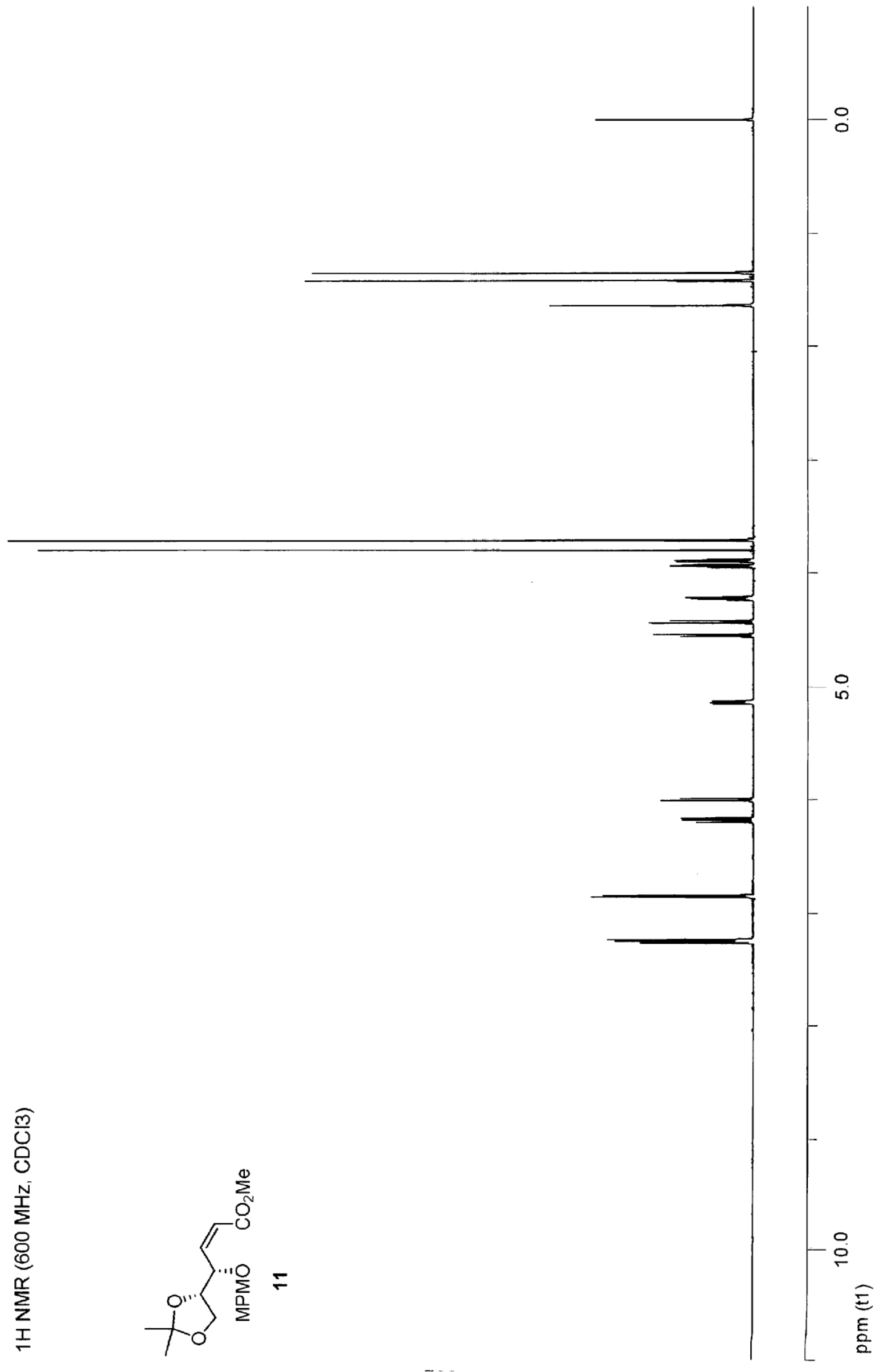




$$
\exists
$$




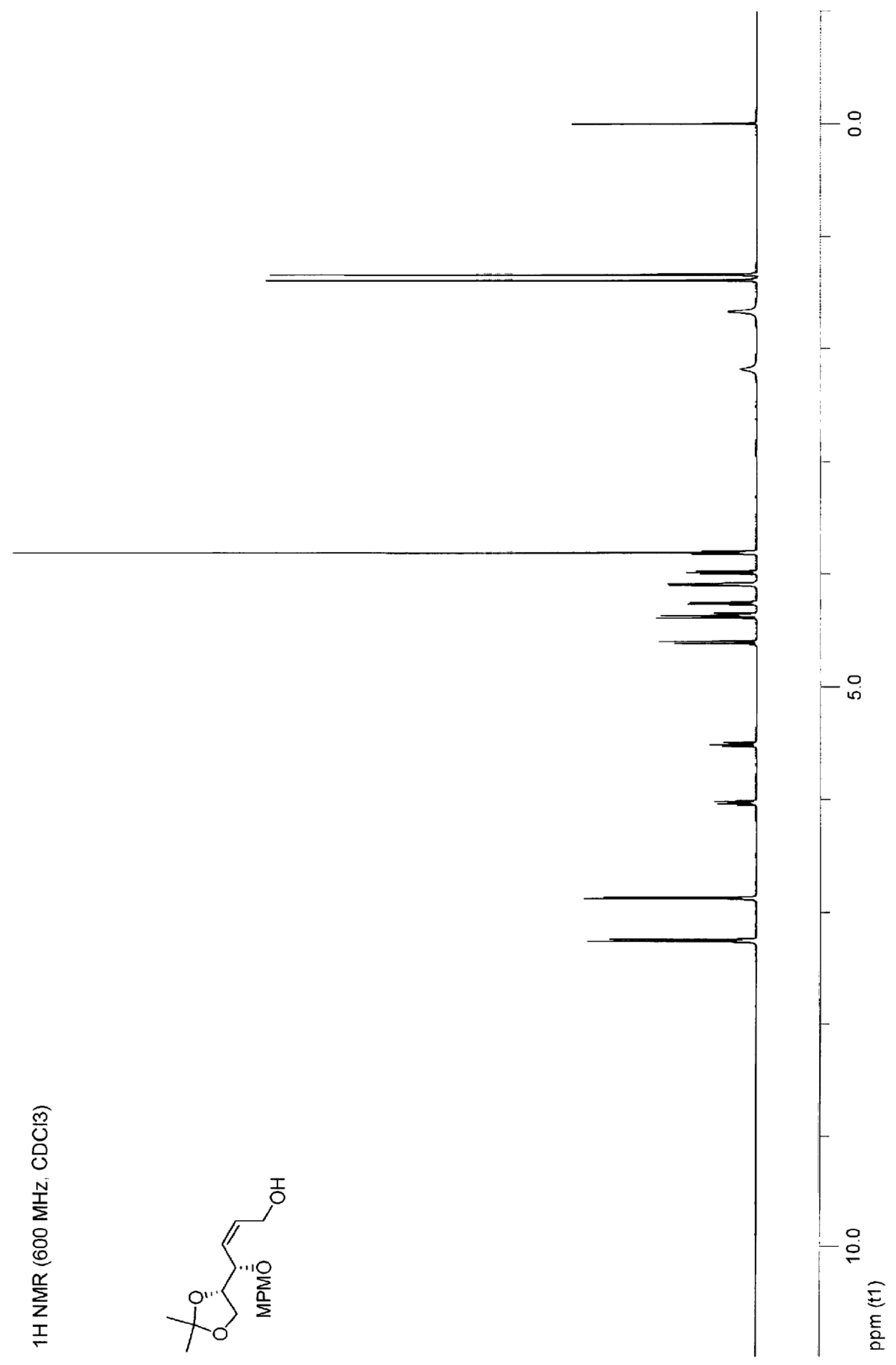




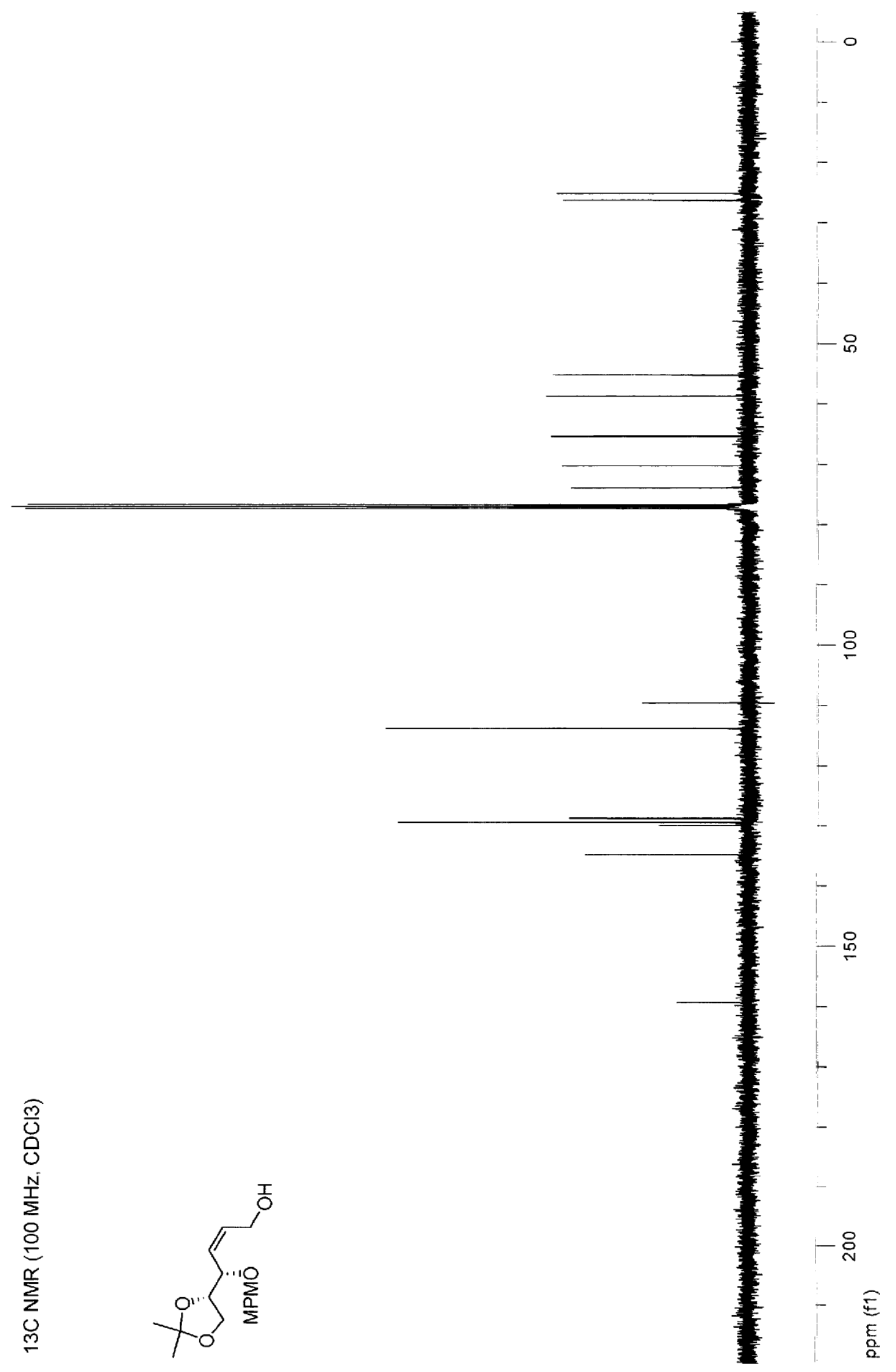




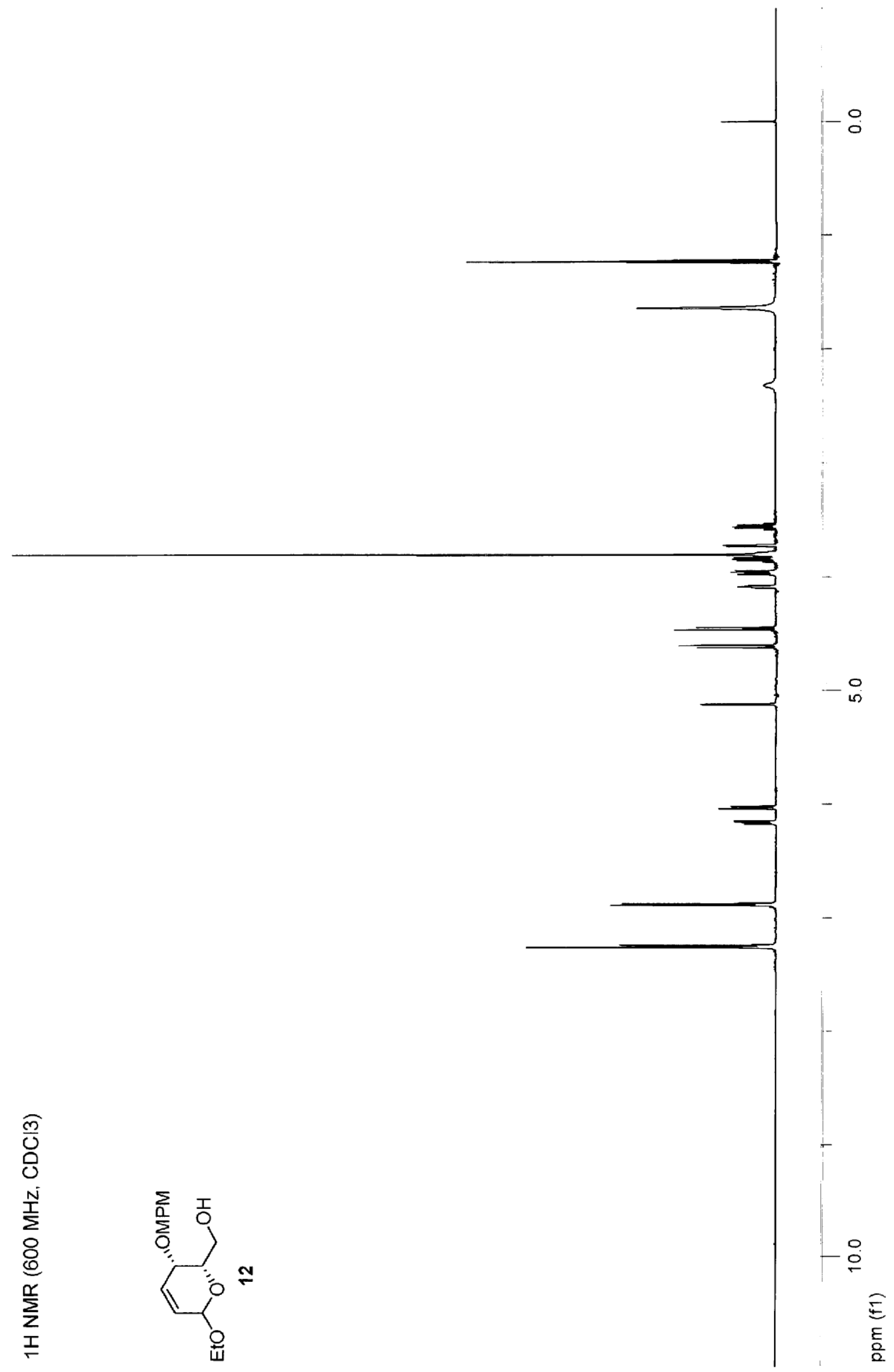




$$
7
$$




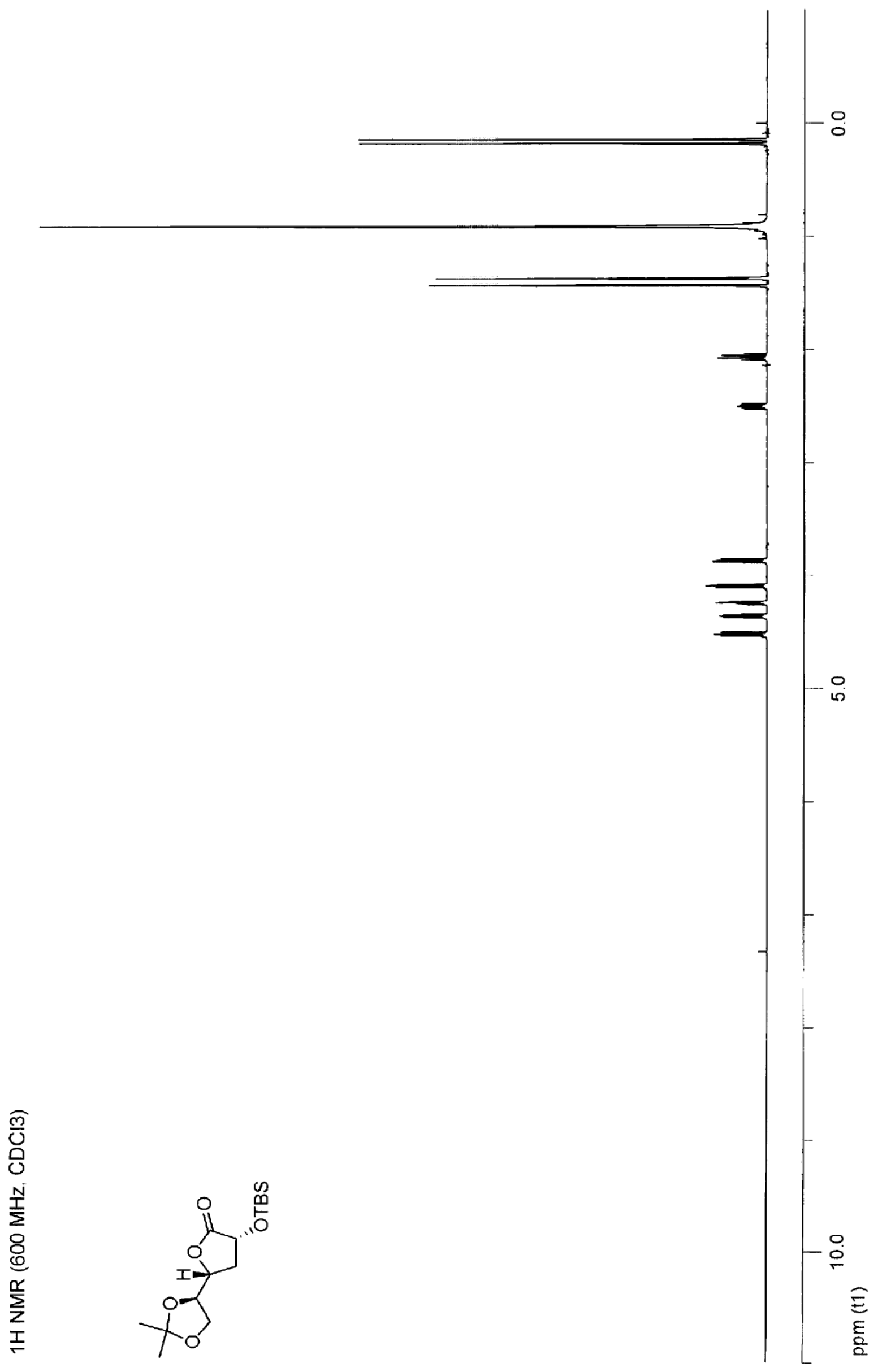




$$
4
$$




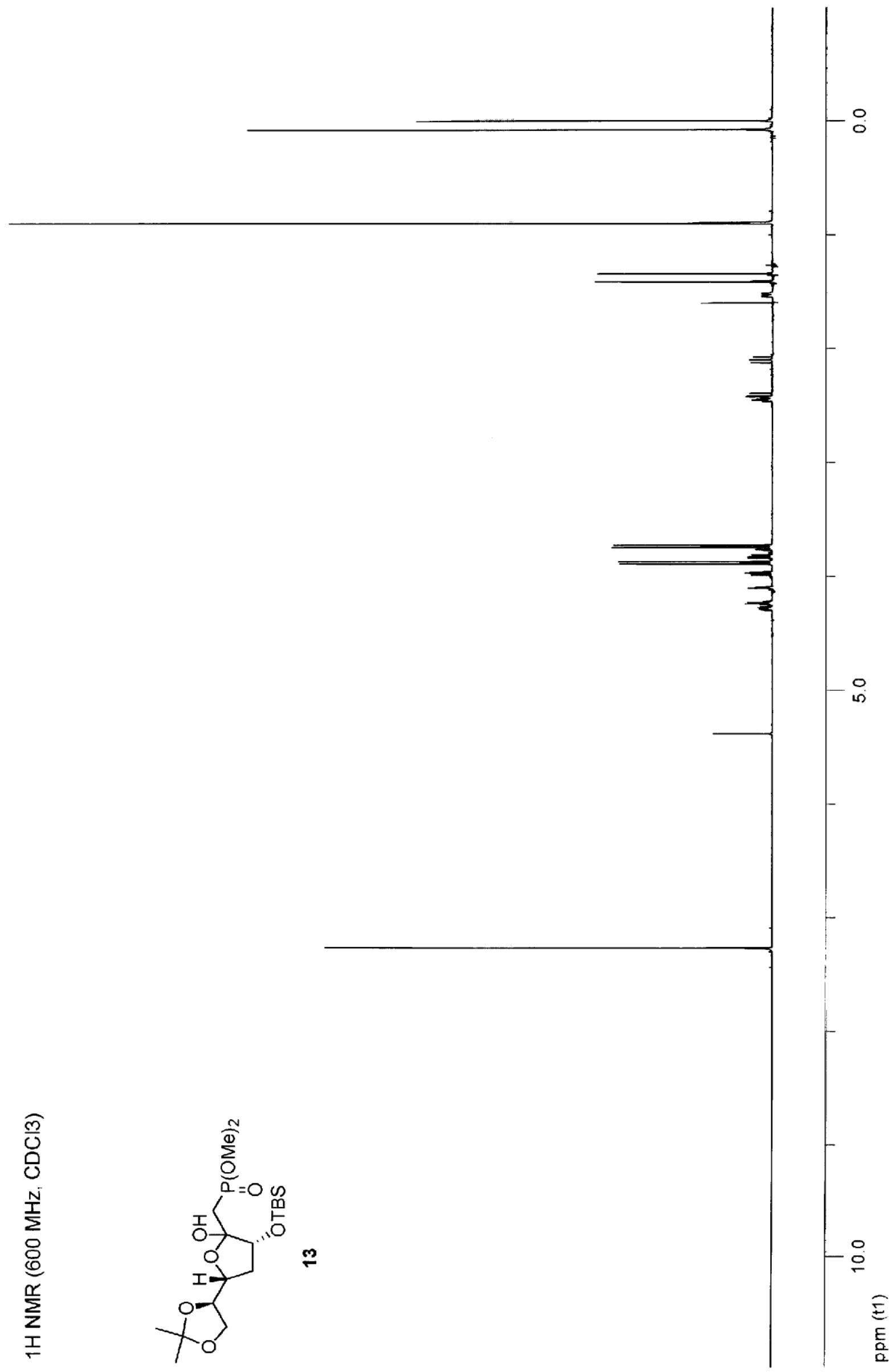




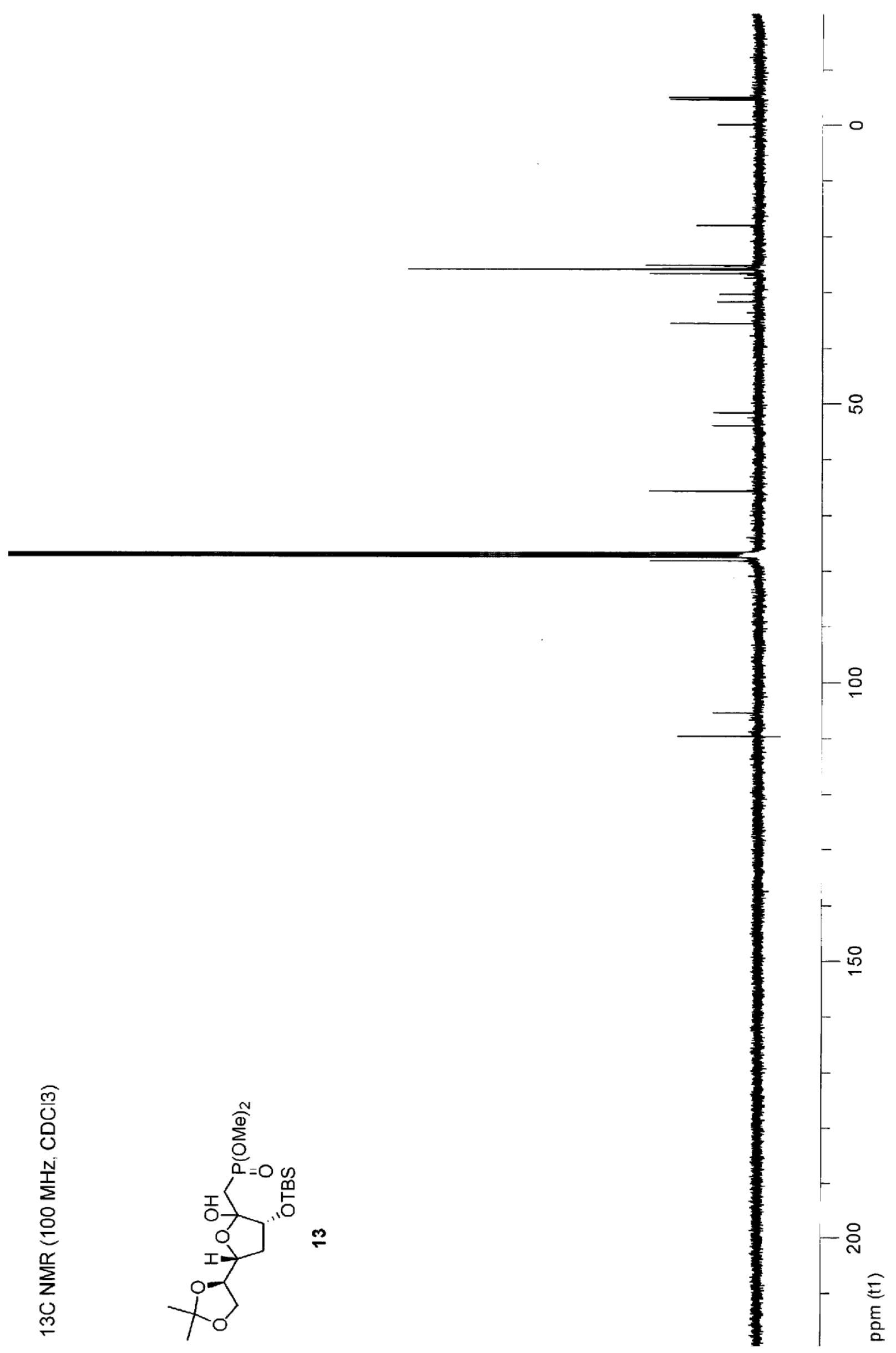




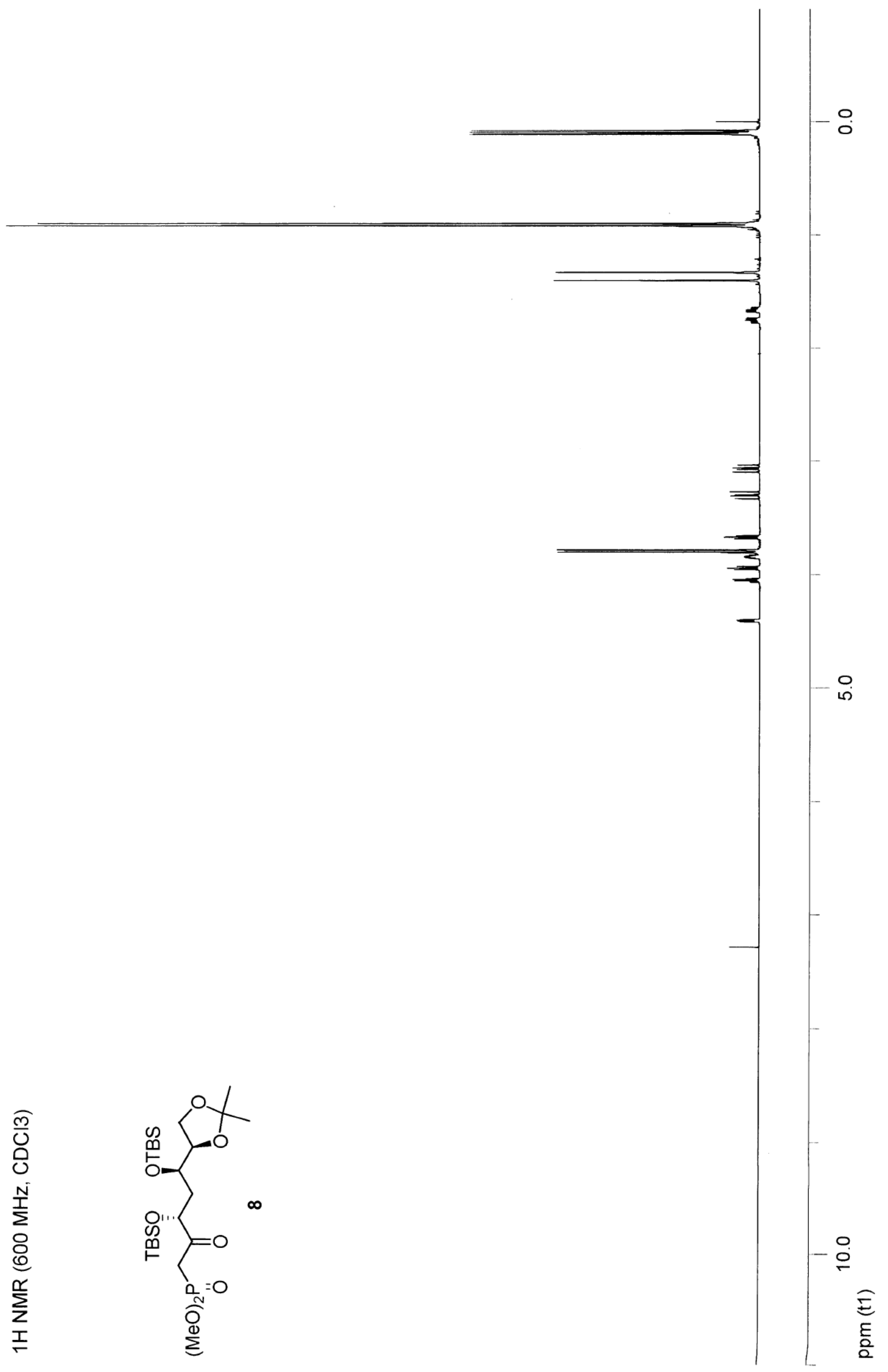




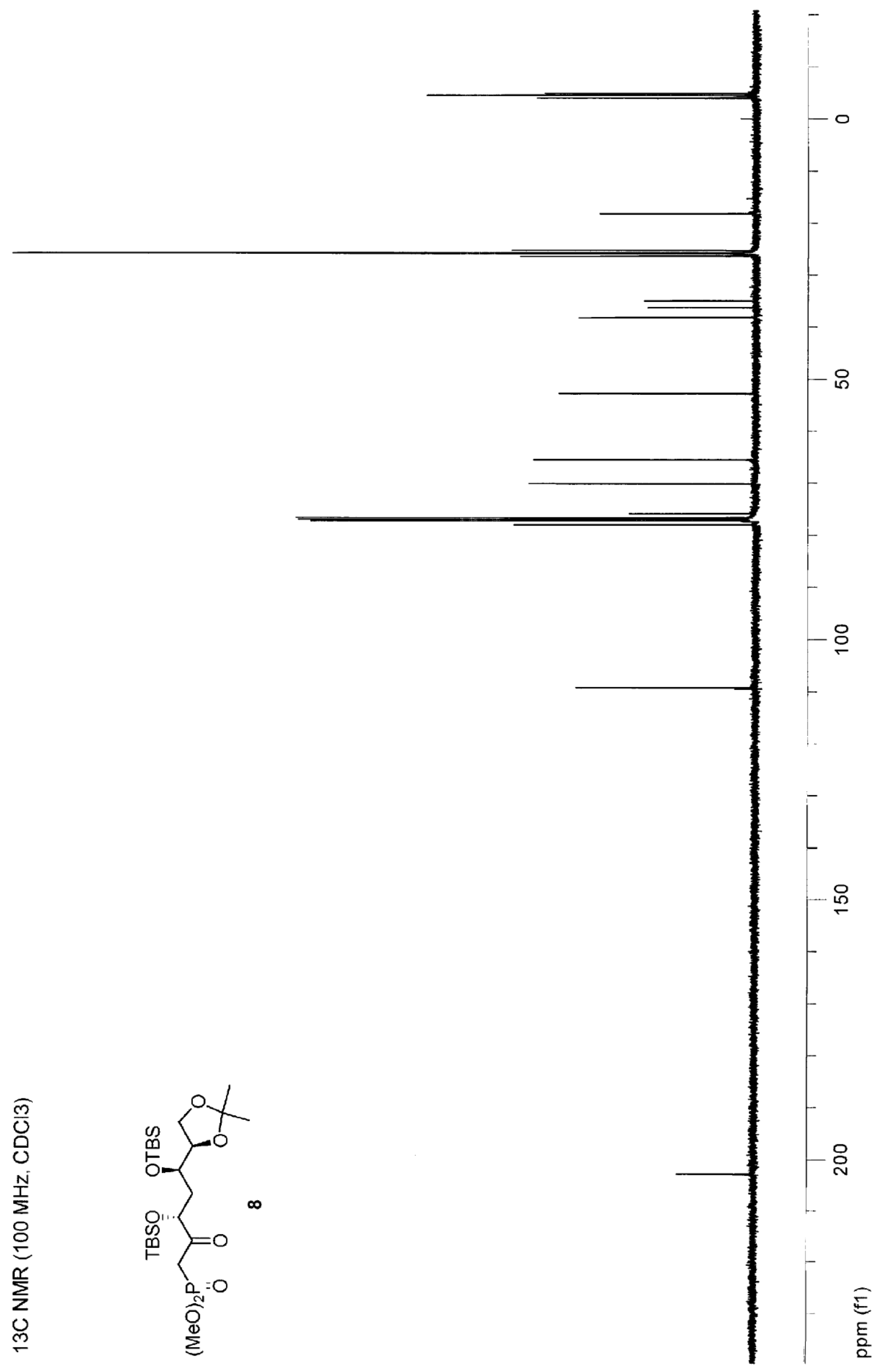




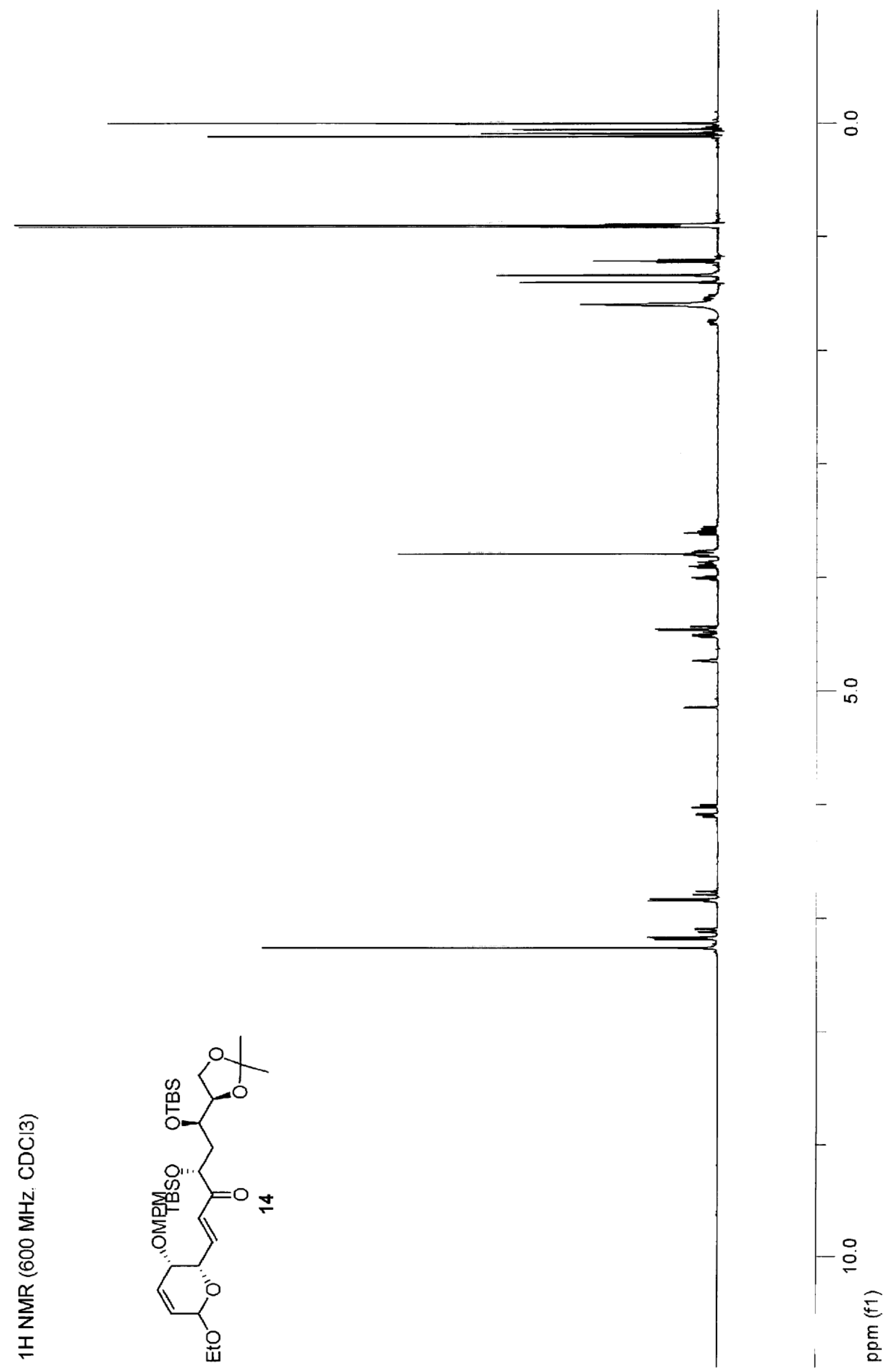




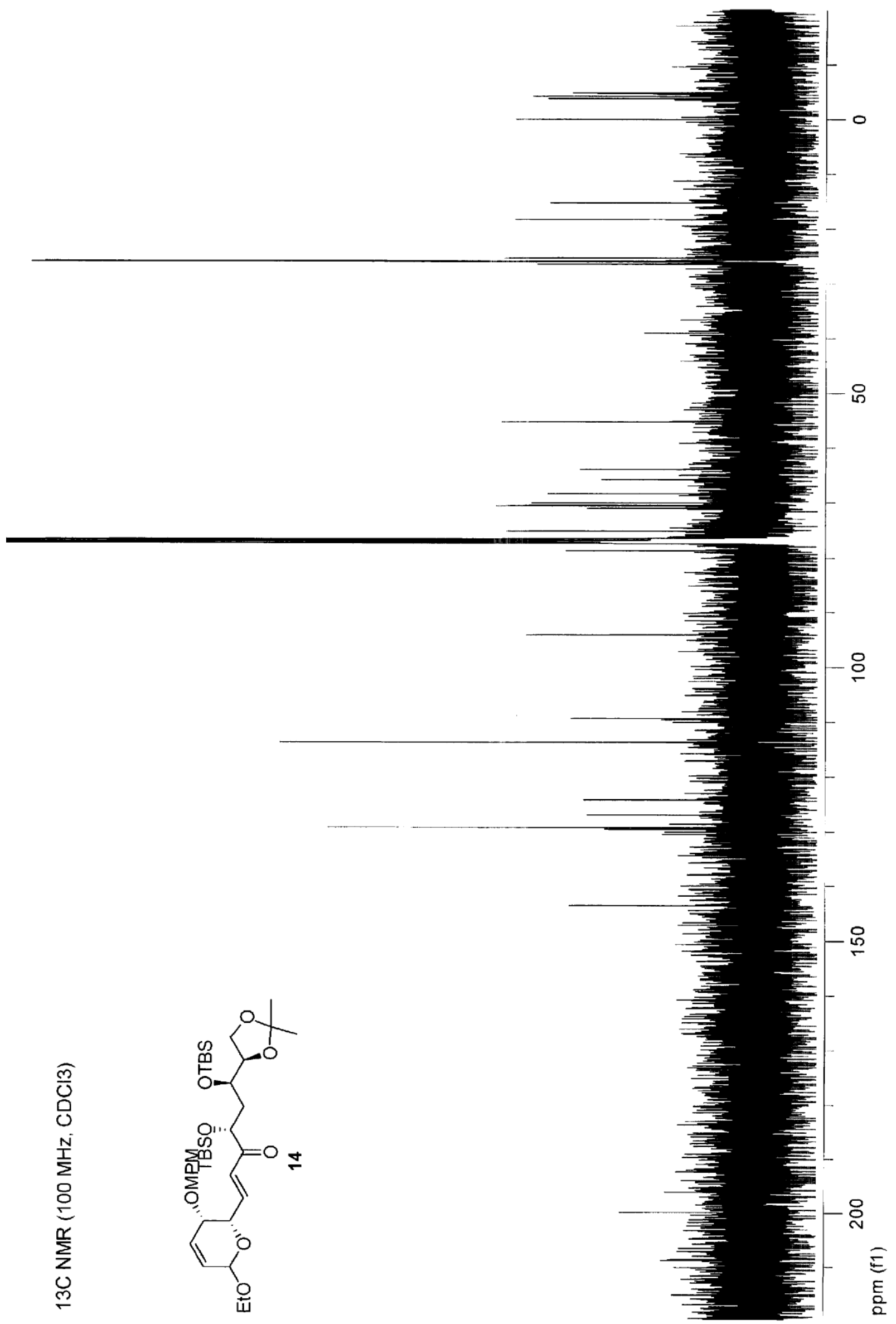




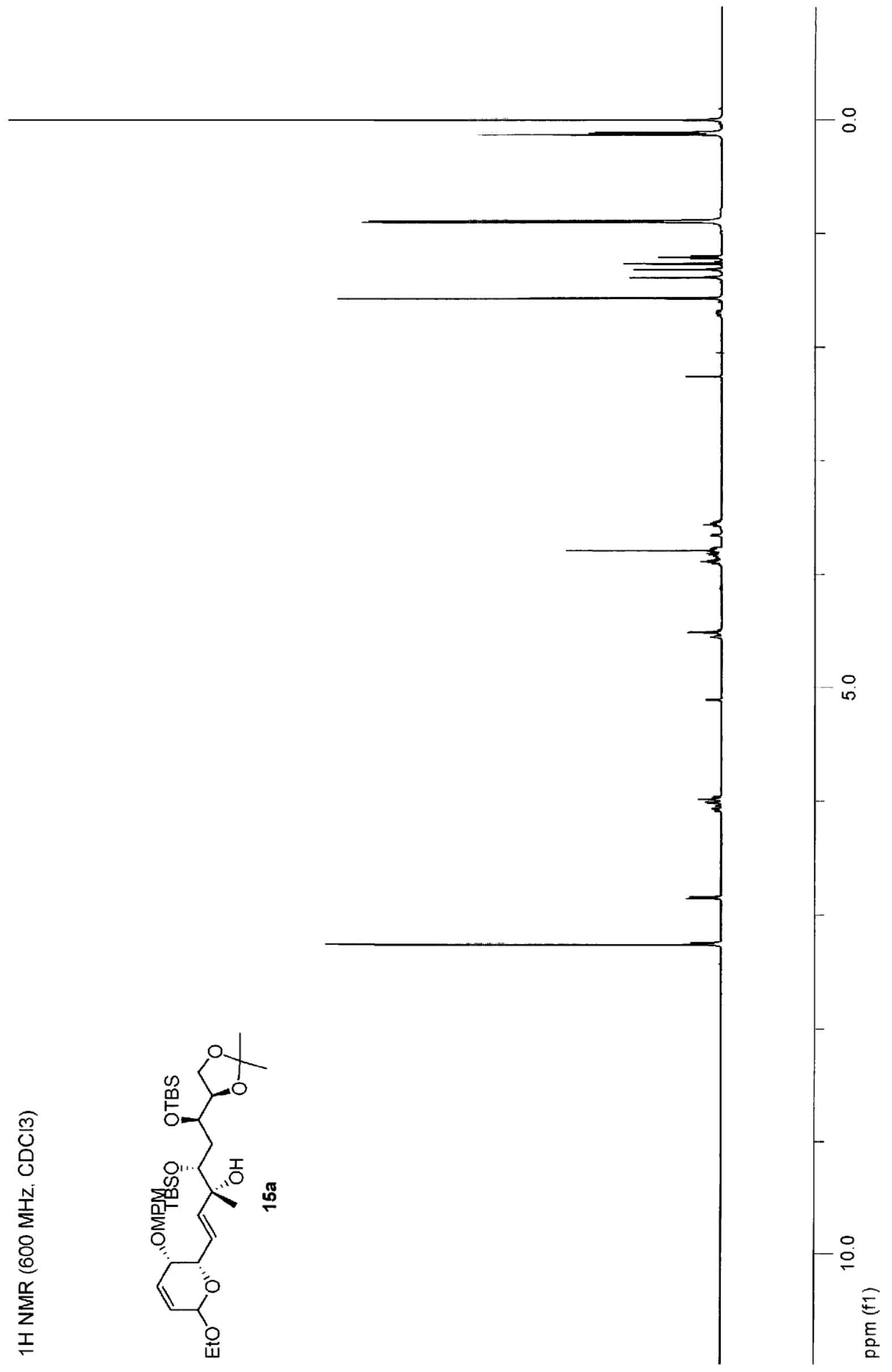




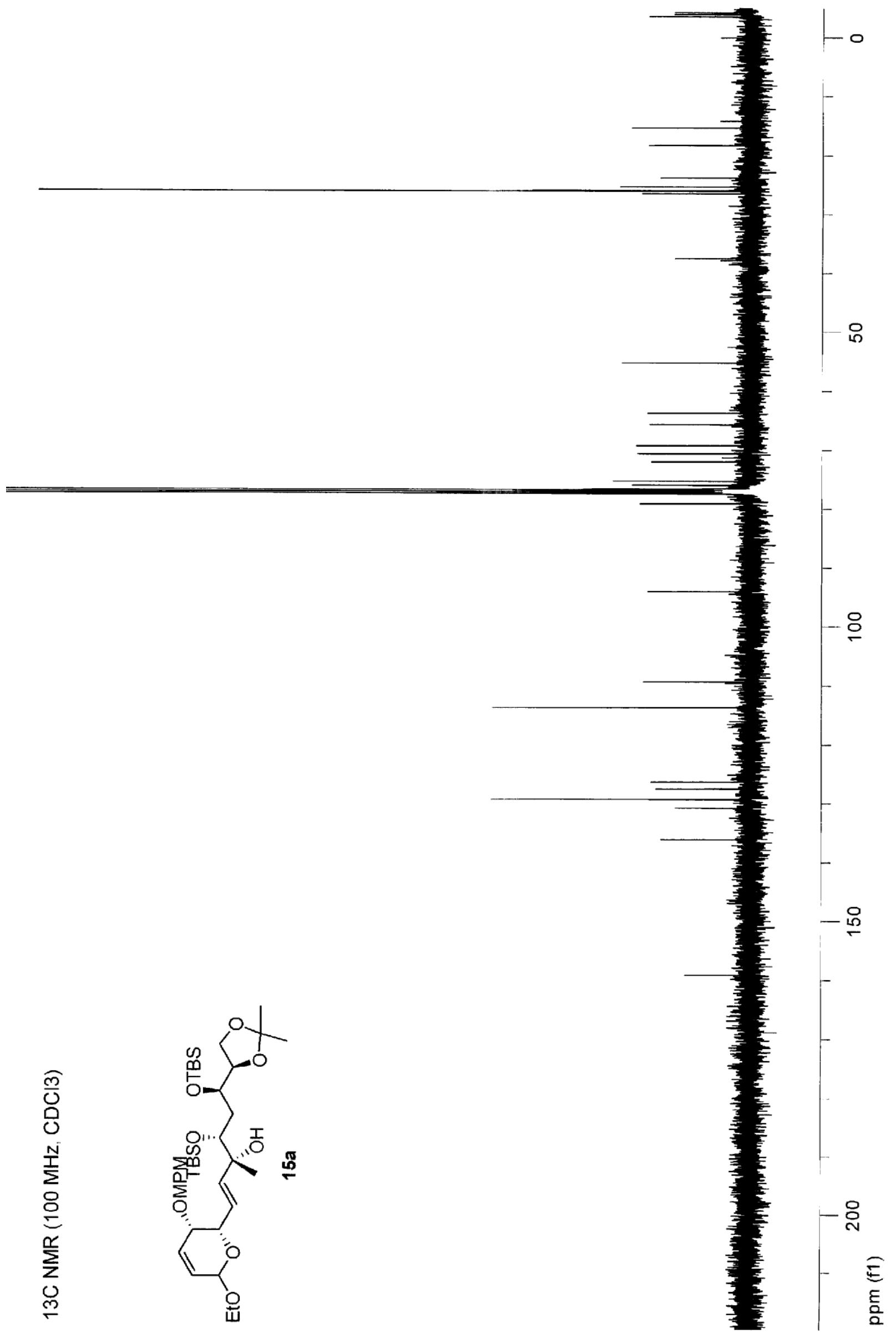




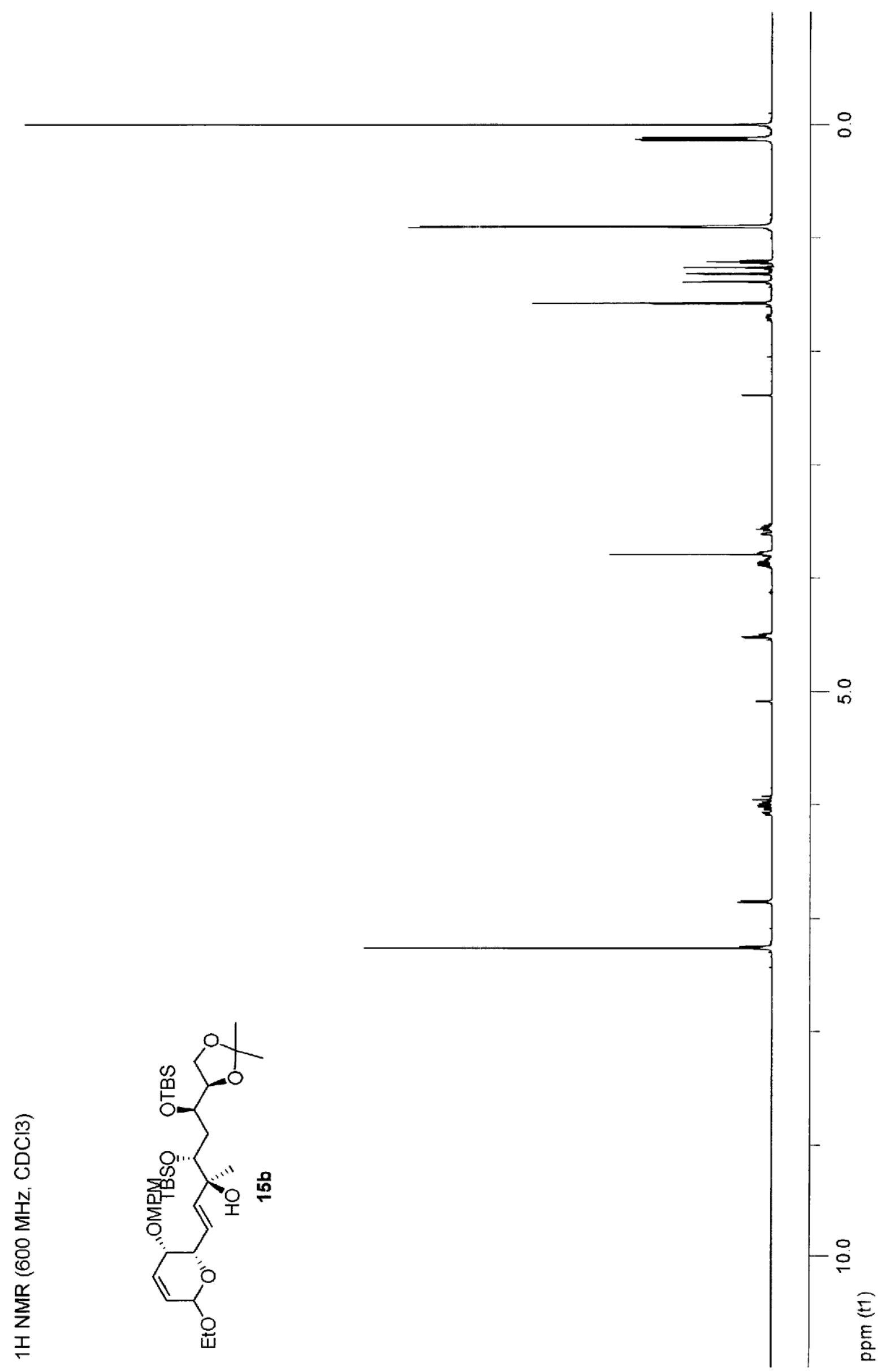




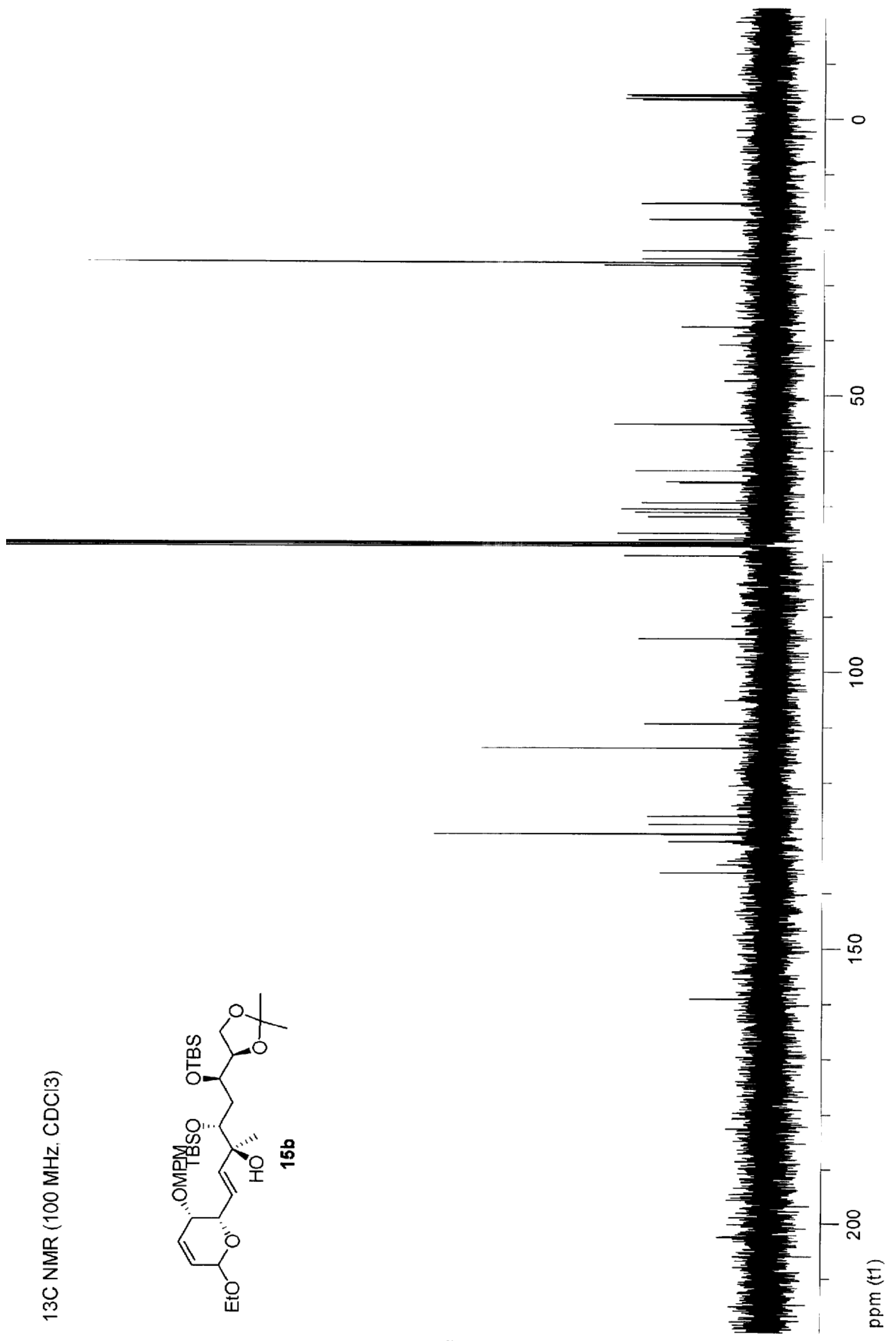




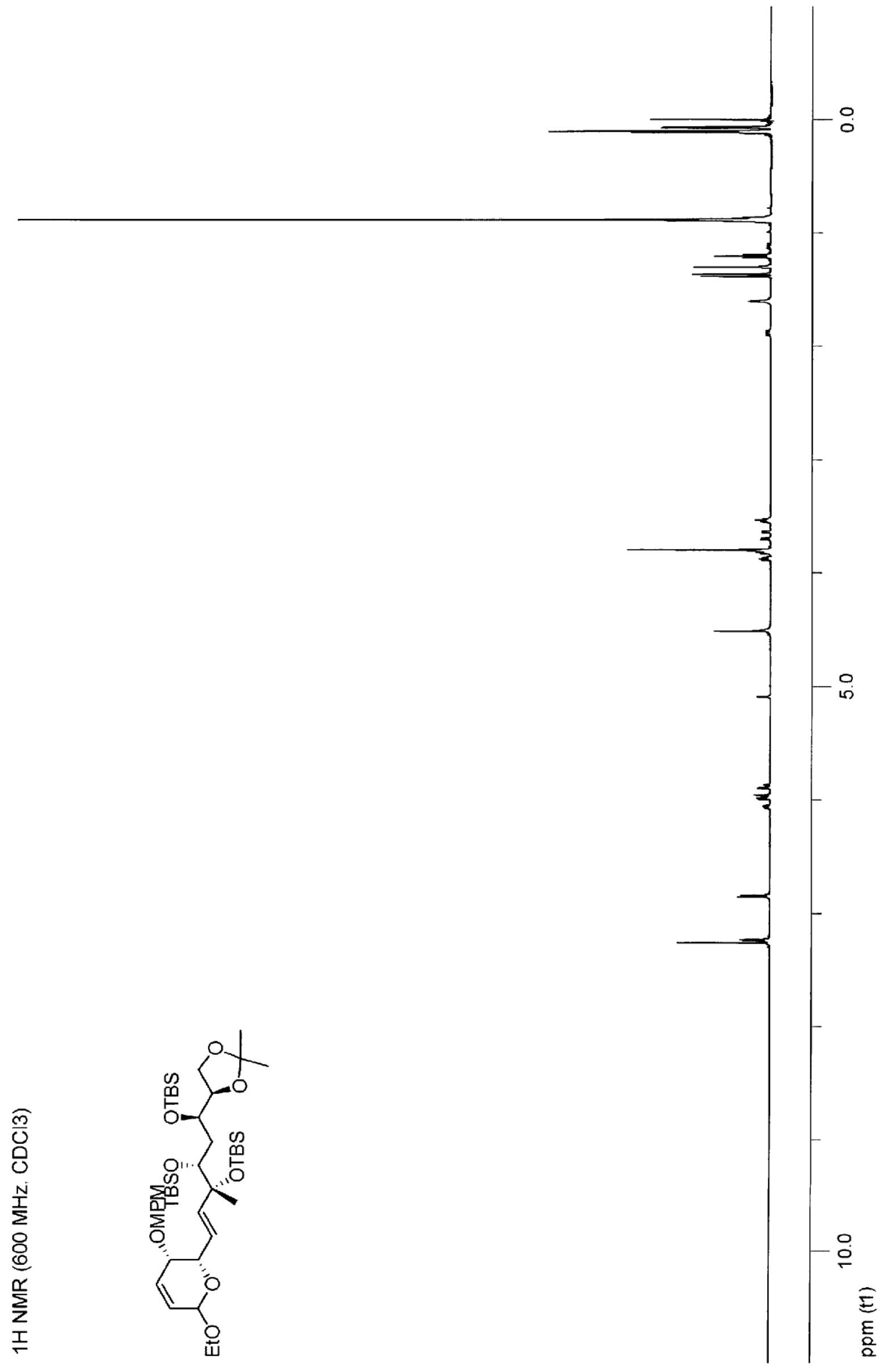




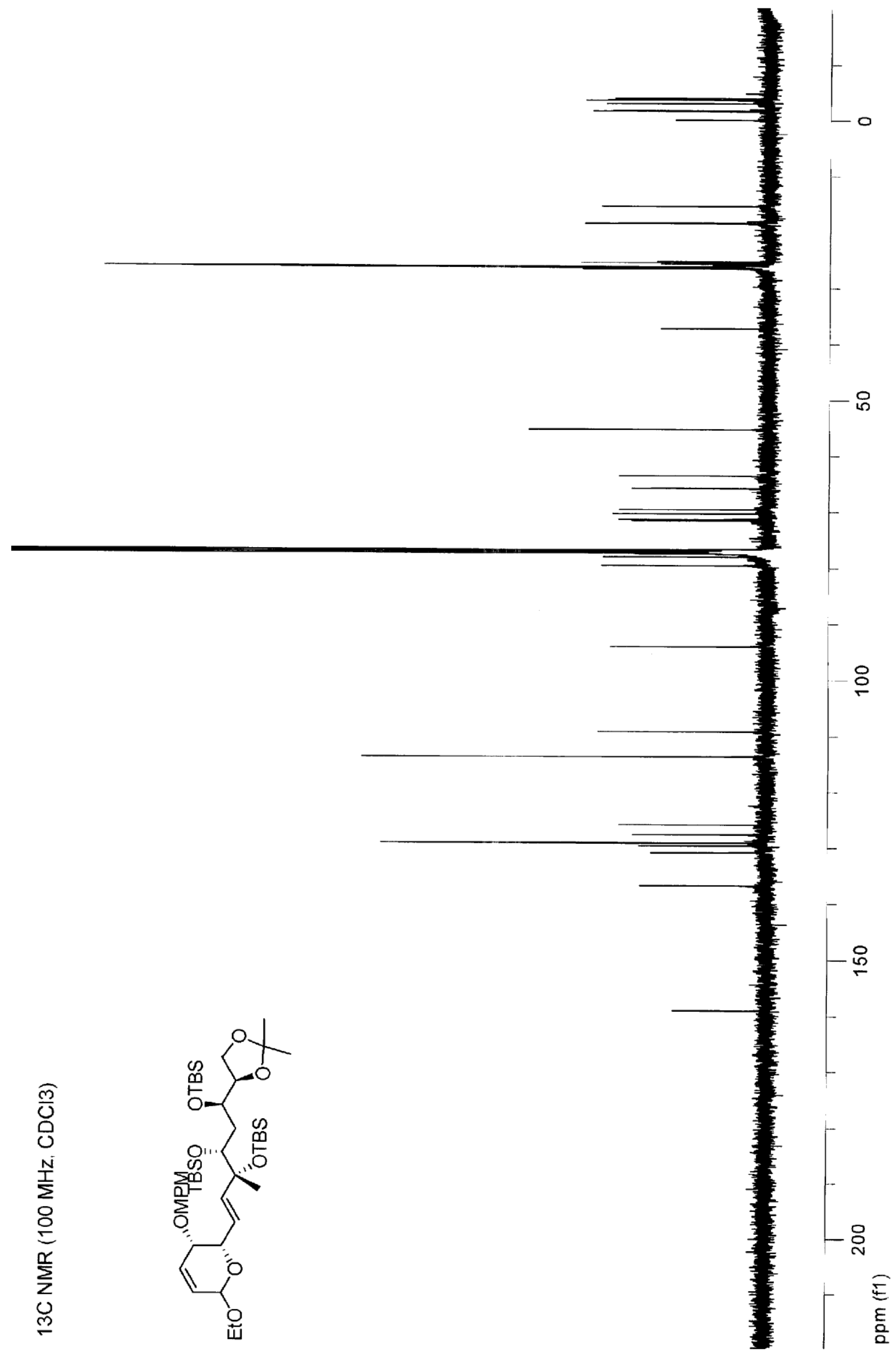




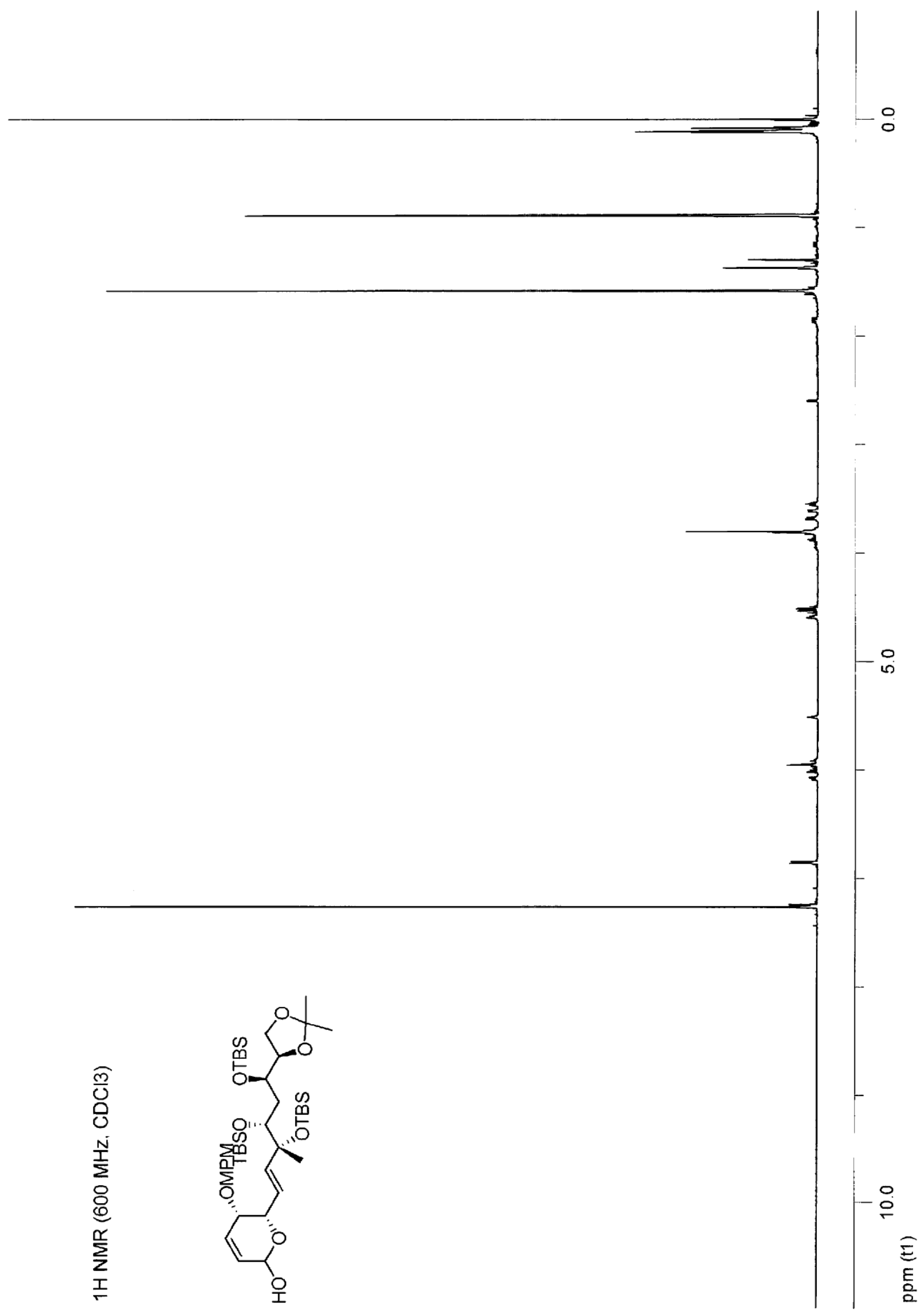




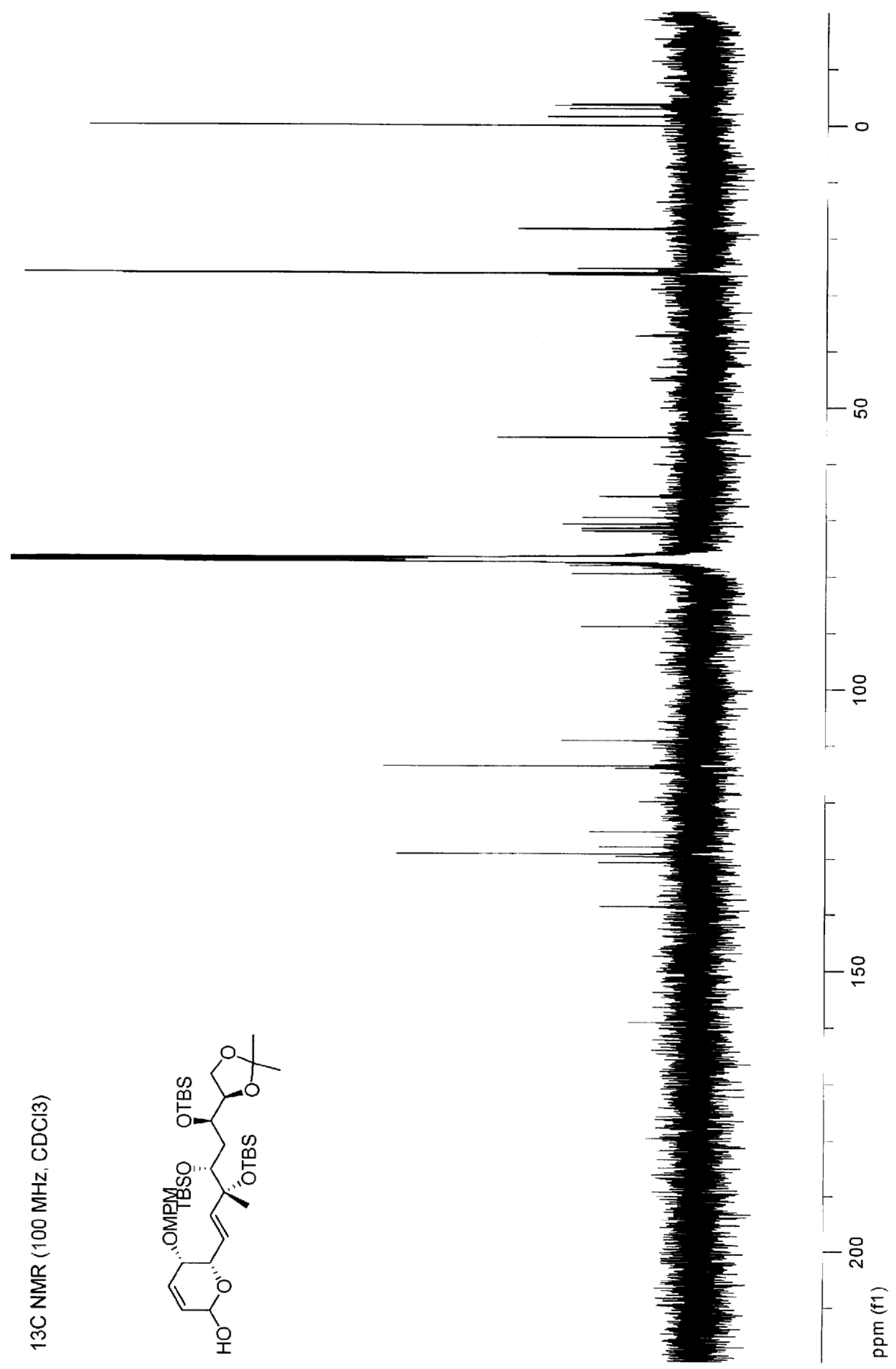




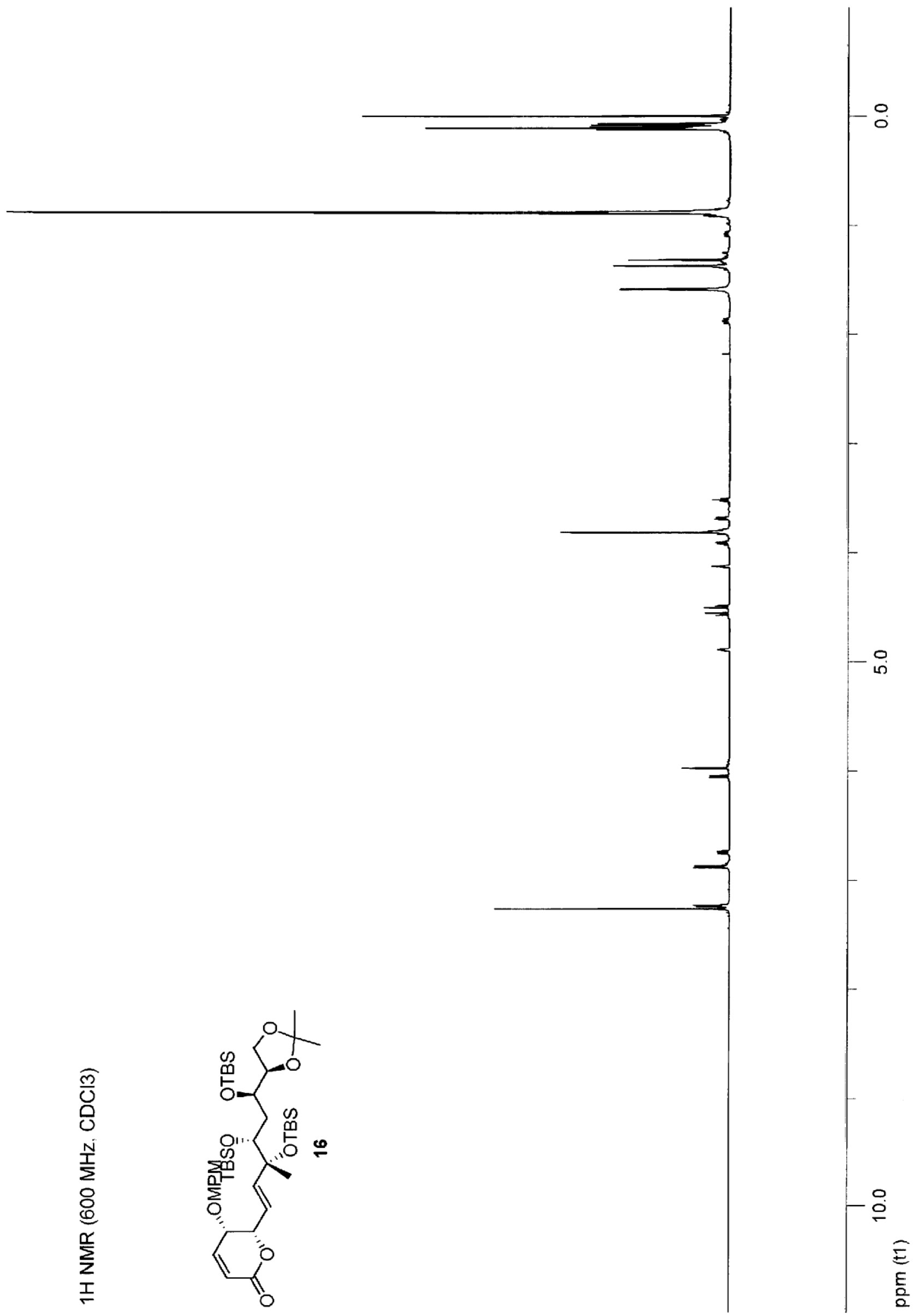




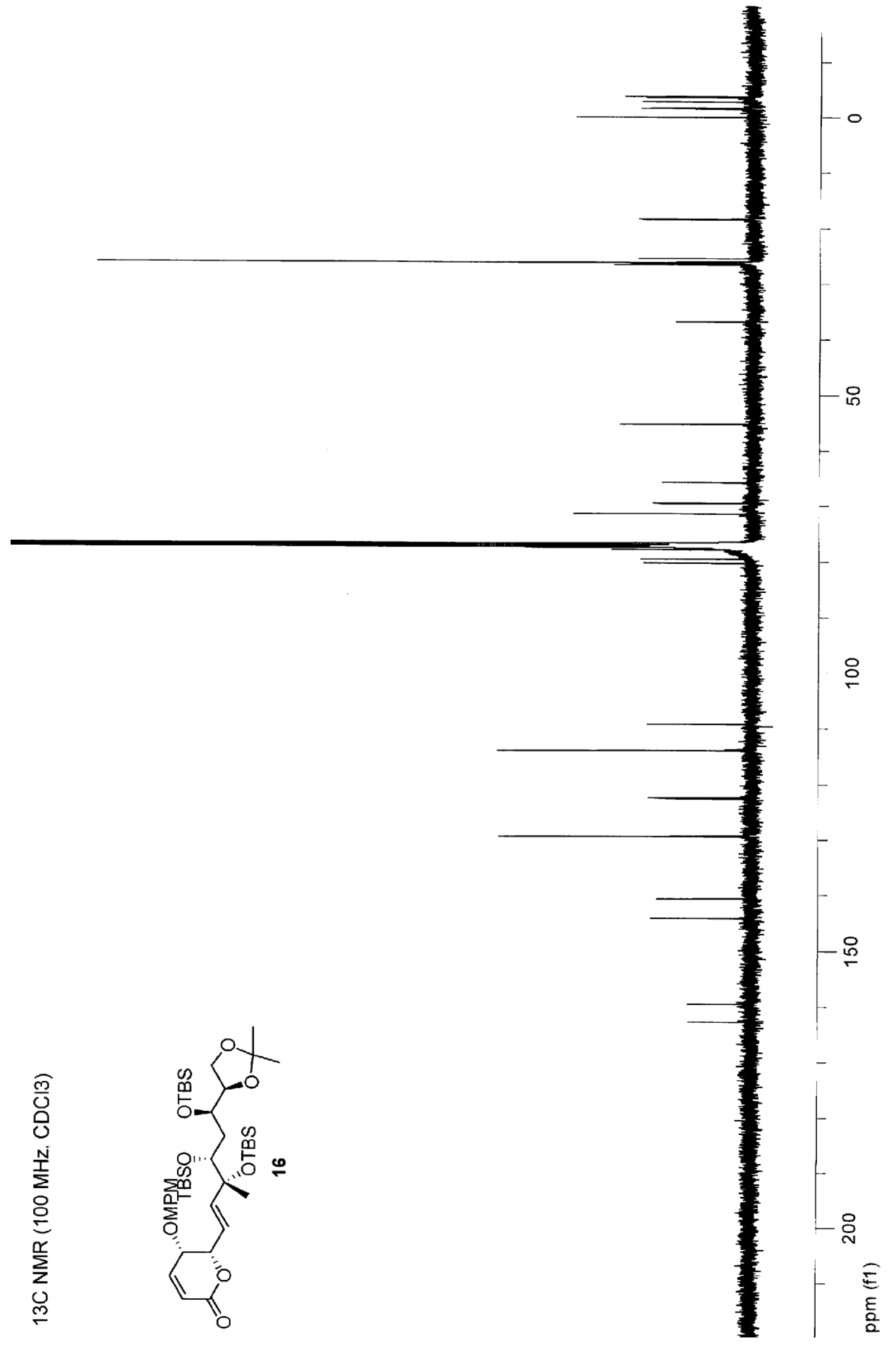




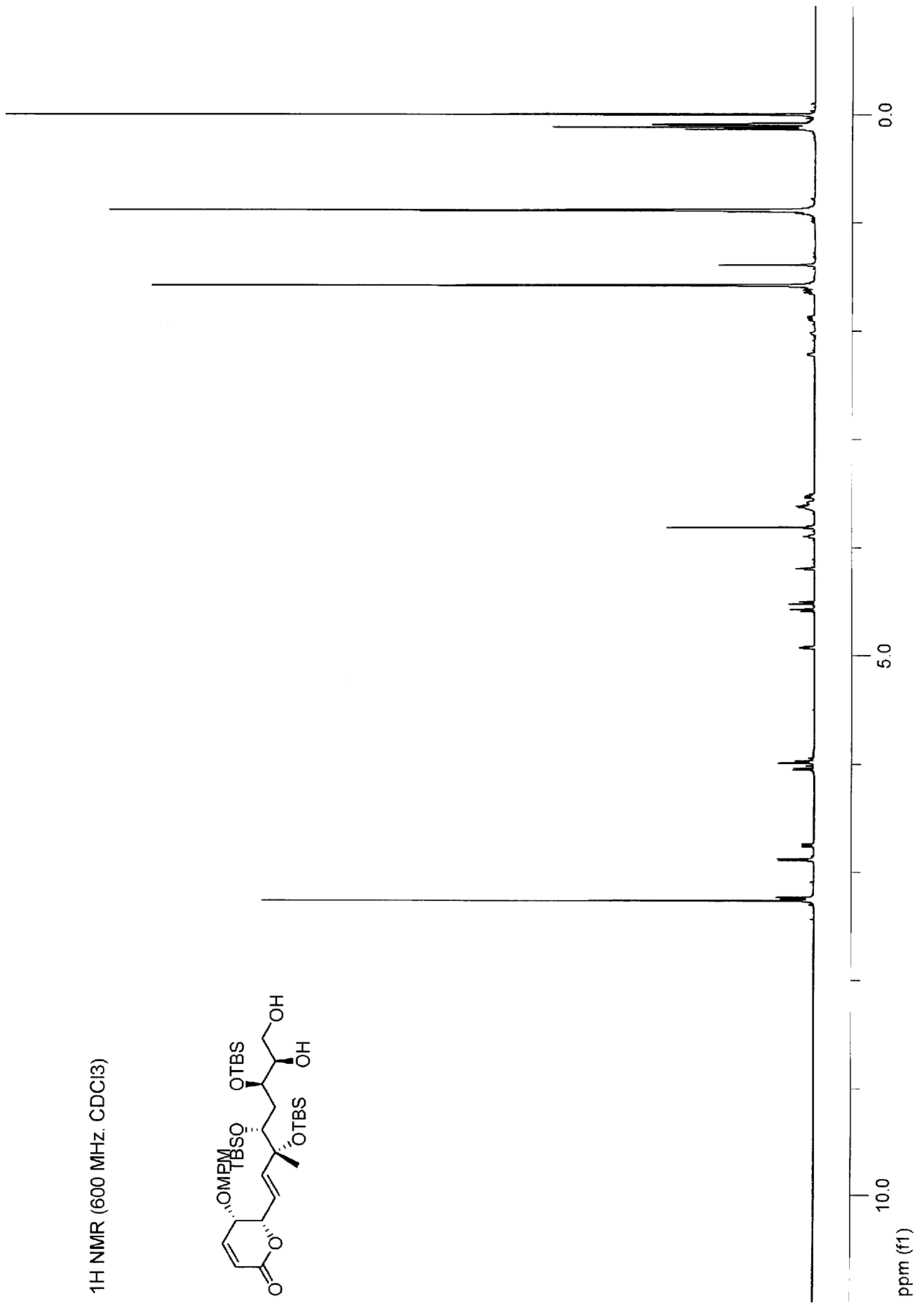




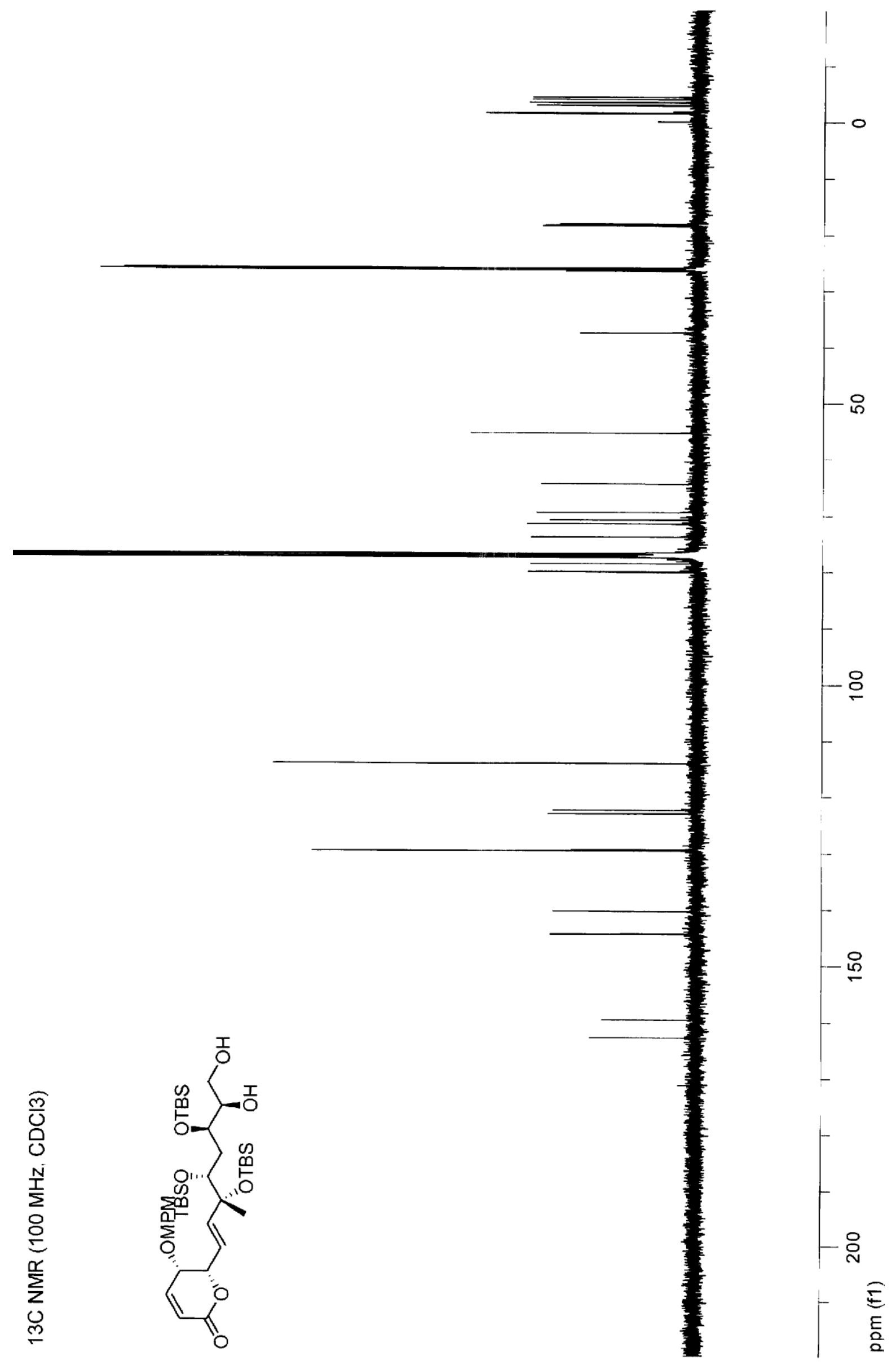




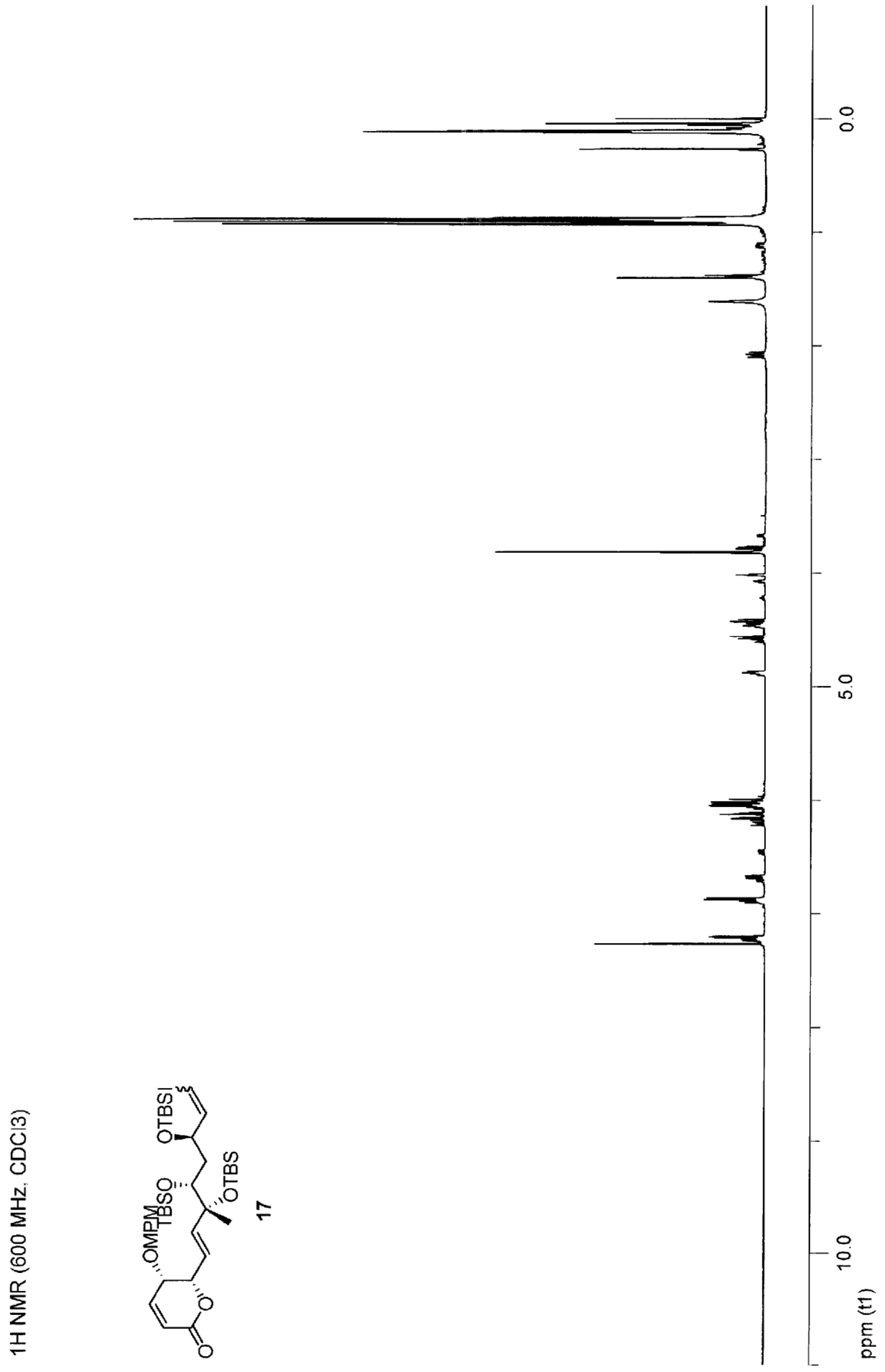





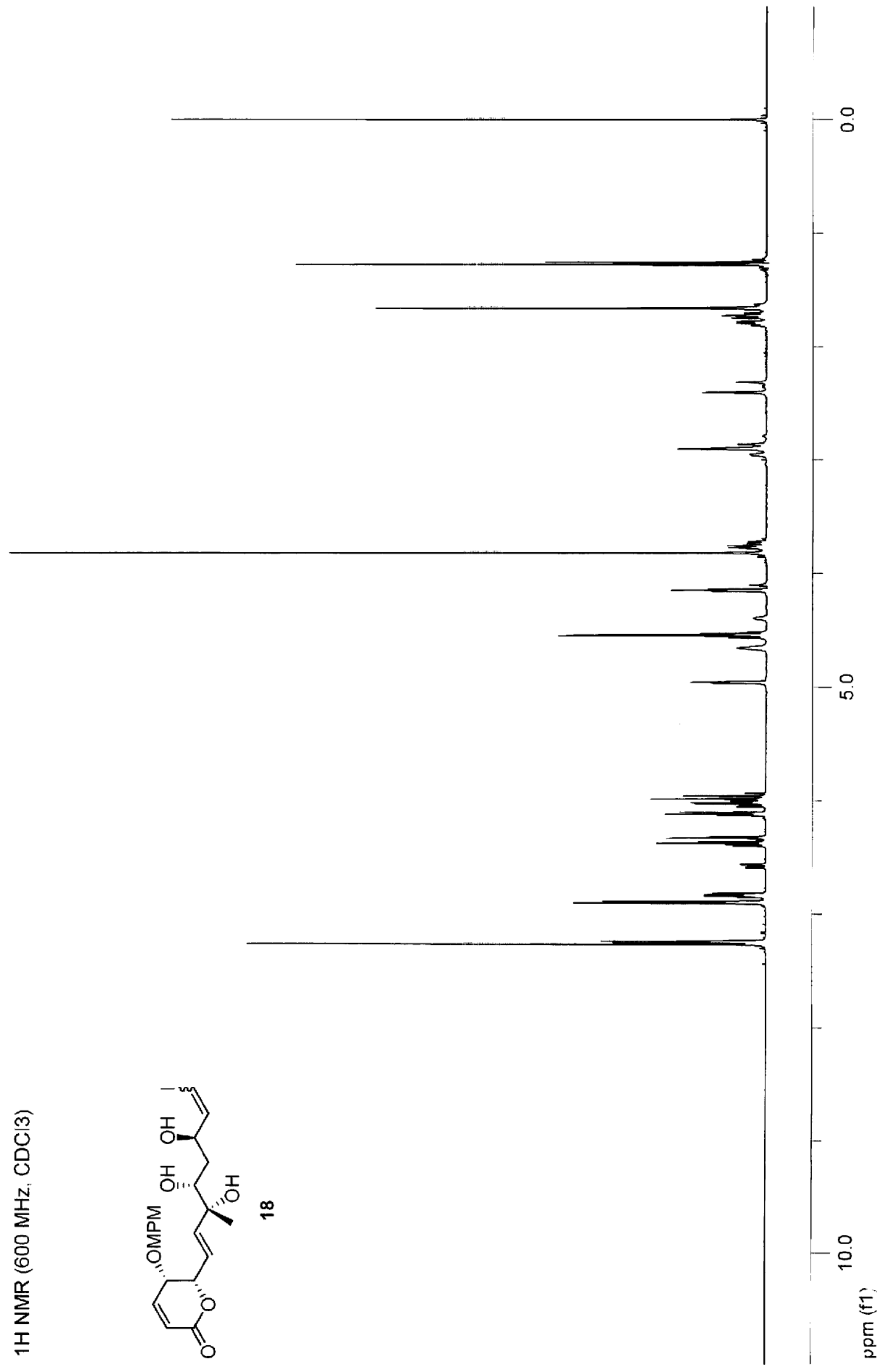




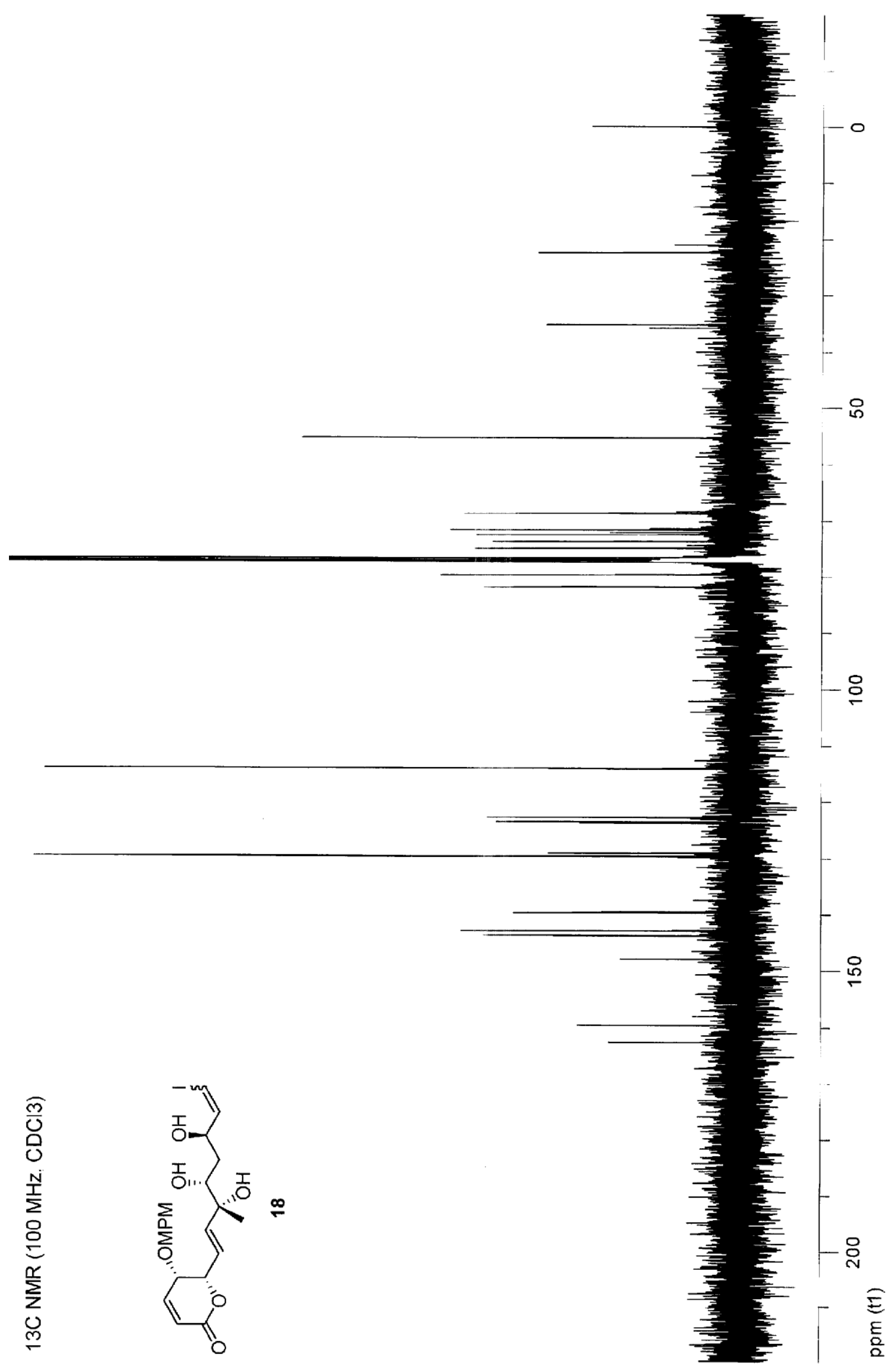




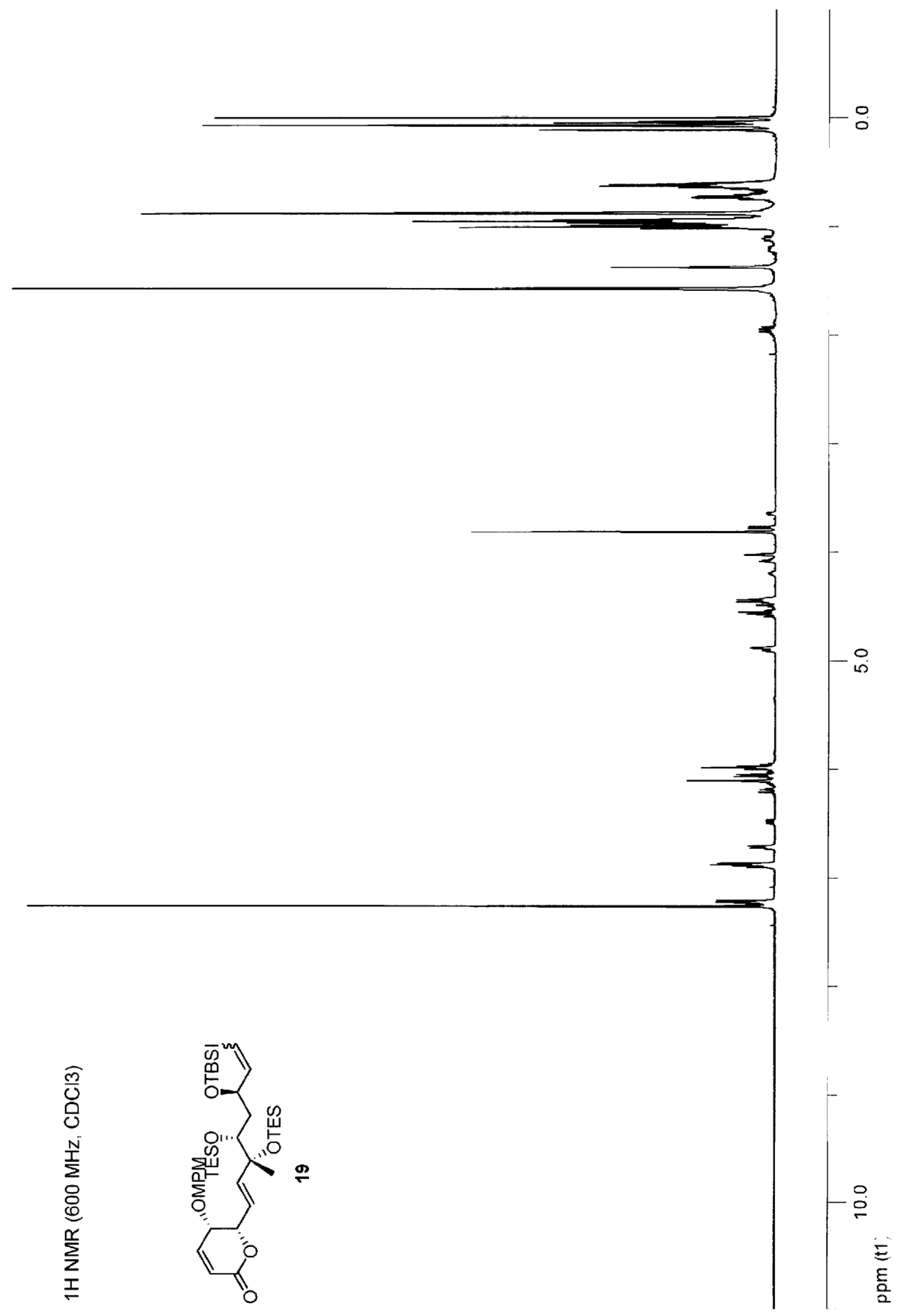




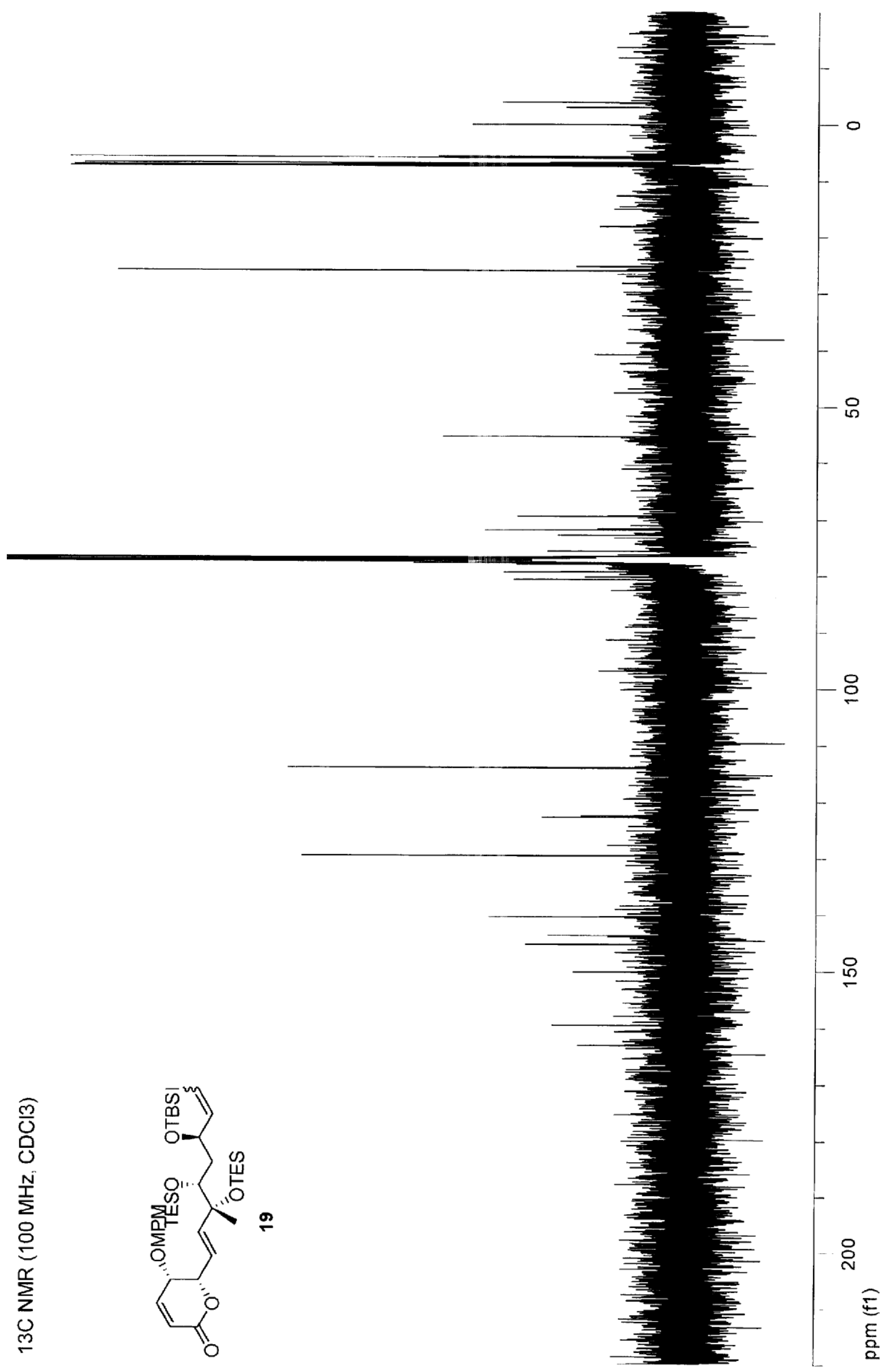




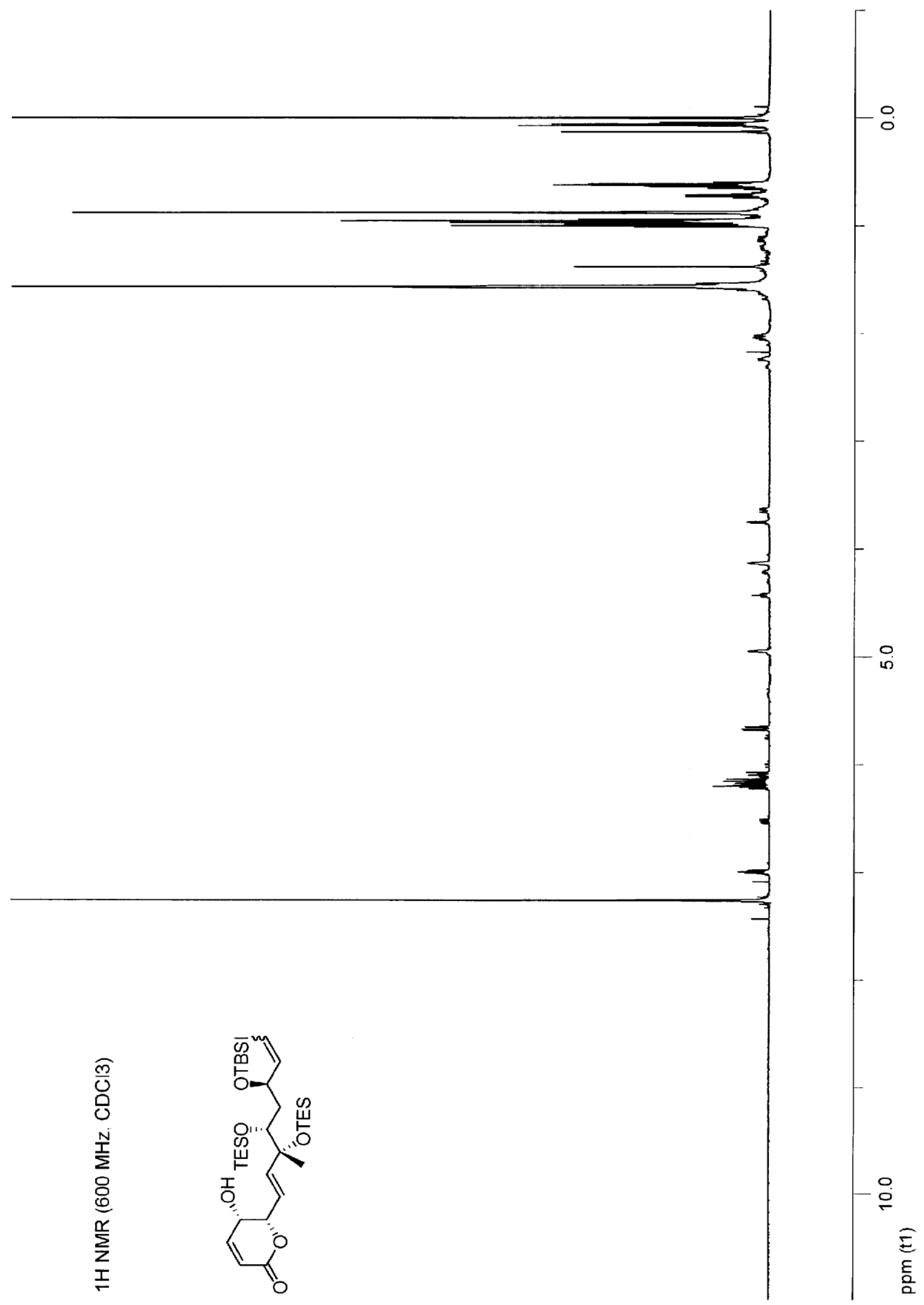




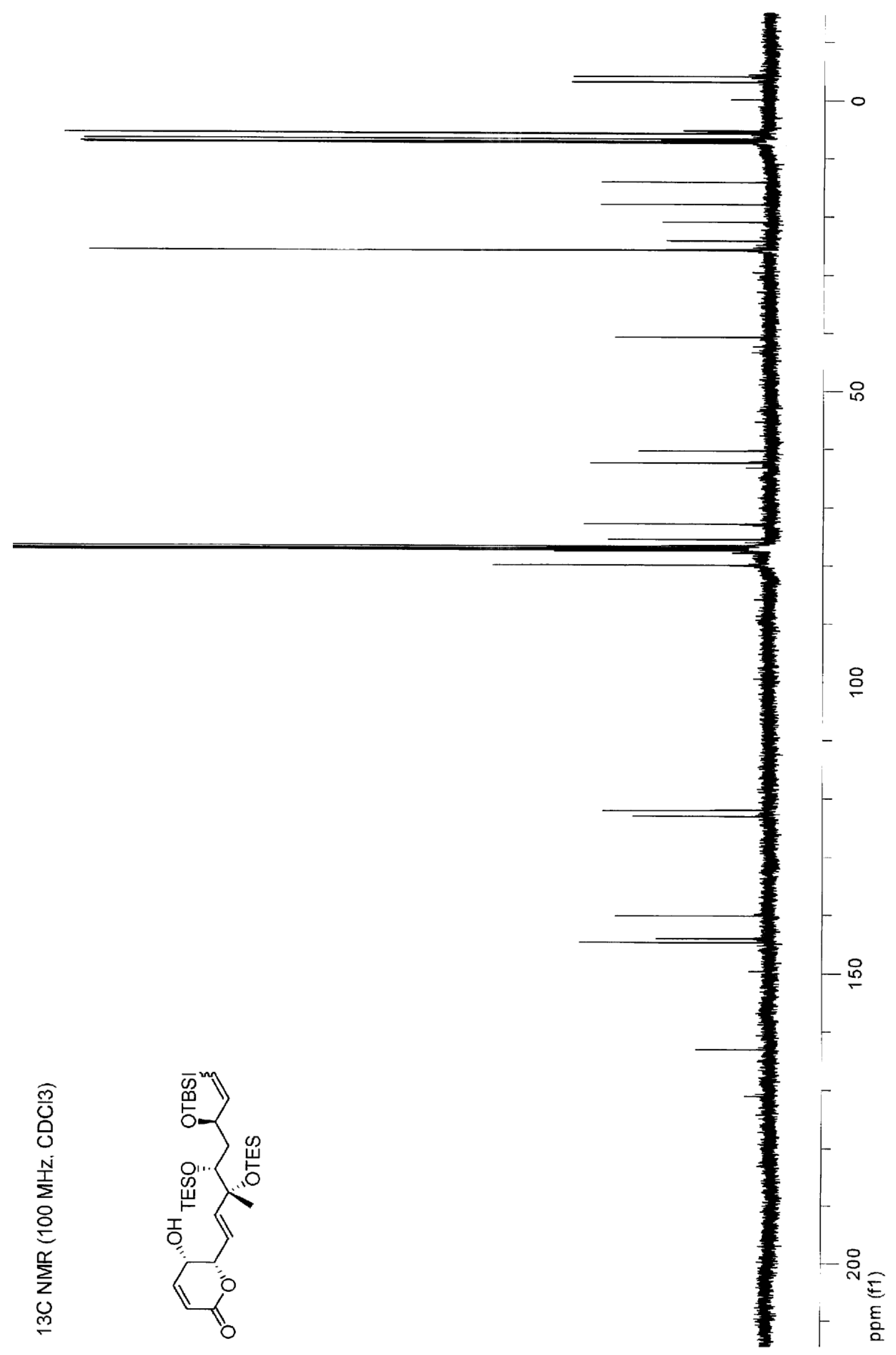




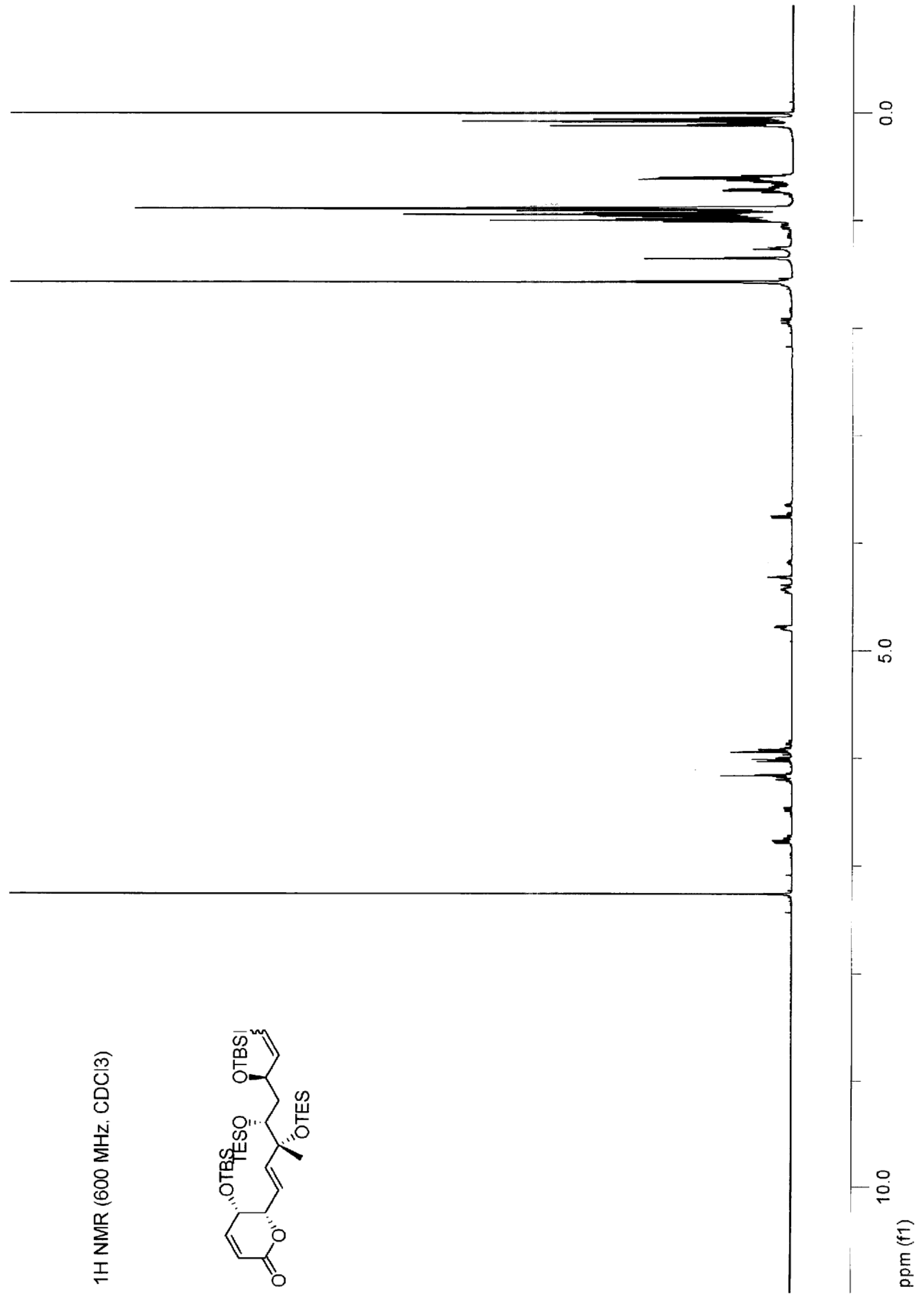




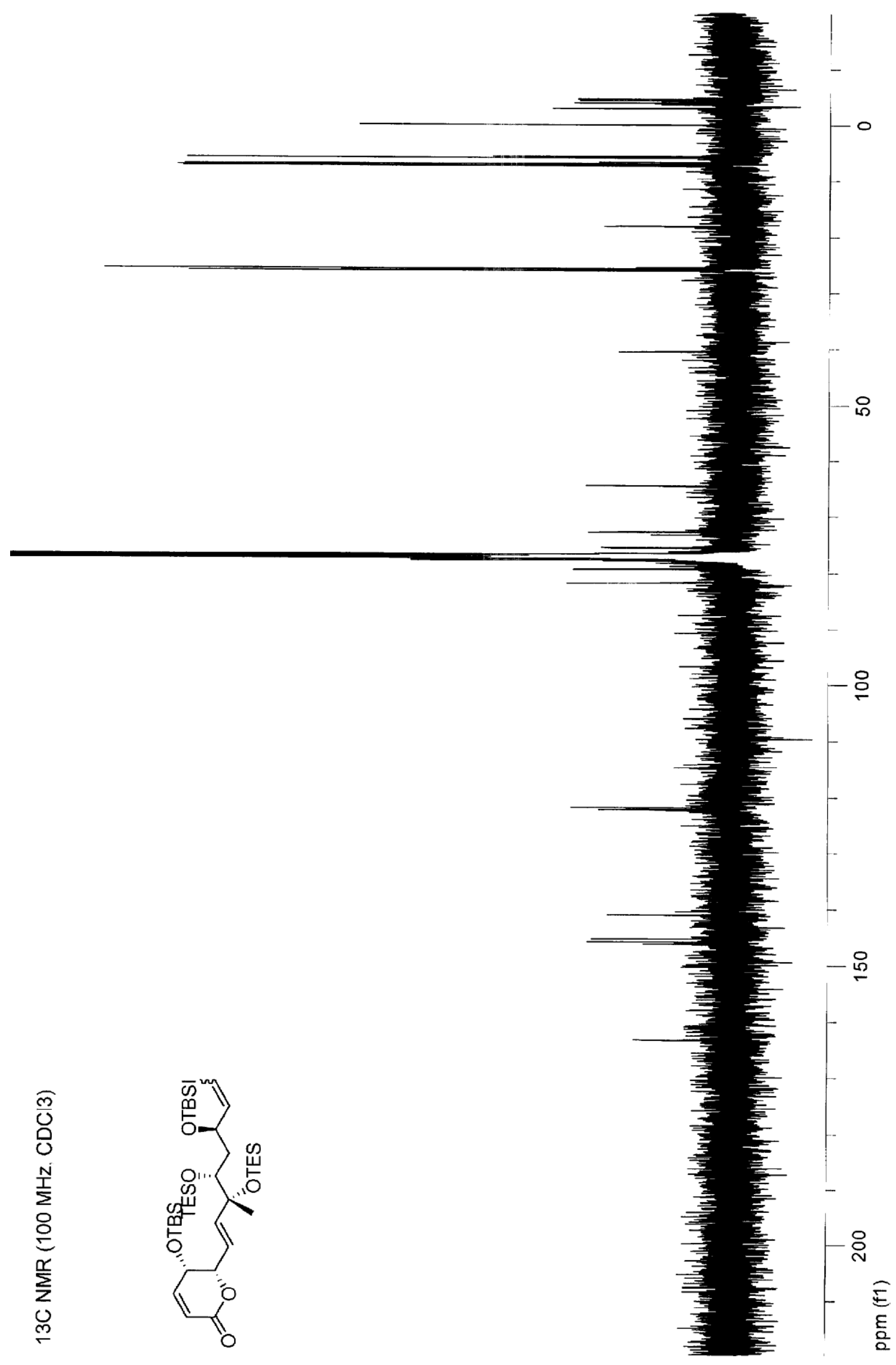




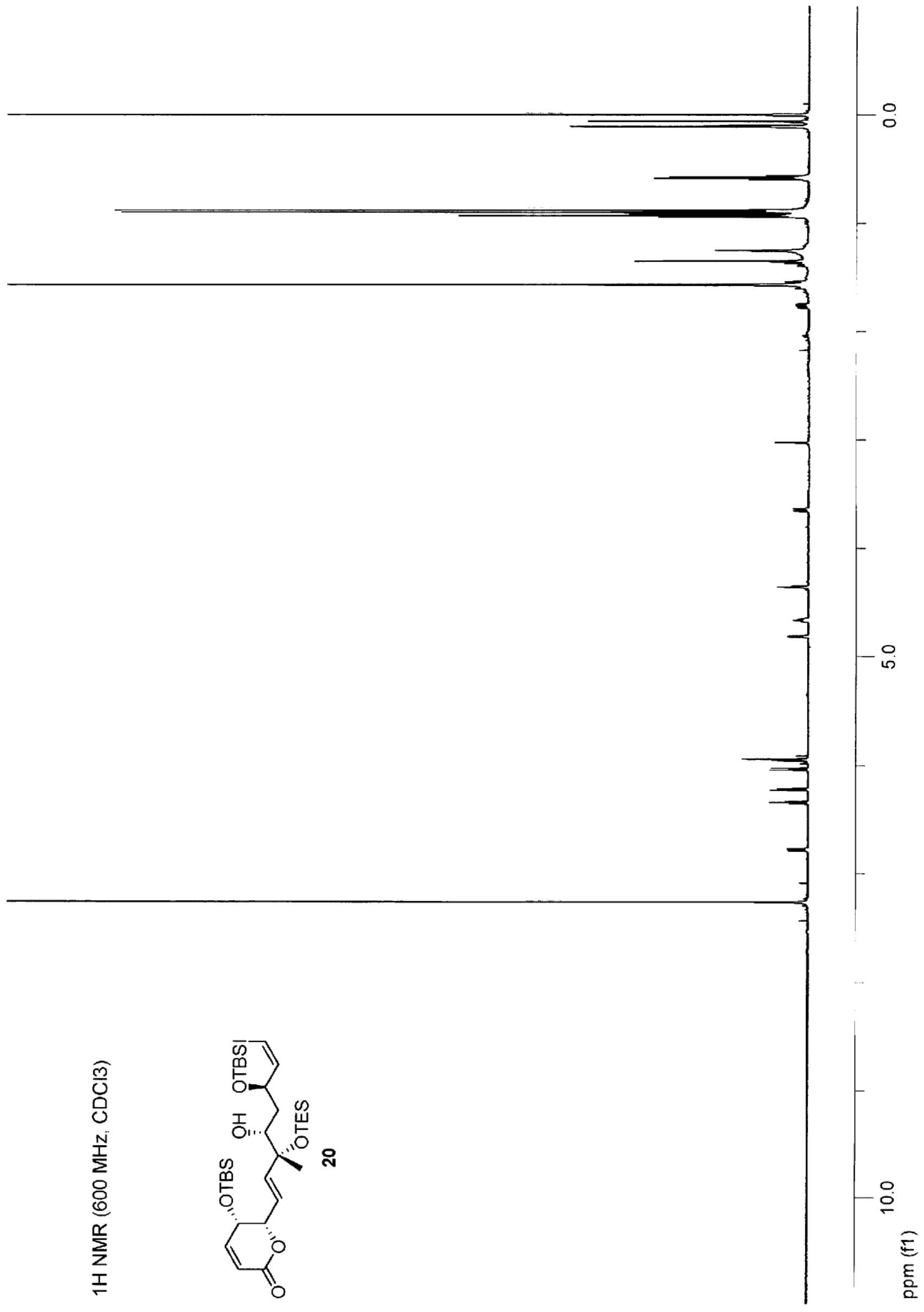




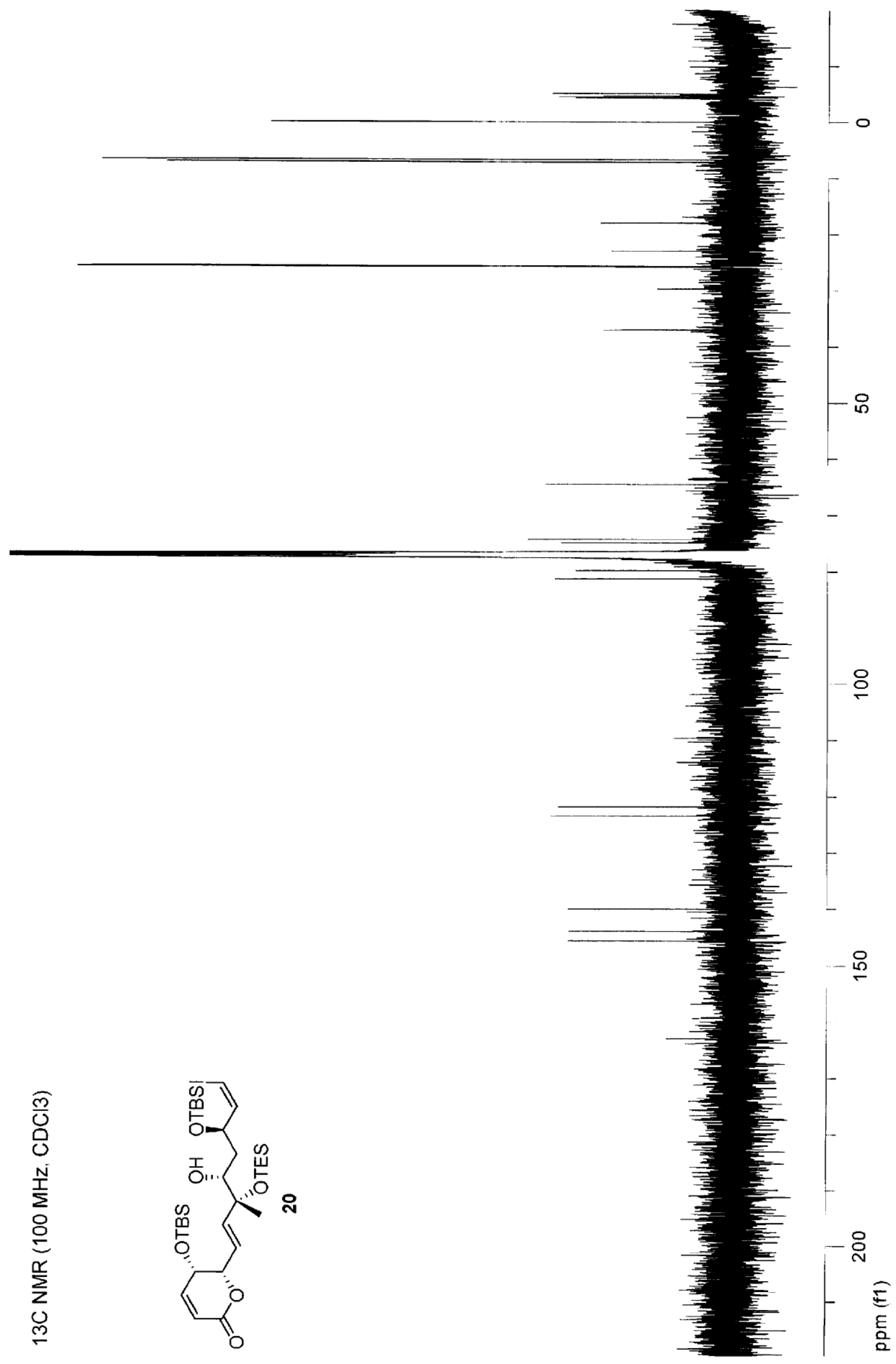




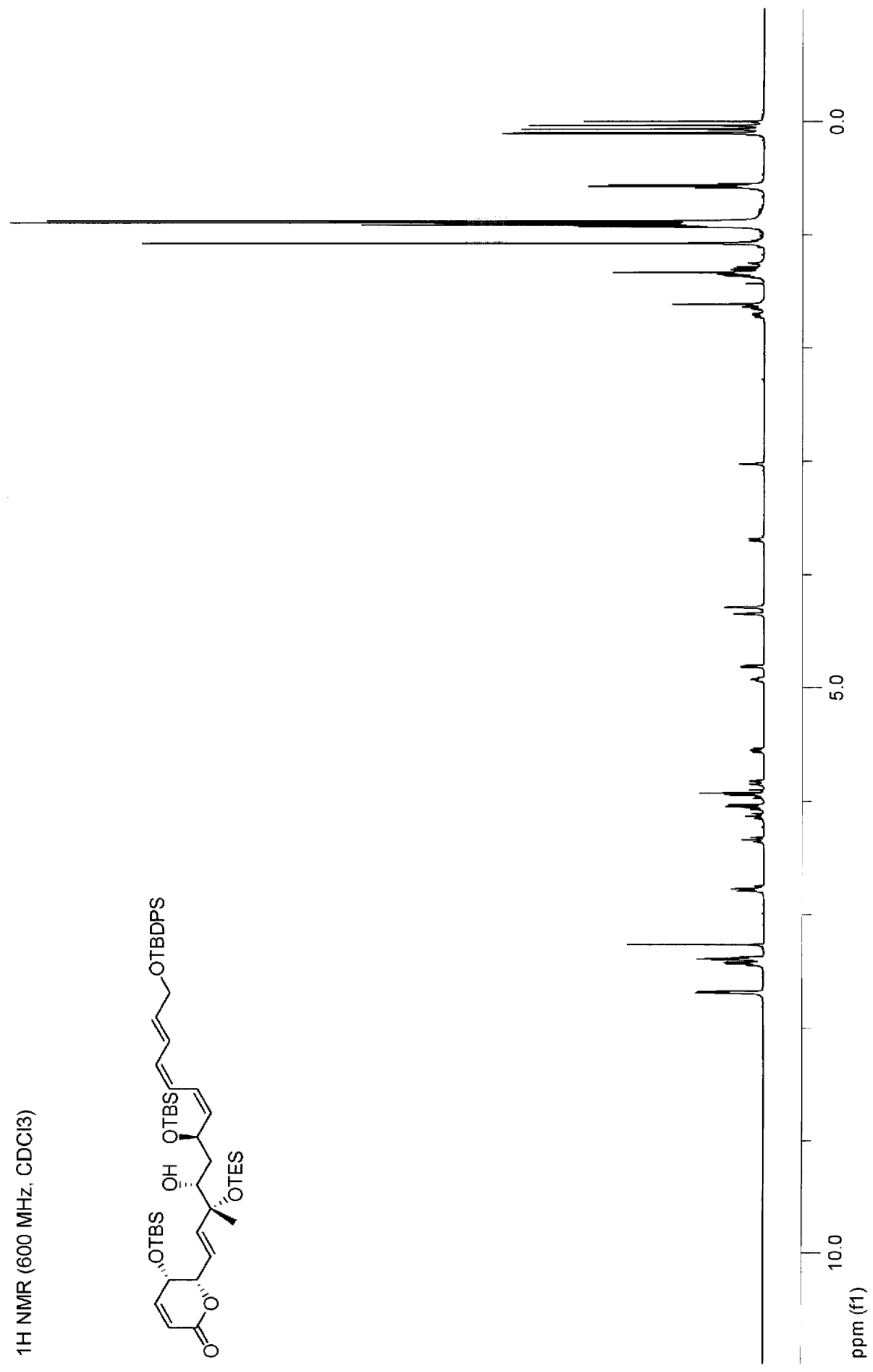




$$
\exists
$$




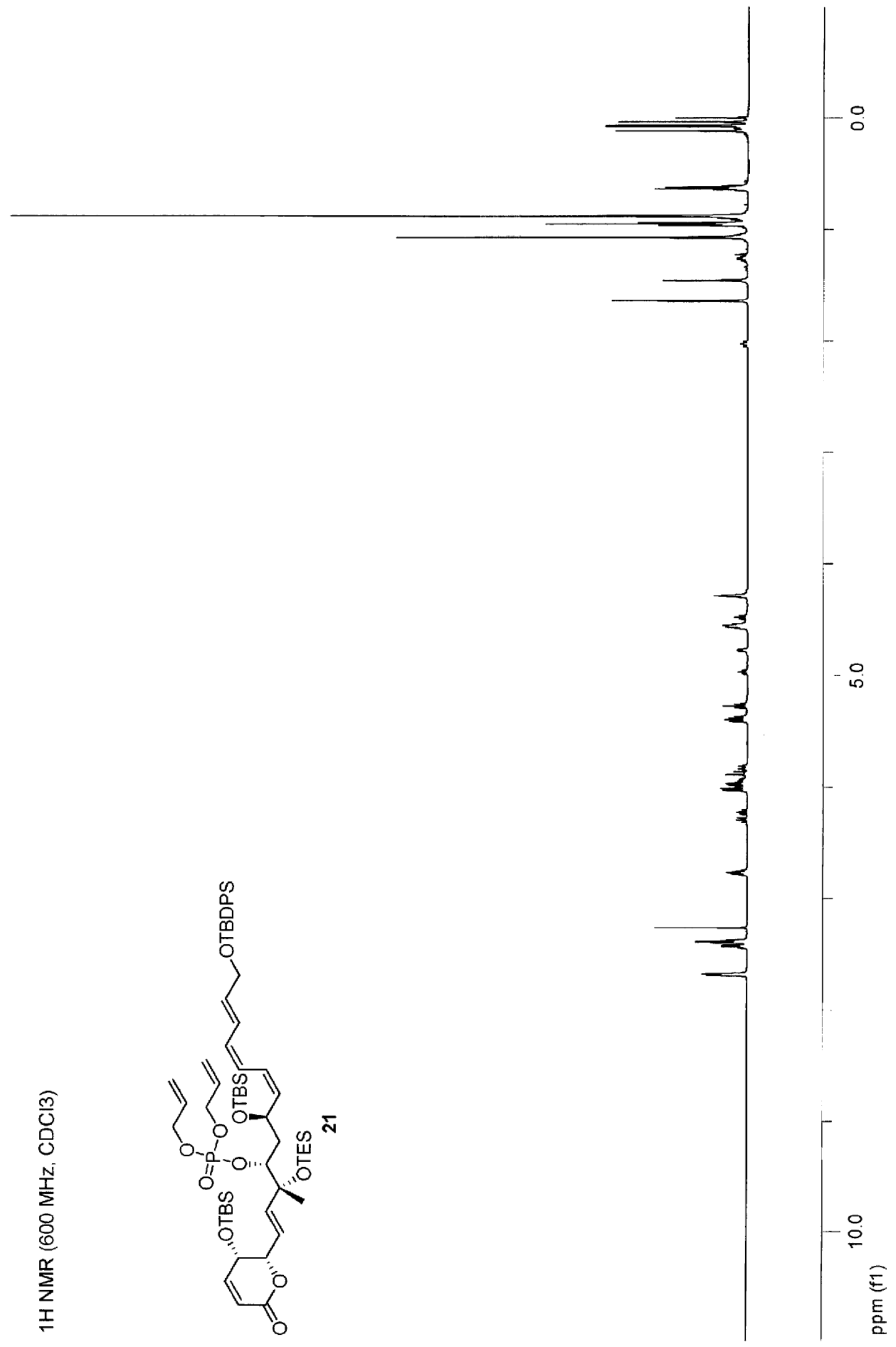




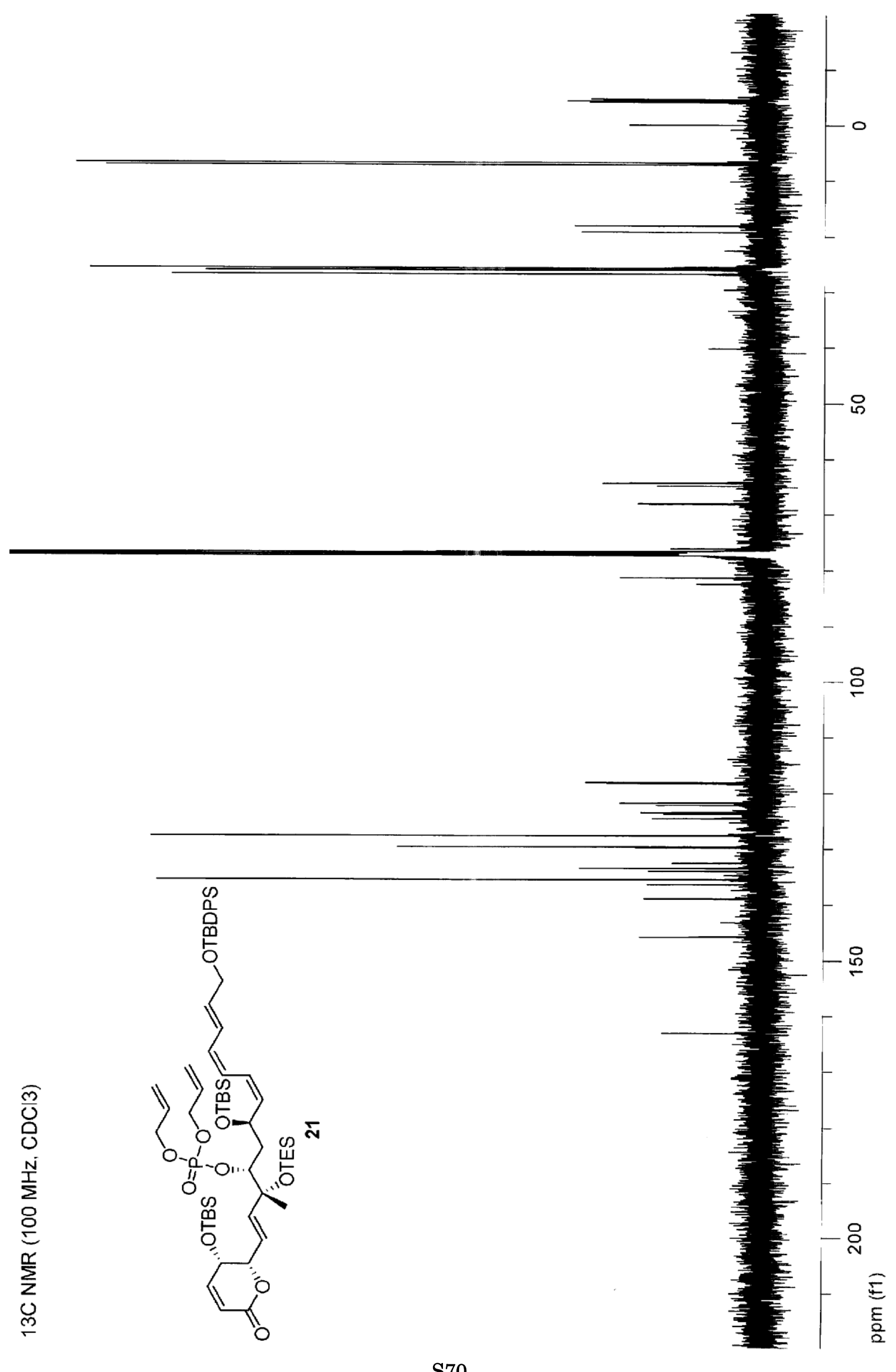

S70 


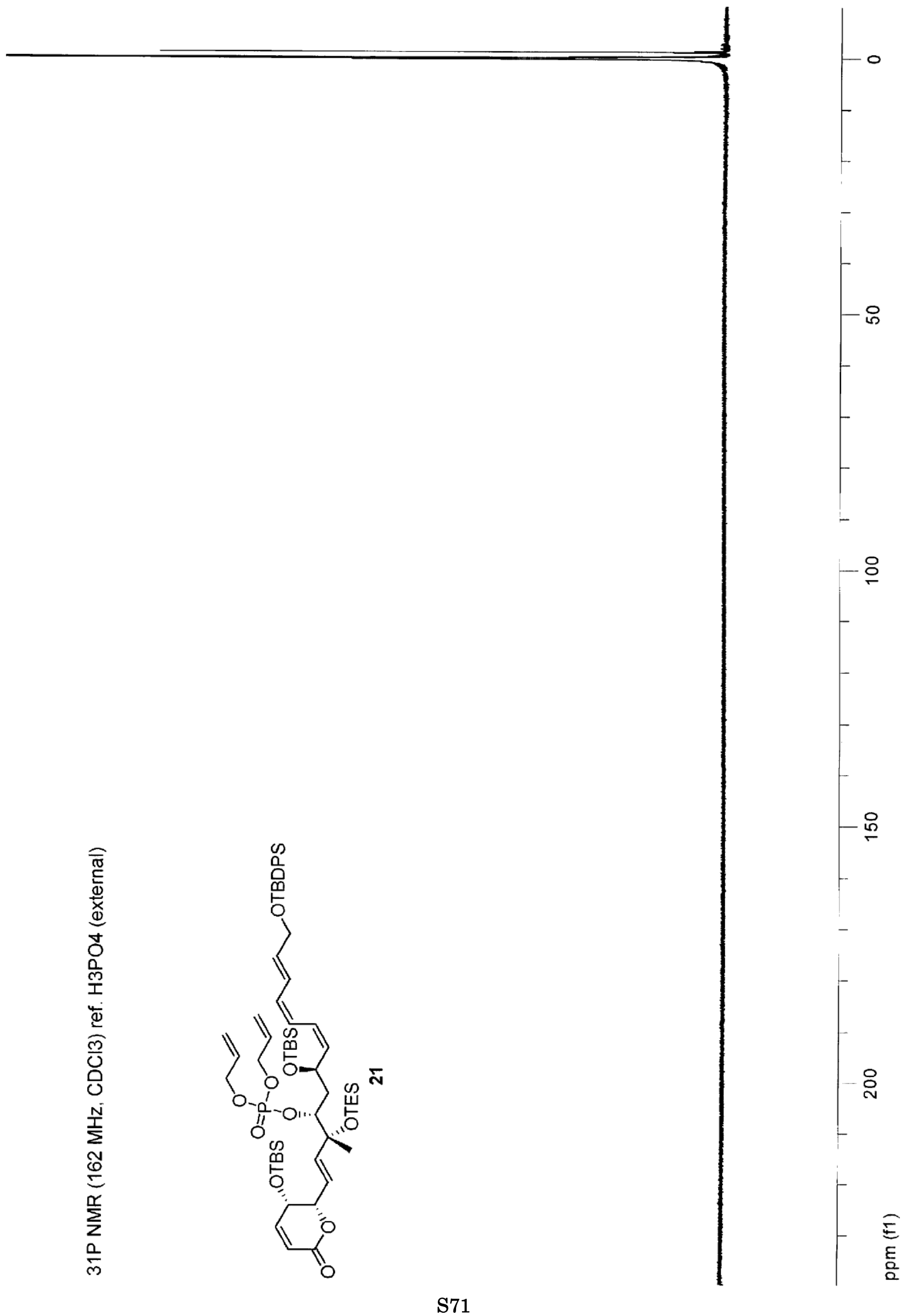




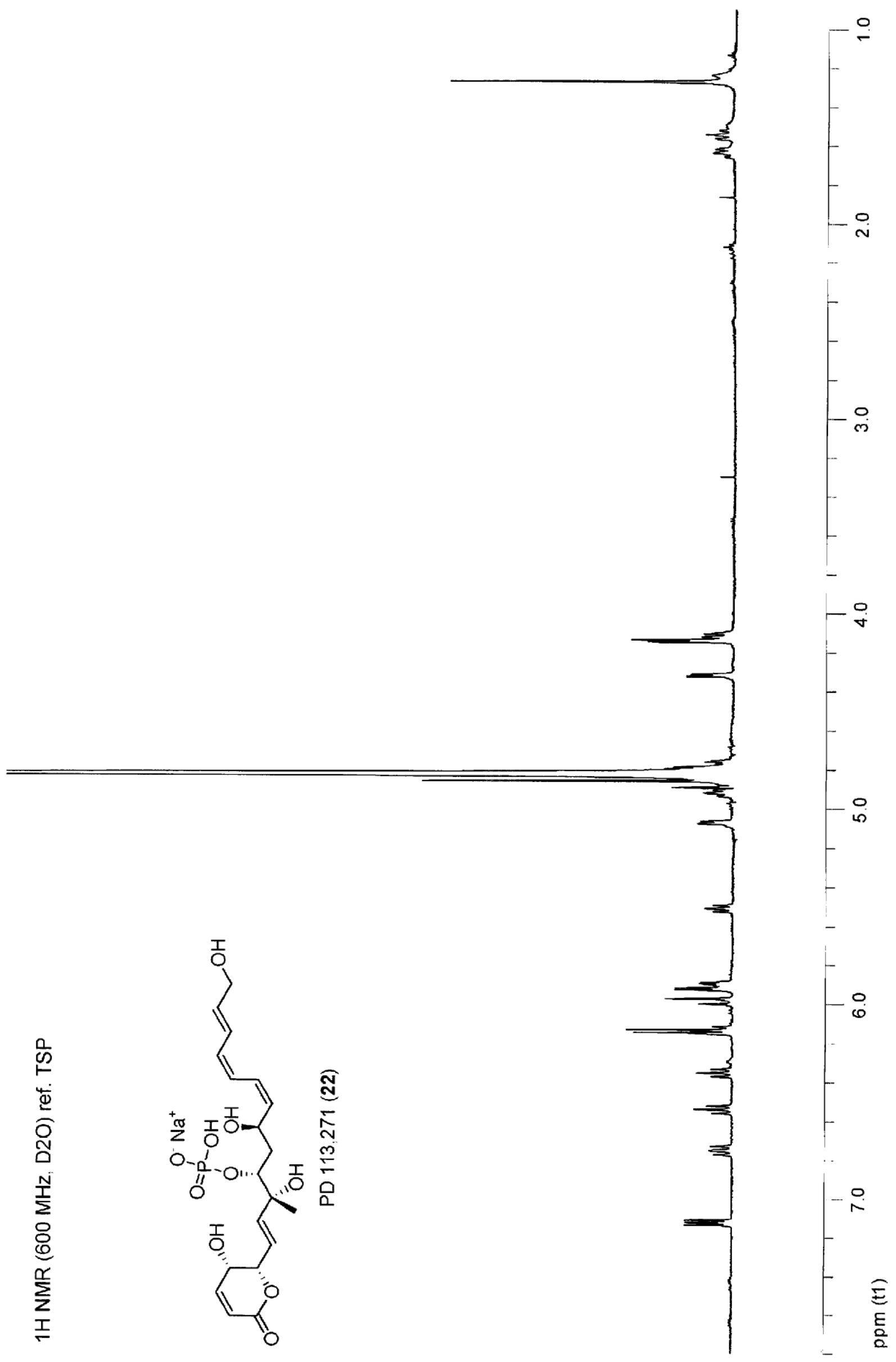




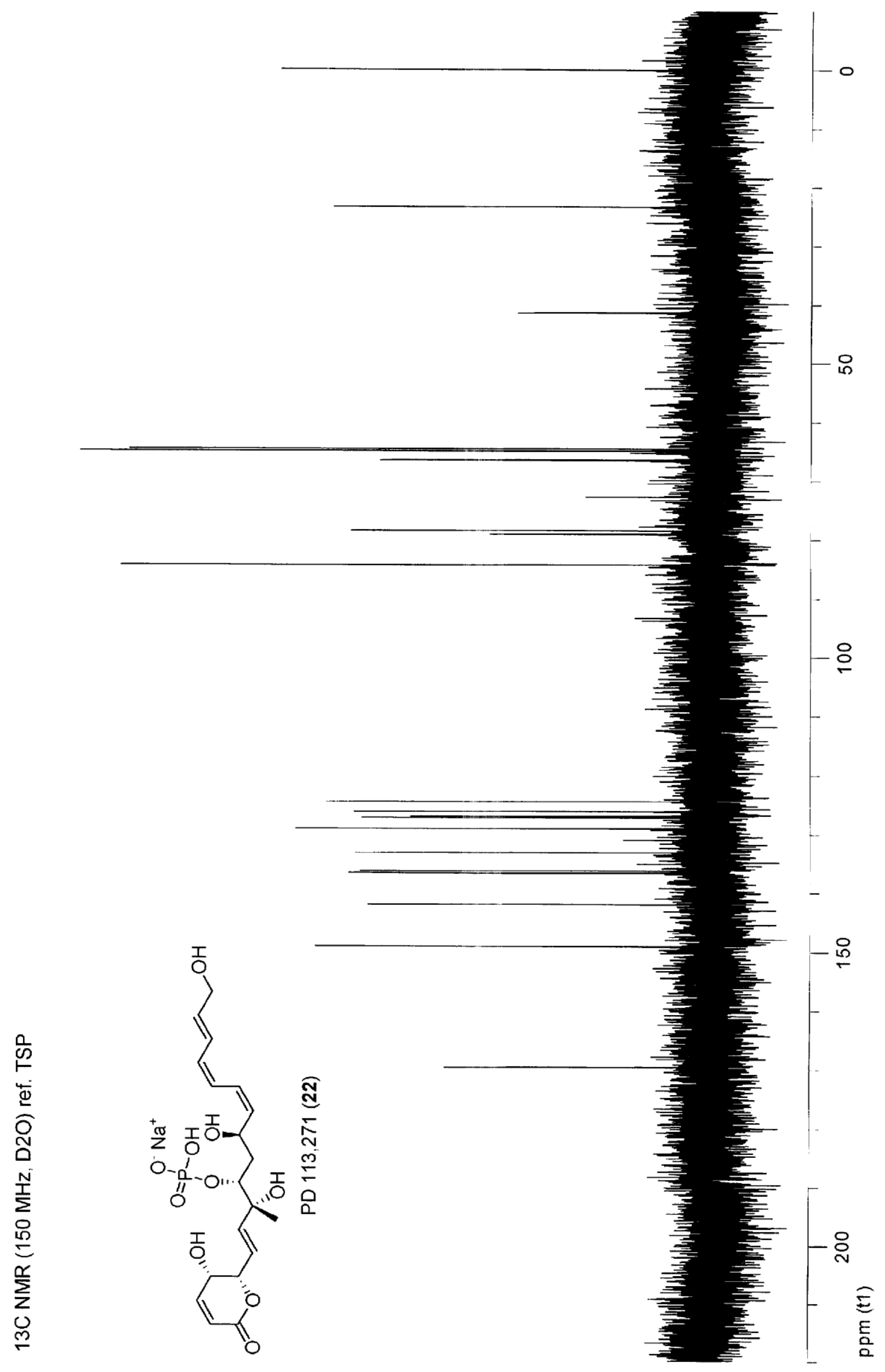




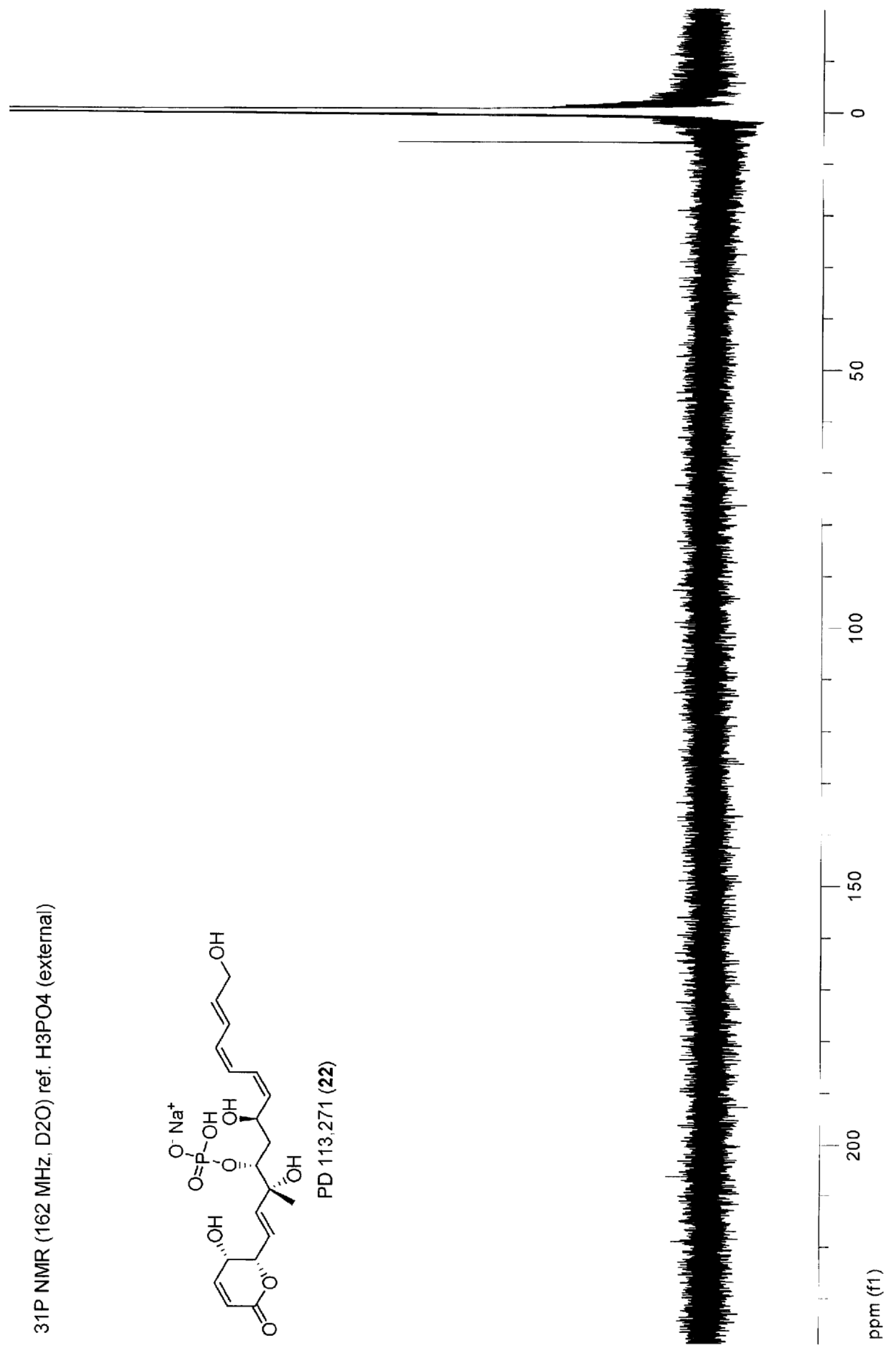




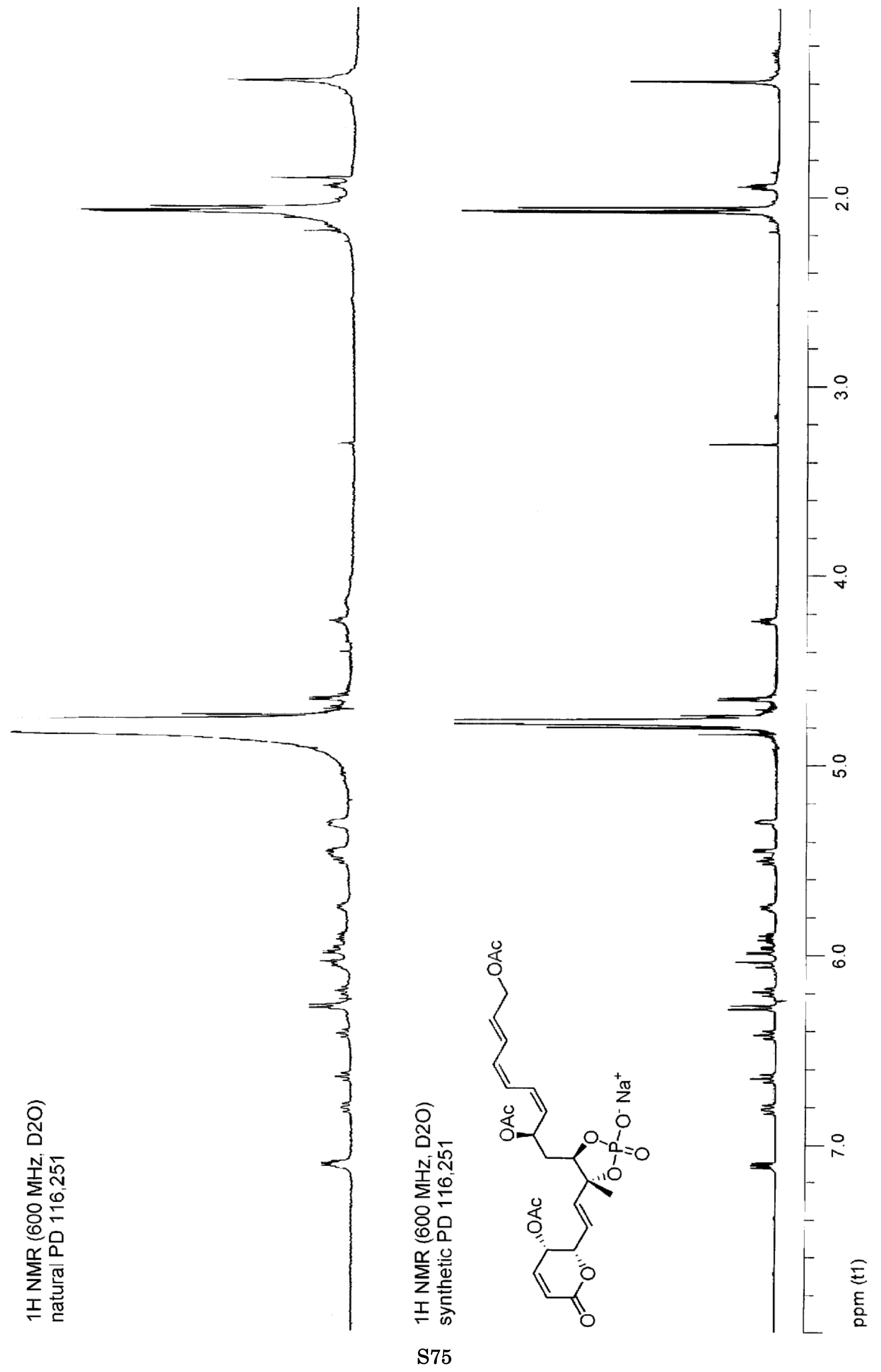




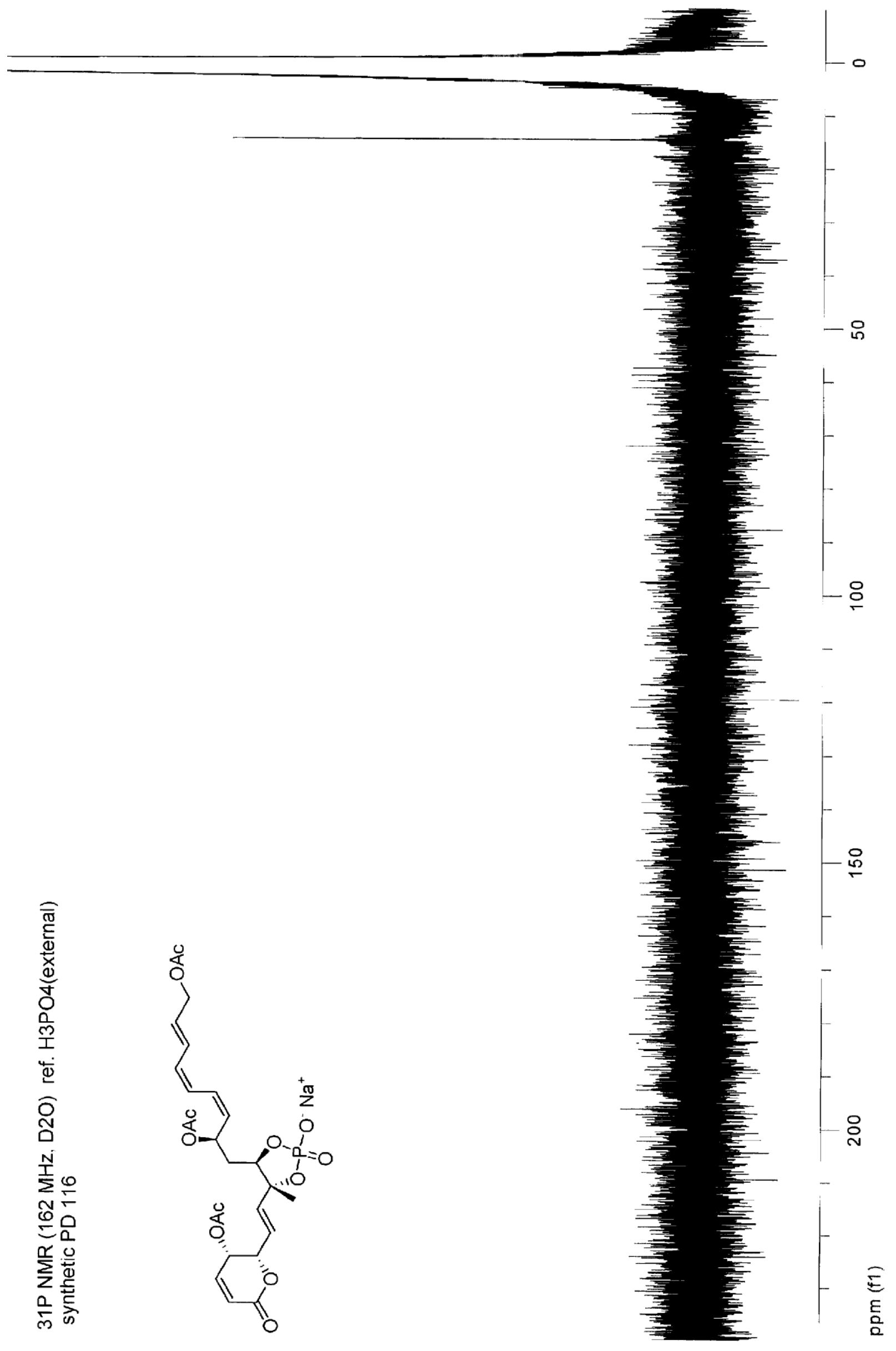




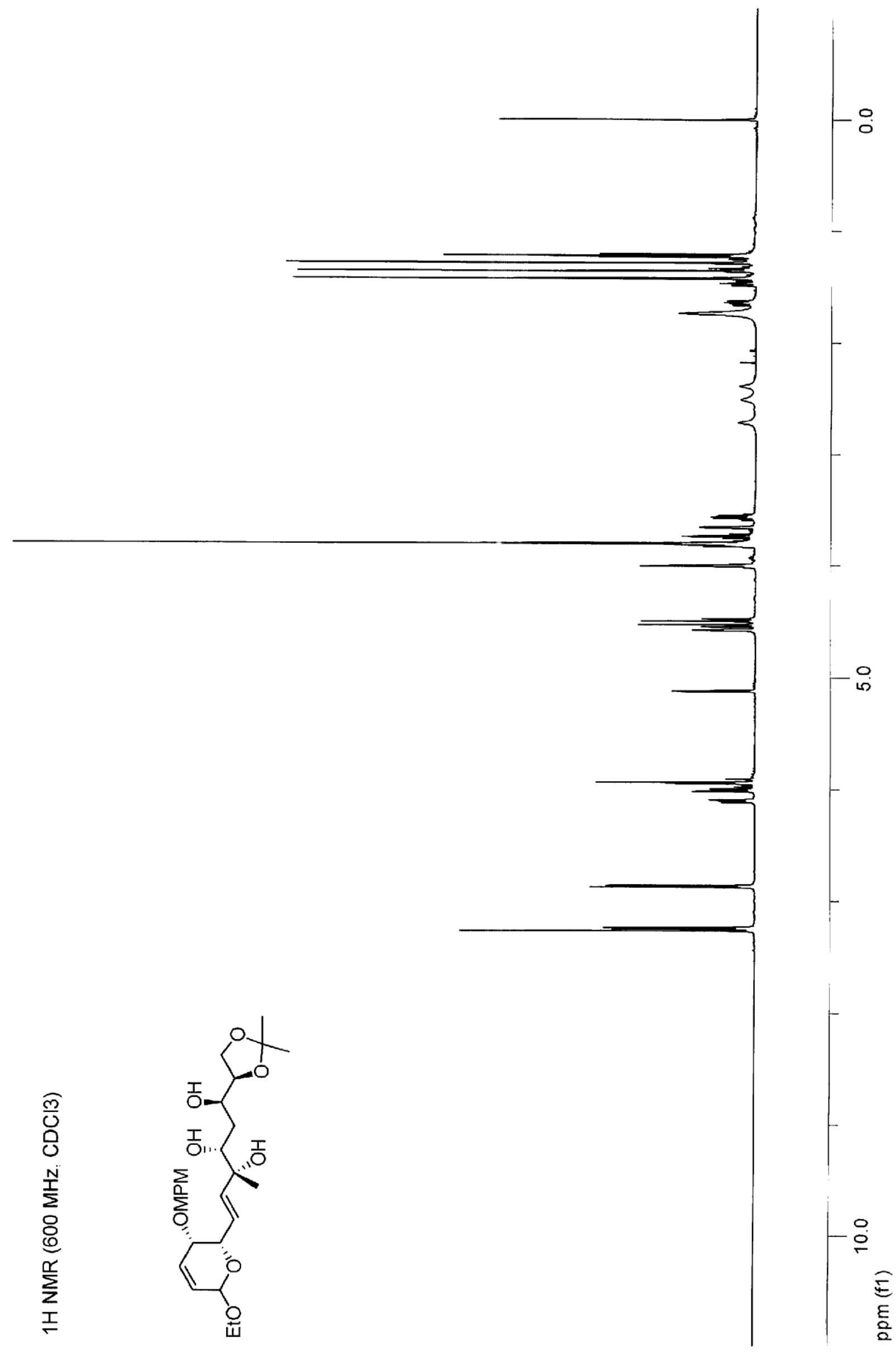




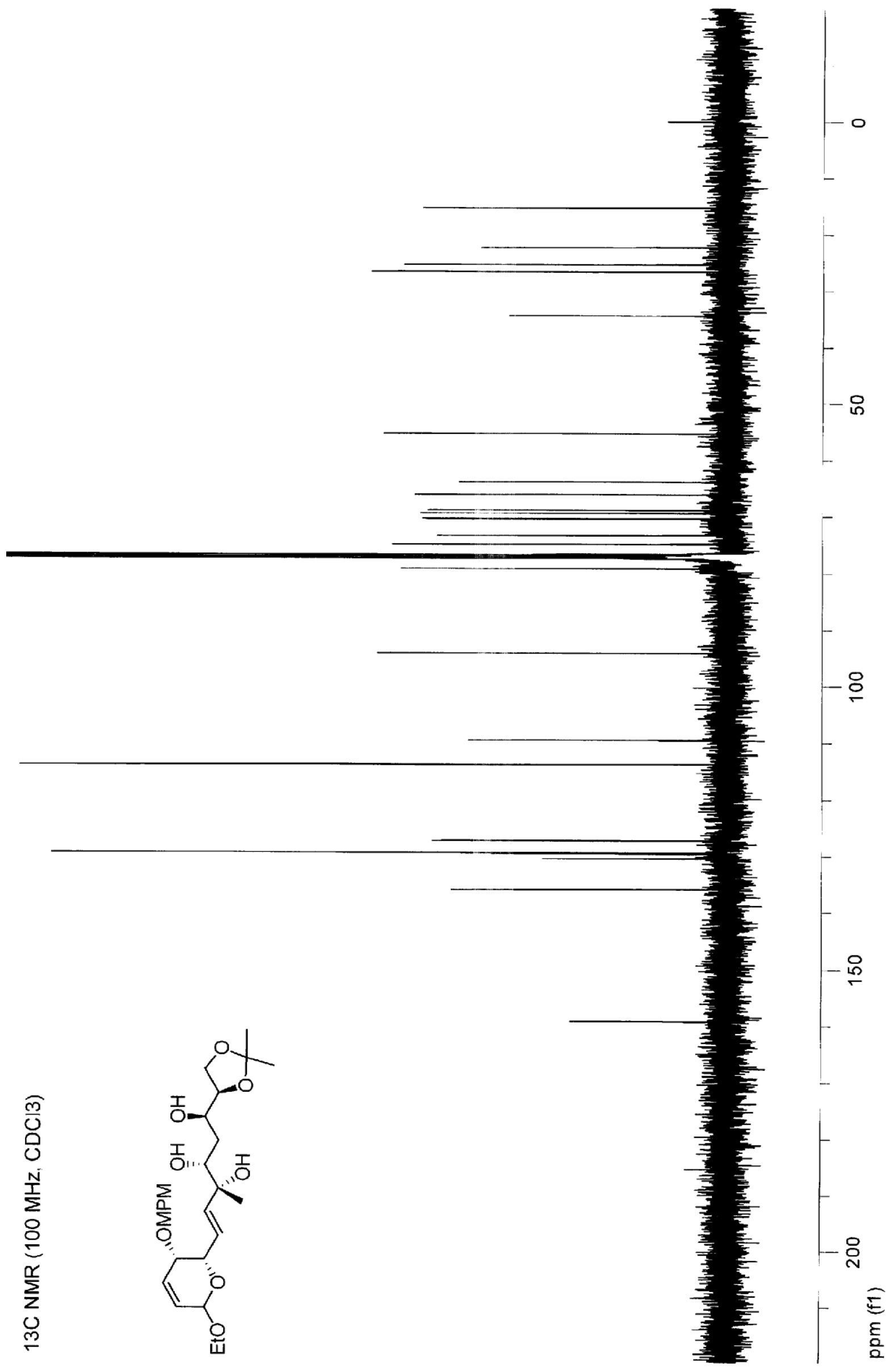




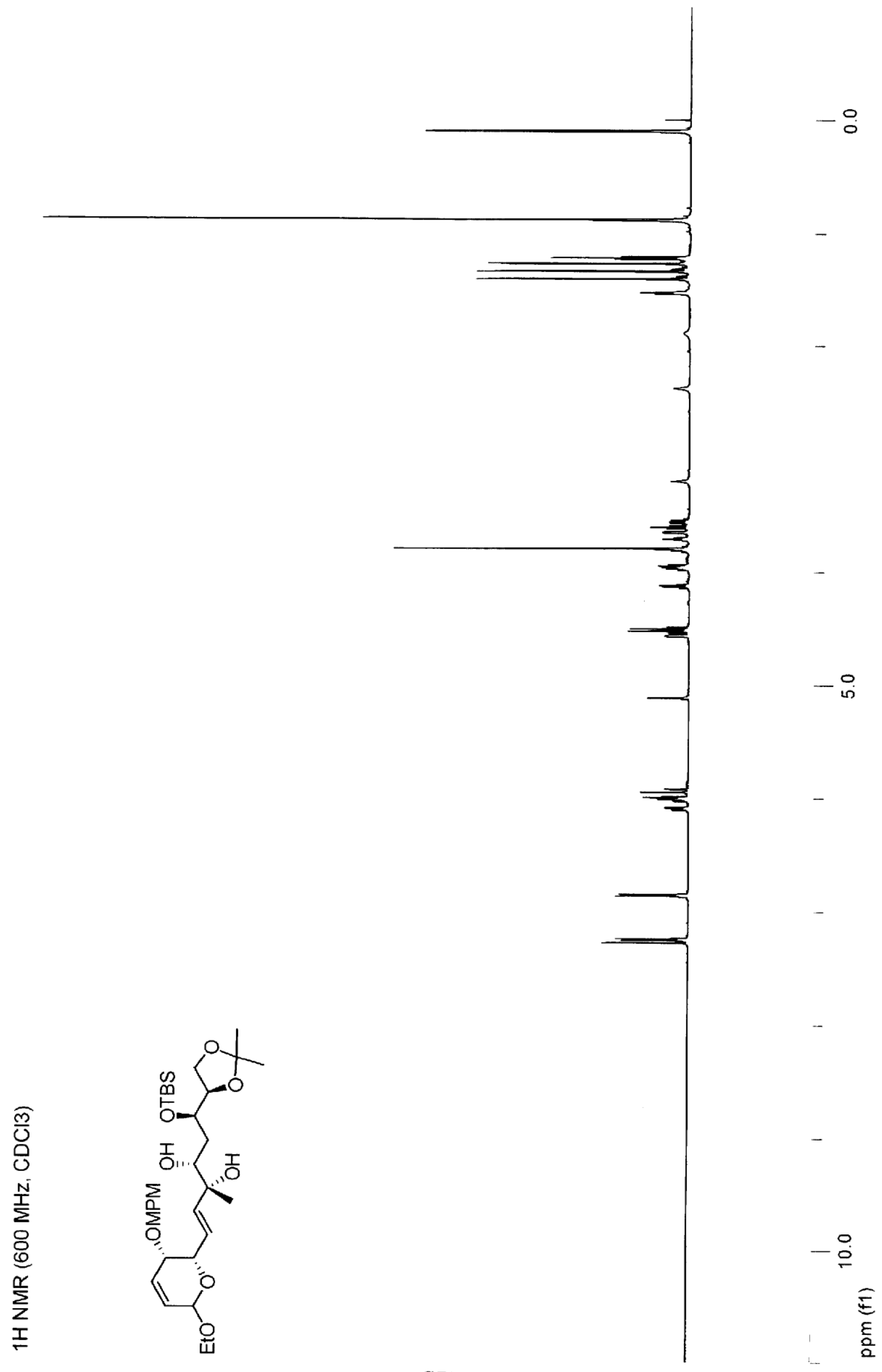




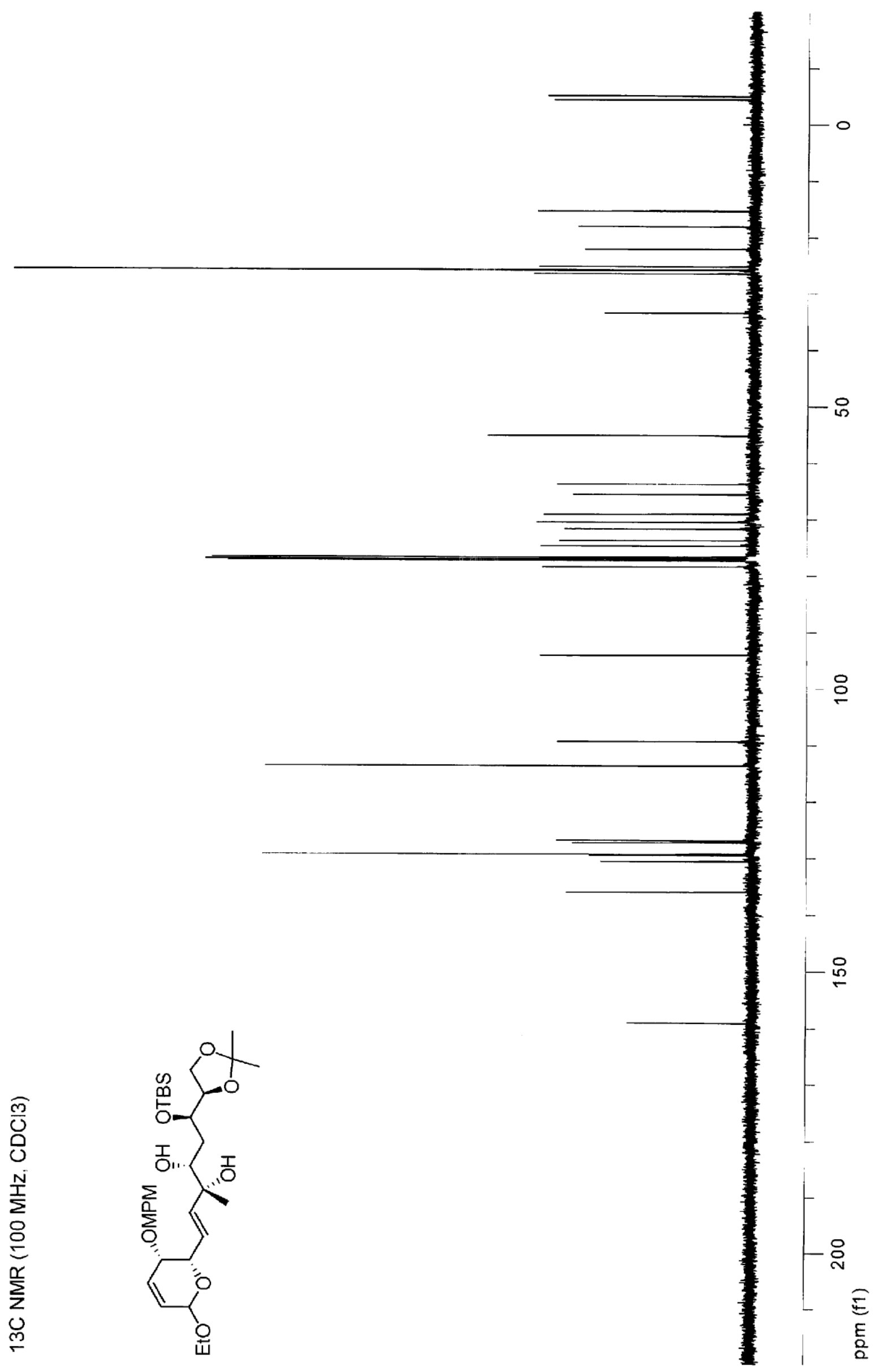




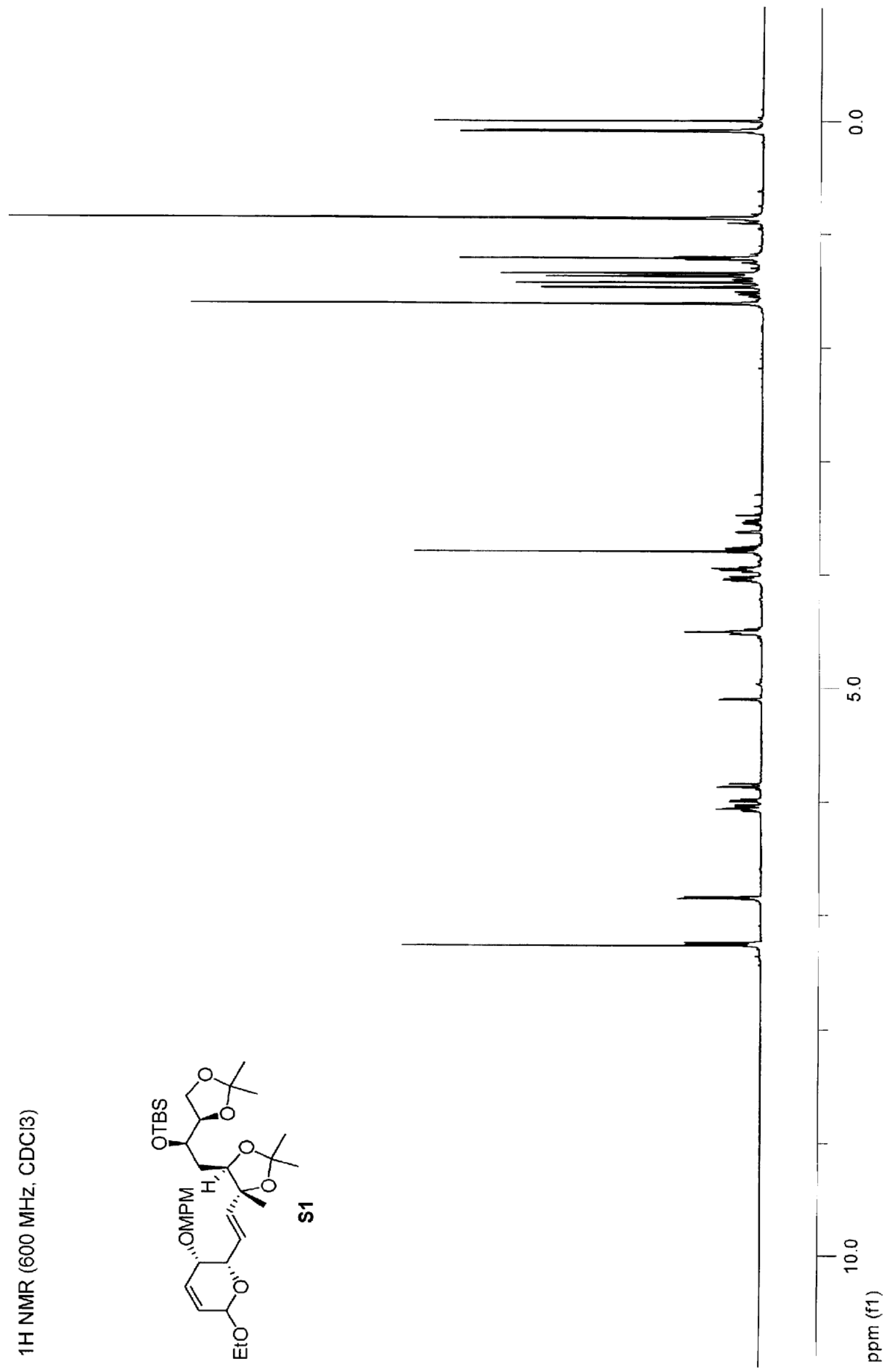




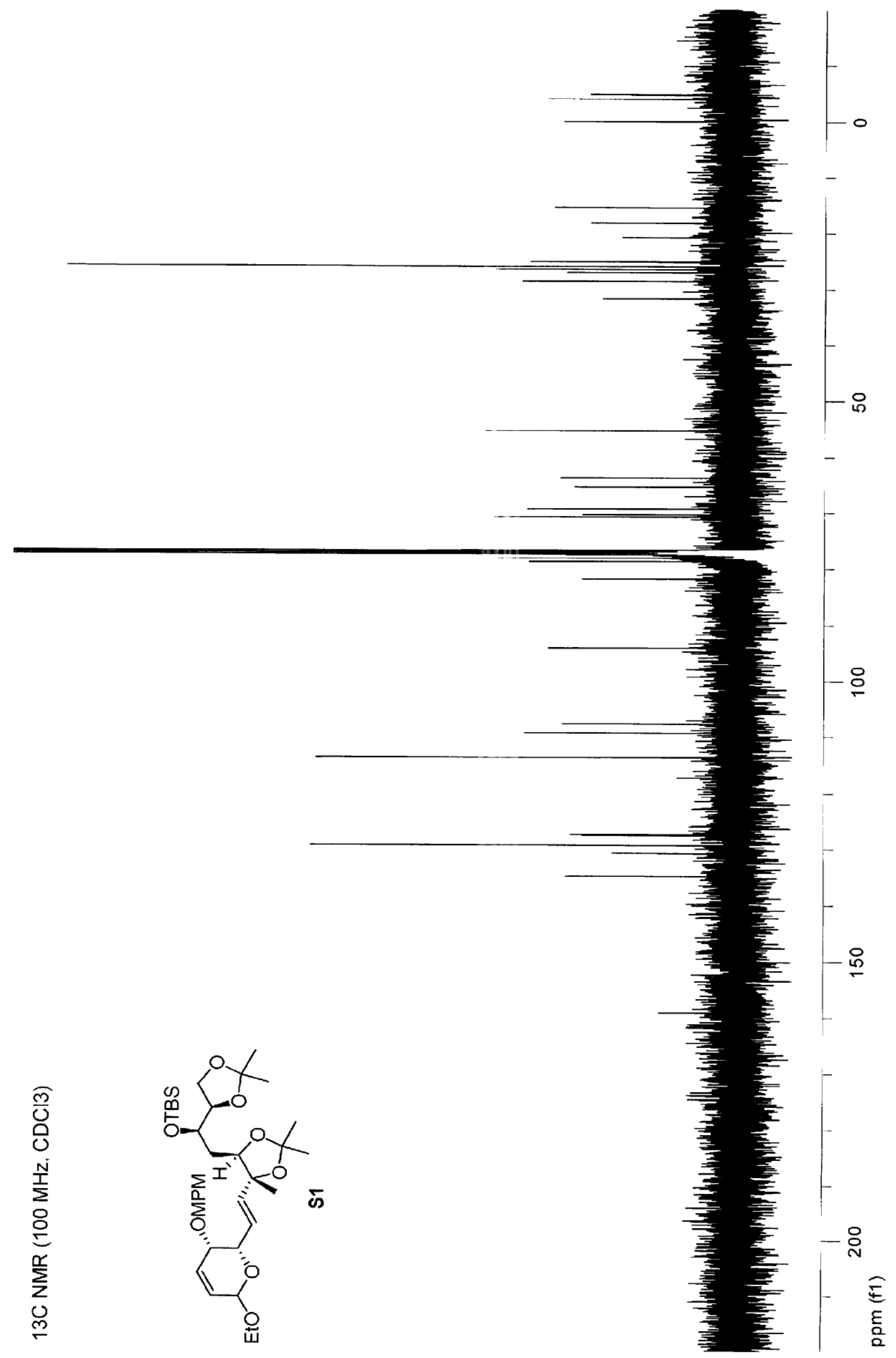




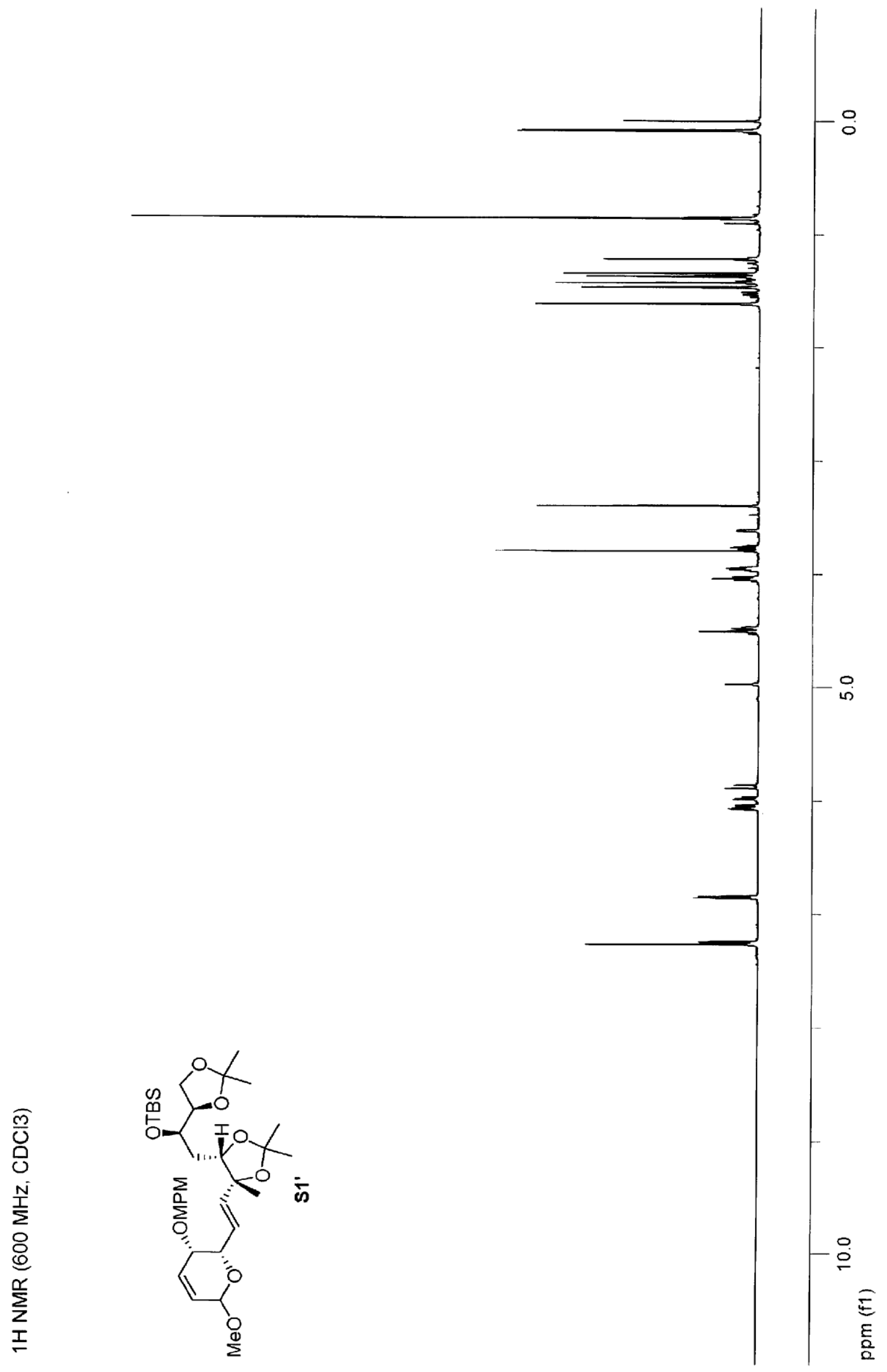




$$
1
$$




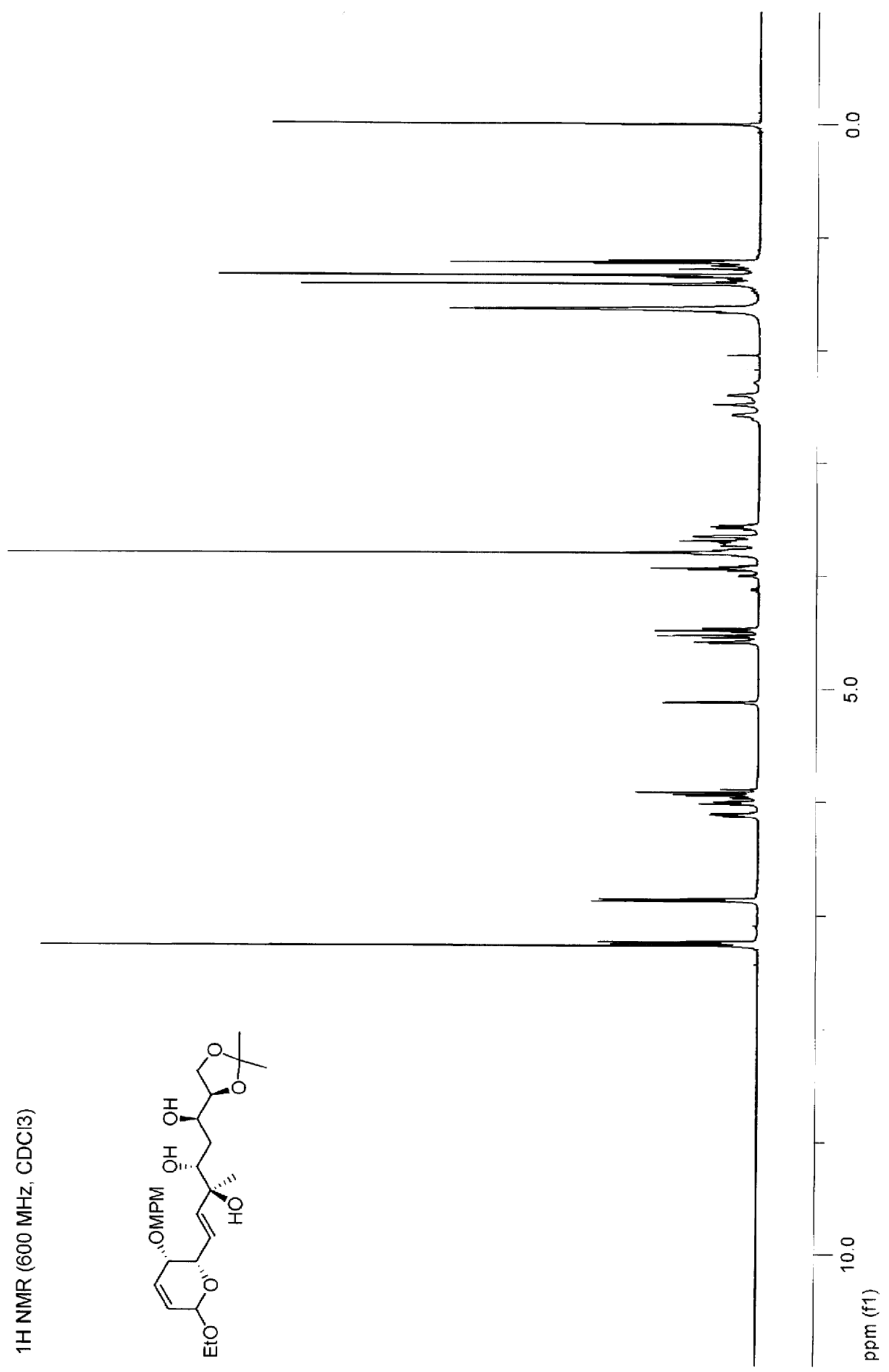




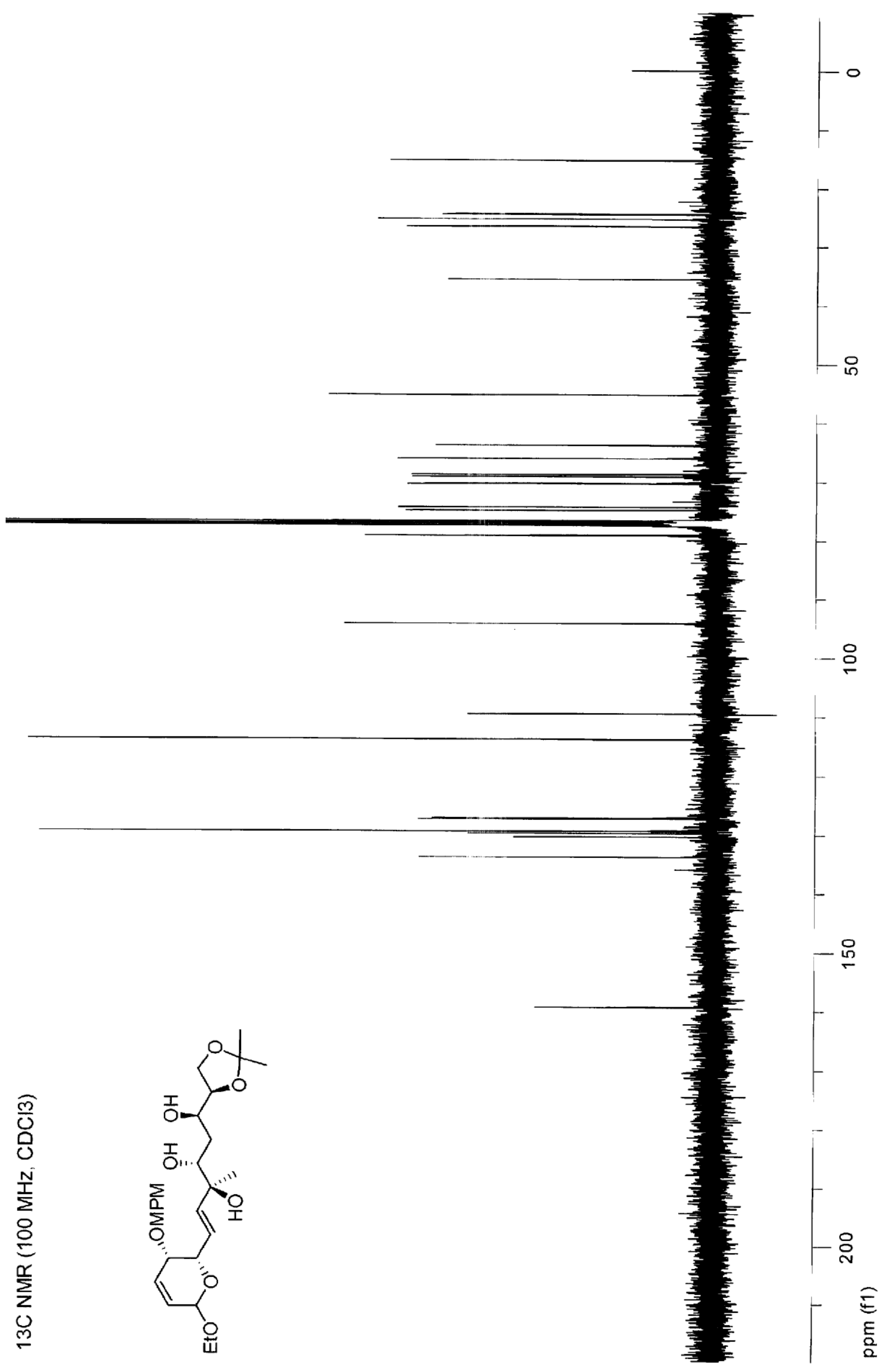




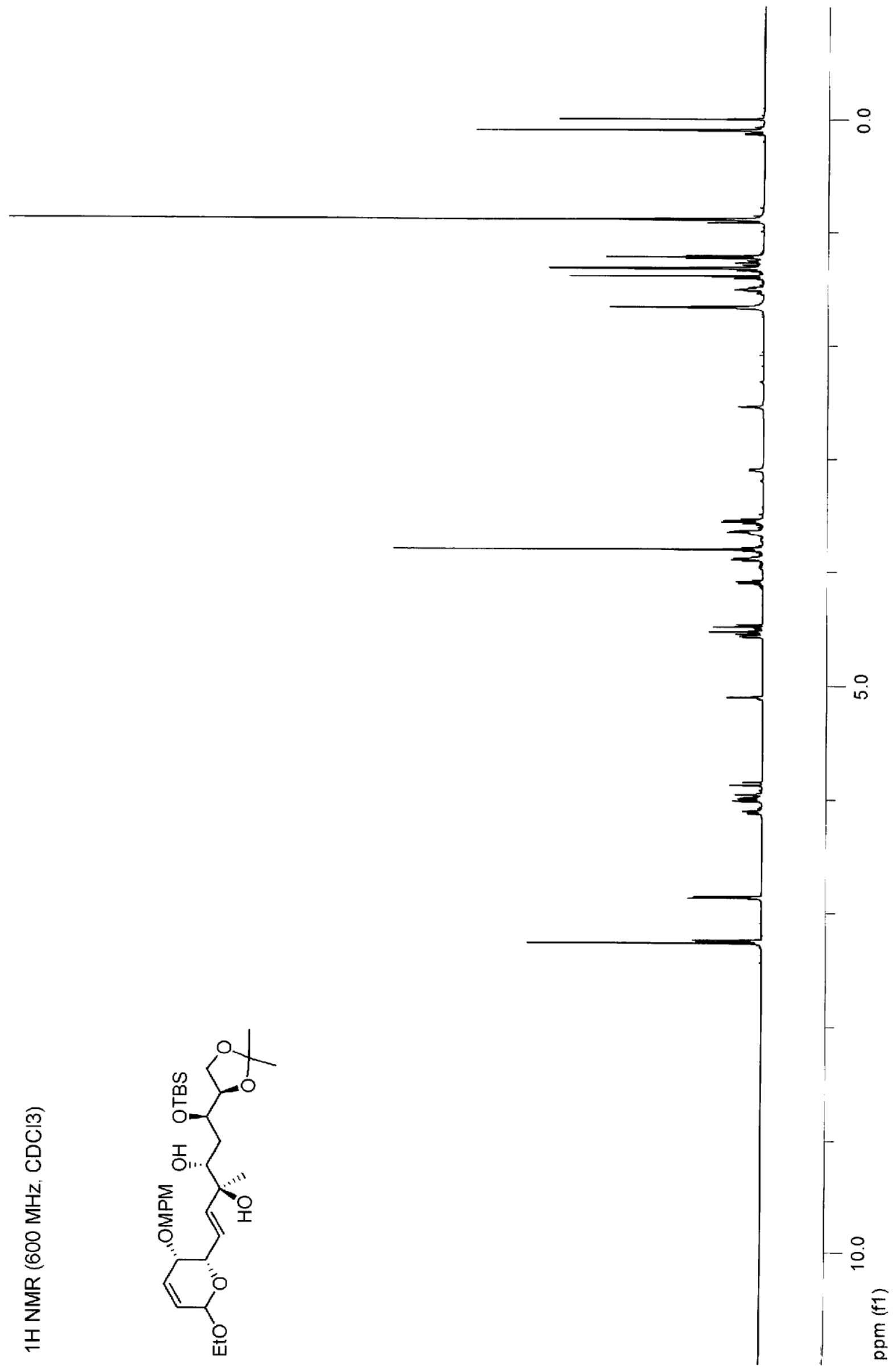




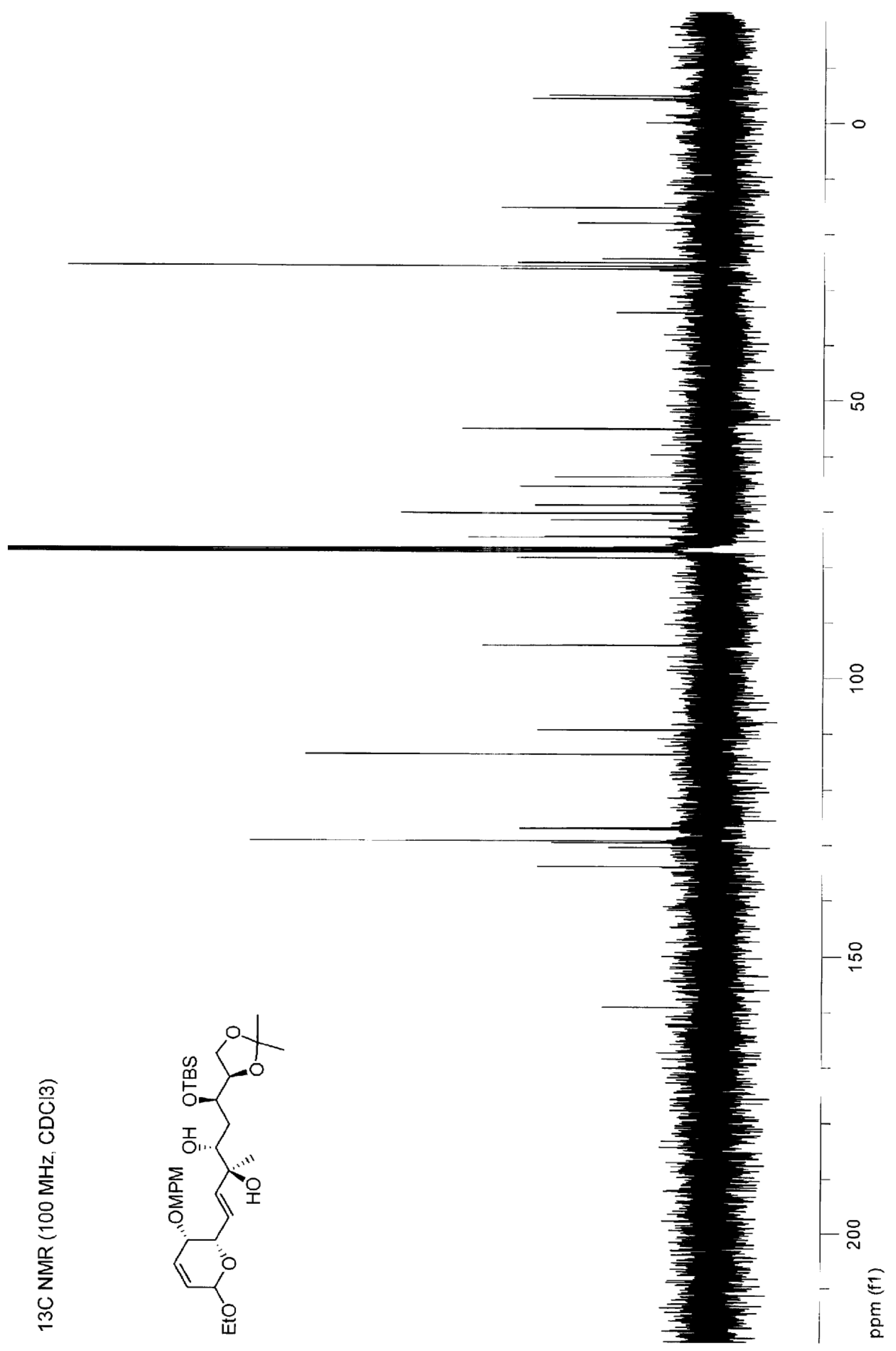

S88 


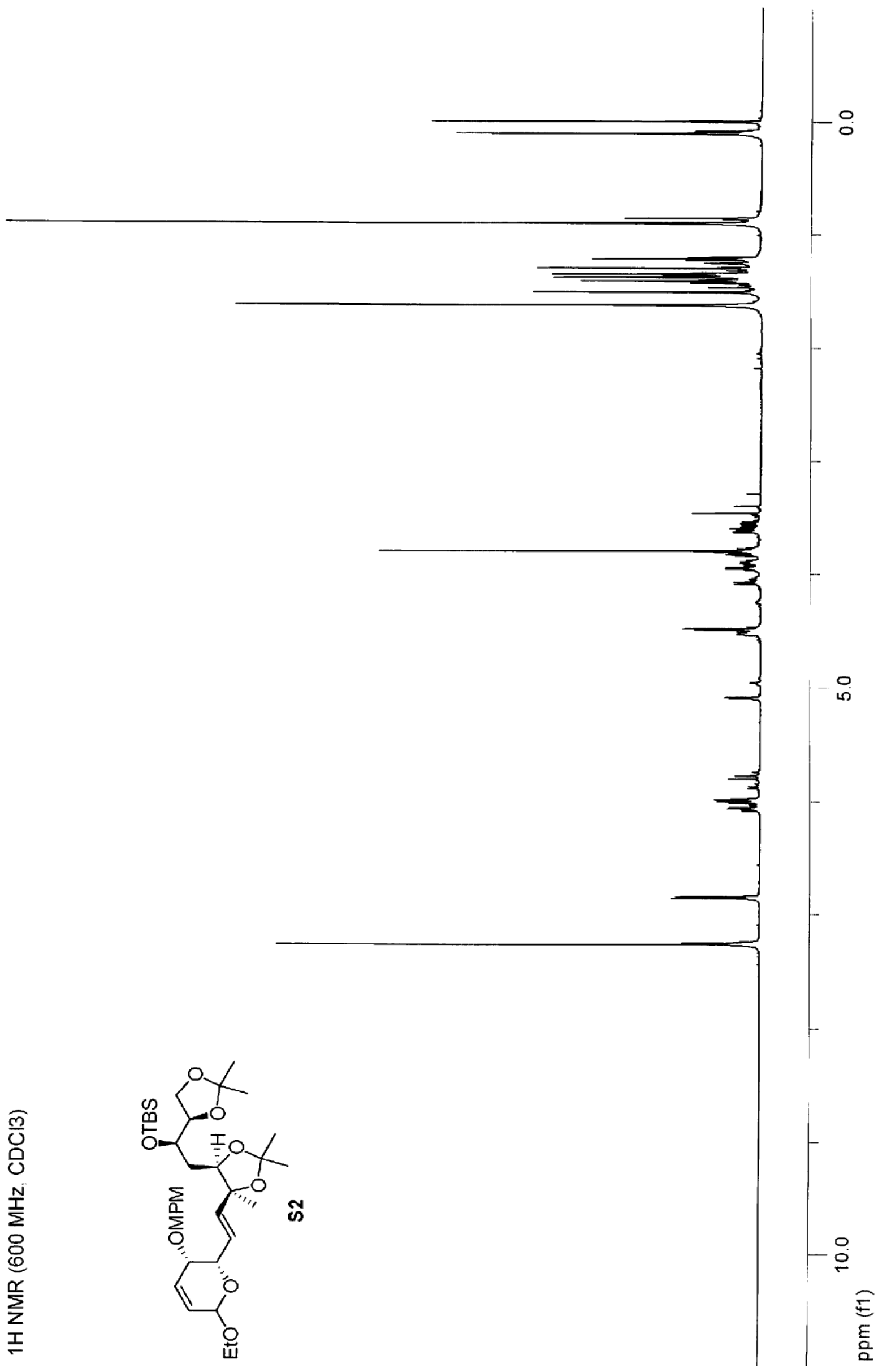




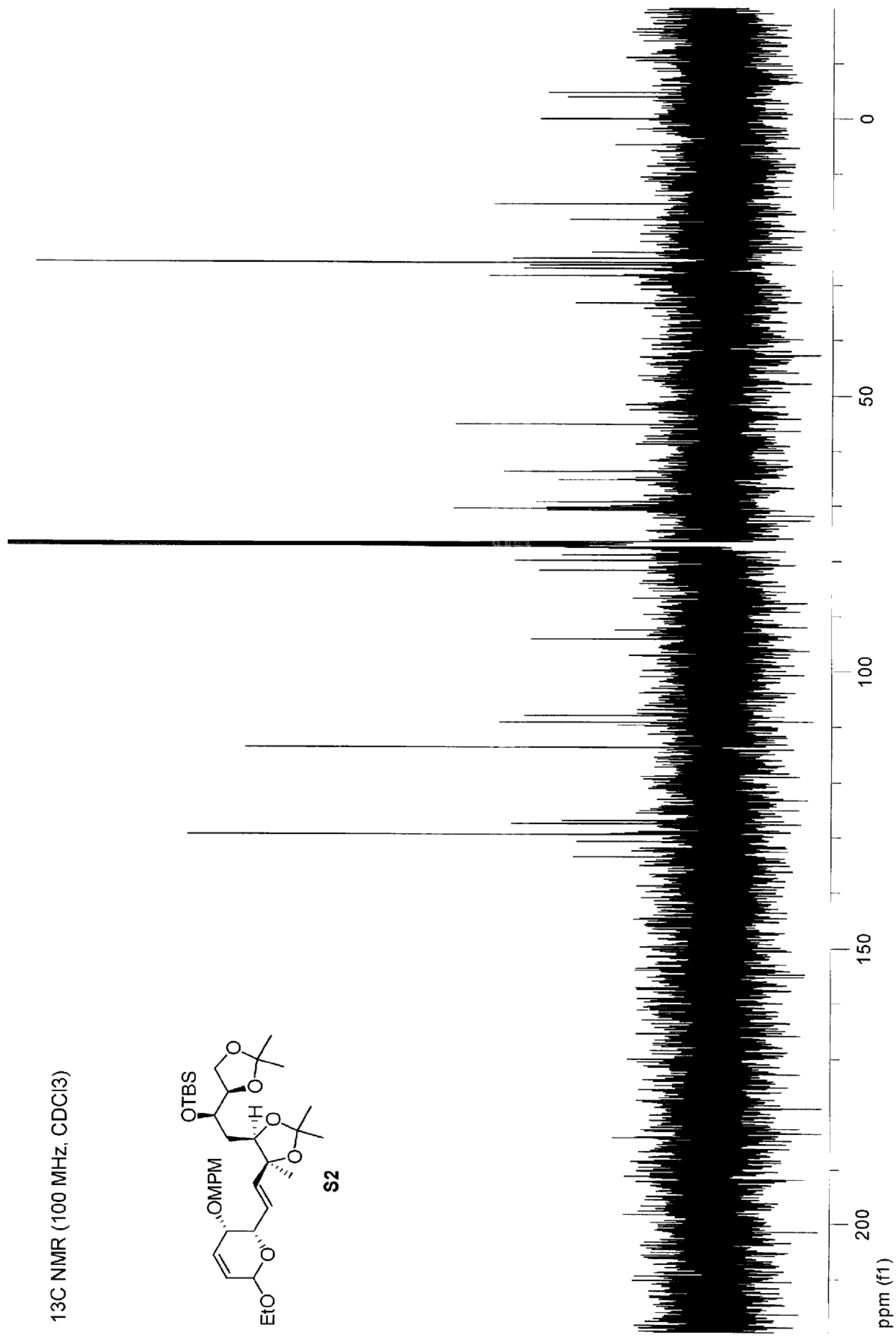




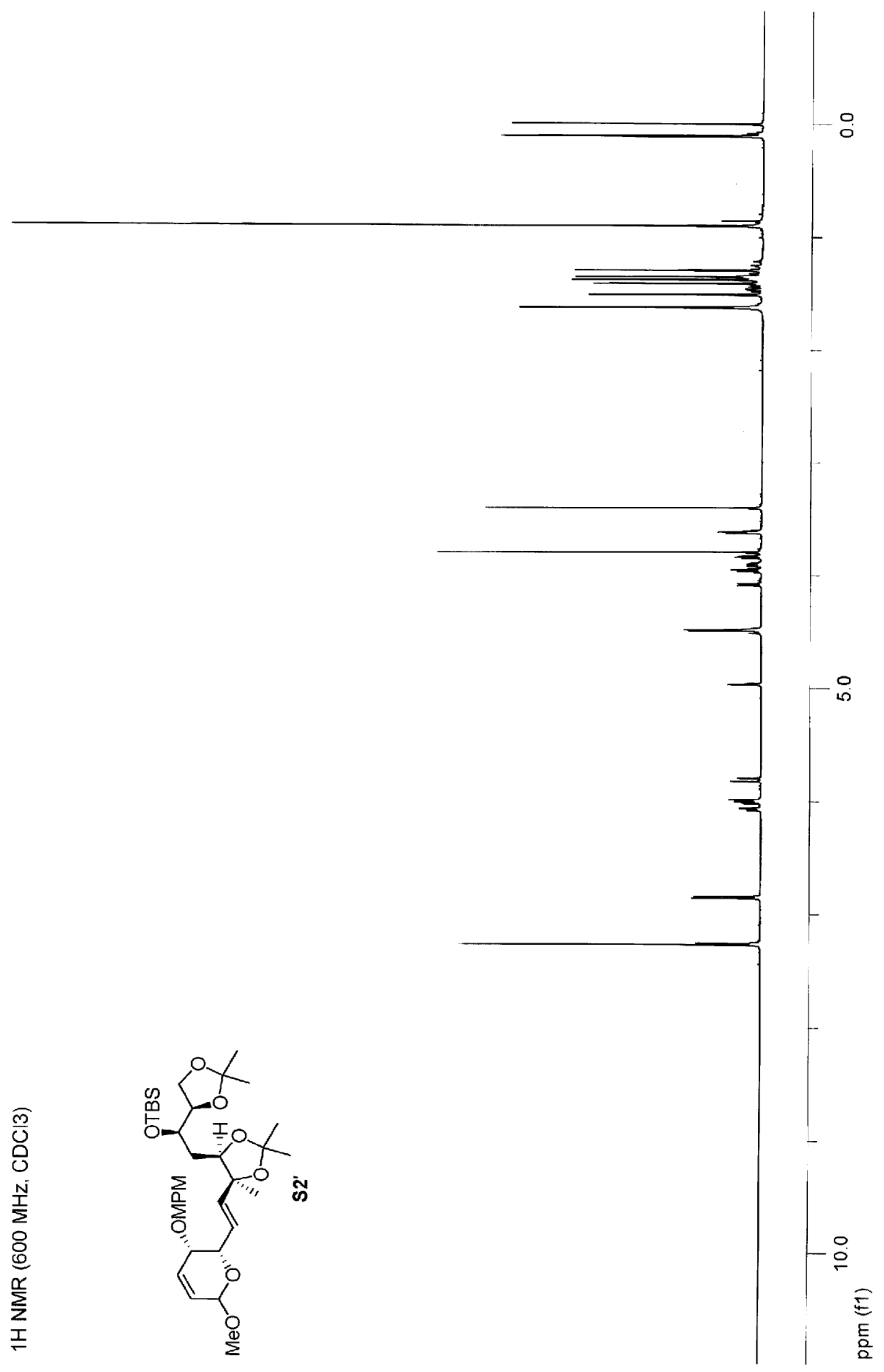




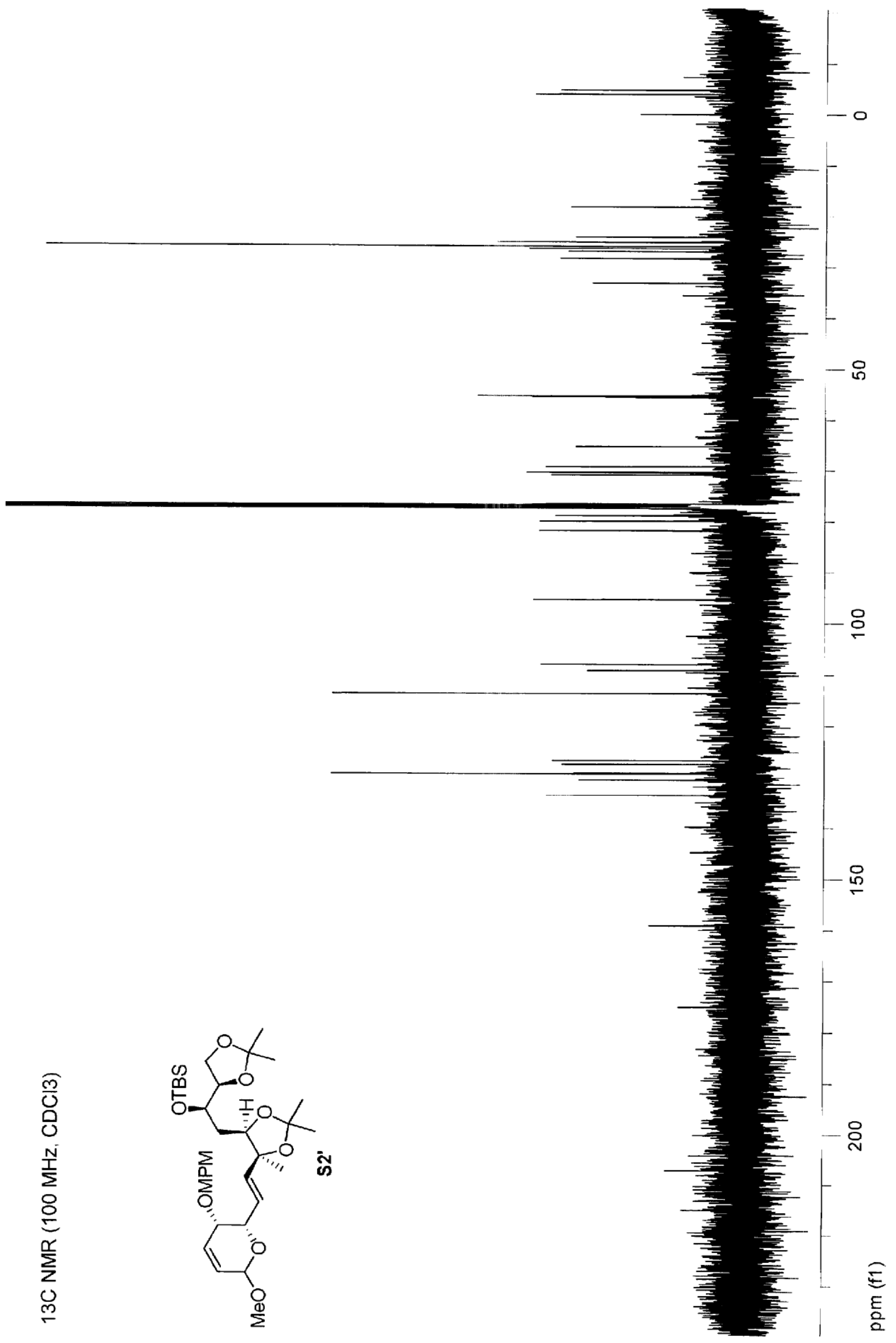

